Table 15.1

Cancer of the Lung and Bronchus (Invasive)

Trends in SEER Incidence ab and U.S. Mortality ${ }^{\mathrm{c}}$ Using the Joinpoint Regression Program, 1975-2007 With up to Four Joinpoints, 1992-2007 With up to Two Joinpoints, Both Sexes by Race/Ethnicity

\begin{tabular}{|c|c|c|c|c|c|c|c|c|c|c|c|c|}
\hline & \multicolumn{2}{|c|}{ Joinpoint Trend 1} & \multicolumn{2}{|c|}{ Joinpoint Trend 2} & \multicolumn{2}{|c|}{ Joinpoint Trend 3} & \multicolumn{2}{|c|}{$\underline{\text { Joinpoint Trend } 4}$} & \multicolumn{2}{|c|}{ Joinpoint Trend 5} & \multicolumn{2}{|c|}{$\operatorname{AAPC}^{\mathrm{d}}$} \\
\hline & Years & $\mathrm{APC}$ & Years & APC & Years & $\overline{A P C}$ & Years & $\mathrm{APC}$ & Years & $\overline{A P C}$ & $1998-2007$ & $2003-2007$ \\
\hline \multicolumn{13}{|c|}{ SEER 9 Delay-Adjusted Incidence ${ }^{a}, 1975-2007$} \\
\hline All Races & $1975-1982$ & $2.5 *$ & $1982-1991$ & $1.0 *$ & $1991-2007$ & $-0.8^{*}$ & & & & & $-0.8 *$ & $-0.8^{*}$ \\
\hline White & $1975-1982$ & $2.5 *$ & $1982-1991$ & $1.1 *$ & $1991-2007$ & $-0.6 *$ & & & & & $-0.6^{*}$ & $-0.6 *$ \\
\hline Black & $1975-1985$ & $3.3^{*}$ & $1985-2007$ & $-1.0 *$ & & & & & & & $-1.0 *$ & $-1.0 *$ \\
\hline \multicolumn{13}{|c|}{ SEER 13 Delay-Adjusted Incidence ${ }^{b}, 1992-2007$} \\
\hline All Races & $1992-2007$ & $-1.1 *$ & & & & & & & & & $-1.1 *$ & $-1 \cdot 1 *$ \\
\hline White & $1992-2007$ & $-1.0 *$ & & & & & & & & & $-1.0 *$ & $-1.0 *$ \\
\hline Black & $1992-2007$ & $-1.4 *$ & & & & & & & & & $-1.4 *$ & $-1.4 *$ \\
\hline \multicolumn{13}{|c|}{ SEER 9 Observed Incidence ${ }^{a}, 1975-2007$} \\
\hline All Races & $1975-1982$ & $2.5 *$ & $1982-1991$ & $1.0 *$ & $1991-2007$ & $-0.8 *$ & & & & & $-0.8 *$ & $-0.8 *$ \\
\hline White & $1975-1987$ & $2.0 *$ & $1987-1998$ & -0.1 & $1998-2007$ & $-1 \cdot 1 *$ & & & & & $-1 \cdot 1 *$ & $-1 \cdot 1 *$ \\
\hline Black & $1975-1984$ & $3.3 *$ & $1984-1998$ & -0.5 & $1998-2007$ & $-2.0 *$ & & & & & $-2.0 *$ & $-2.0 *$ \\
\hline \multicolumn{13}{|c|}{ SEER 13 Observed Incidence ${ }^{\mathrm{b}}, 1992-2007$} \\
\hline All Races & $1992-2007$ & $-1.2^{\star}$ & & & & & & & & & $-1.2^{\star}$ & $-1.2^{\star}$ \\
\hline White & $1992-1998$ & $-0.5^{*}$ & $1998-2007$ & -1.4 * & & & & & & & $-1.4 *$ & $-1.4^{*}$ \\
\hline White $\mathrm{NH}^{\mathrm{ef}}$ & $1992-1998$ & -0.4 & $1998-2007$ & $-1.2 *$ & & & & & & & $-1.2 *$ & $-1 \cdot 2 *$ \\
\hline Black & $1992-2007$ & $-1.5 *$ & & & & & & & & & $-1.5 *$ & $-1.5 *$ \\
\hline Black $\mathrm{NH}^{\mathrm{ef}}$ & $1992-2007$ & $-1.5^{*}$ & & & & & & & & & $-1.5 *$ & $-1.5 *$ \\
\hline $\mathrm{API}^{\mathrm{e}}$ & $1992-1994$ & -4.3 & $1994-2005$ & -0.2 & $2005-2007$ & -4.5 & & & & & -1.2 & -2.4 \\
\hline $\mathrm{AI} / \mathrm{AN}^{\mathrm{eg}}$ & $1992-2007$ & -0.5 & & & & & & & & & -0.5 & -0.5 \\
\hline Hispanic ${ }^{f}$ & $1992-2007$ & $-1 \cdot 7 \star$ & & & & & & & & & $-1 \cdot 7 \star$ & $-1 \cdot 7 \star$ \\
\hline \multicolumn{13}{|c|}{ U.S. Cancer Mortalityc, 1975-2007 } \\
\hline All Races & $1975-1980$ & $3.0 *$ & $1980-1990$ & $1.8 *$ & $1990-1994$ & -0.1 & $1994-2003$ & $-0.9 *$ & $2003-2007$ & $-1 \cdot 6^{*}$ & $-1 \cdot 2^{\star}$ & $-1 \cdot 6^{*}$ \\
\hline White & $1975-1982$ & $2.6^{*}$ & 1982-1991 & $1.7 *$ & $1991-2002$ & $-0.6 *$ & $2002-2007$ & $-1 \cdot 4$ * & & & $-1 \cdot 1 *$ & $-1.4 *$ \\
\hline Black & $1975-1981$ & $3.5 *$ & $1981-1990$ & $1.9 *$ & $1990-1997$ & $-0.9 *$ & $1997-2007$ & $-1.9 *$ & & & $-1.9 *$ & $-1.9 *$ \\
\hline \multicolumn{13}{|c|}{ U.S. Cancer Mortalityc, 1992-2007 } \\
\hline All Races & $1992-2002$ & $-0.8^{\star}$ & $2002-2007$ & $-1.5 *$ & & & & & & & $-1.2 \star$ & $-1.5 *$ \\
\hline White & $1992-2002$ & $-0.6^{*}$ & $2002-2007$ & $-1.4 *$ & & & & & & & $-1.1 *$ & $-1 \cdot 4 *$ \\
\hline White $\mathrm{NH}^{\mathrm{ef}}$ & $1992-2003$ & $-0.5^{\star}$ & $2003-2007$ & $-1.4^{*}$ & & & & & & & $-0.9 *$ & $-1.4^{*}$ \\
\hline Black & 1992-1997 & $-1.1 *$ & $1997-2005$ & $-1.7 \star$ & $2005-2007$ & $-2 \cdot 6^{*}$ & & & & & $-1.9 *$ & $-2.2^{\star}$ \\
\hline Black $\mathrm{NH}^{\mathrm{ef}}$ & $1992-2004$ & $-1.4 *$ & $2004-2007$ & -2.2 * & & & & & & & $-1.7 \star$ & $-2 \cdot 0 *$ \\
\hline $\mathrm{API}^{\mathrm{e}}$ & $1992-2007$ & $-1.3 *$ & & & & & & & & & $-1 \cdot 3 *$ & $-1 \cdot 3 *$ \\
\hline $\mathrm{AI} / \mathrm{AN}^{\mathrm{eg}}$ & $1992-2007$ & -0.2 & & & & & & & & & -0.2 & -0.2 \\
\hline Hispanic ${ }^{f}$ & 1992-1997 & 0.4 & $1997-2007$ & $-2.2 *$ & & & & & & & $-2.2 \star$ & $-2.2 *$ \\
\hline
\end{tabular}

Joinpoint Regression Program Version 3.4.3, April 2010, National Cancer Institute. (http://srab.cancer.gov/joinpoint/).

The APC is the Annual Percent Change based on rates age-adjusted to the 2000 US Std Population (19 age groups - Census P25-1130). Trends are from the SEER 9 areas (San Francisco, Connecticut, Detroit, Hawail, Iowa, New Mexico, Seattle, Utah, and Atlanta). Trends are from the SEER 13 areas (SEER 9 Areas, Los Angeles, San Jose-Monterey, Rural Georgia, and the Alaska Native Registry). Trends are from US Mortality Files, National Center for Health Statistics, Centers for Disease Control and Prevention. The AAPC is the Average Annual Percent Change and is based on the APCs calculated by Joinpoint. API - Asian/Pacific Islander, AI/AN - American Indian/Alaska Native, NH - Non-Hispanic

Hispanic and Non-Hispanic are not mutually exclusive from whites, blacks, Asian/Pacific Islanders, and American Indians/Alaska Natives. Incidence data for Hispanics and Non-Hispanics are based on NHIA and exclude cases from the Alaska Native Registry. The Hispanic and Non-Hispanic mortality trends exclude deaths from Cr, DC, ME, MD, MN, NH, NY, ND, OK and VT. Data for American Indian/Alaska Native are based on the CHSDA(Contract Health Service Delivery Area) counties.

The APC/AAPC is significantly different from zero ( $\mathrm{p}<.05)$.

Joinpoint regression line analysis could not be performed on data series. 
Table 15.2

Cancer of the Lung and Bronchus (Invasive)

Trends in SEER Incidence ab and U.S. Mortality ${ }^{c}$ Using the Joinpoint Regression Program, 1975-2007 With up to Four Joinpoints, 1992-2007 With up to Two Joinpoints,

Males by Race/Ethnicity

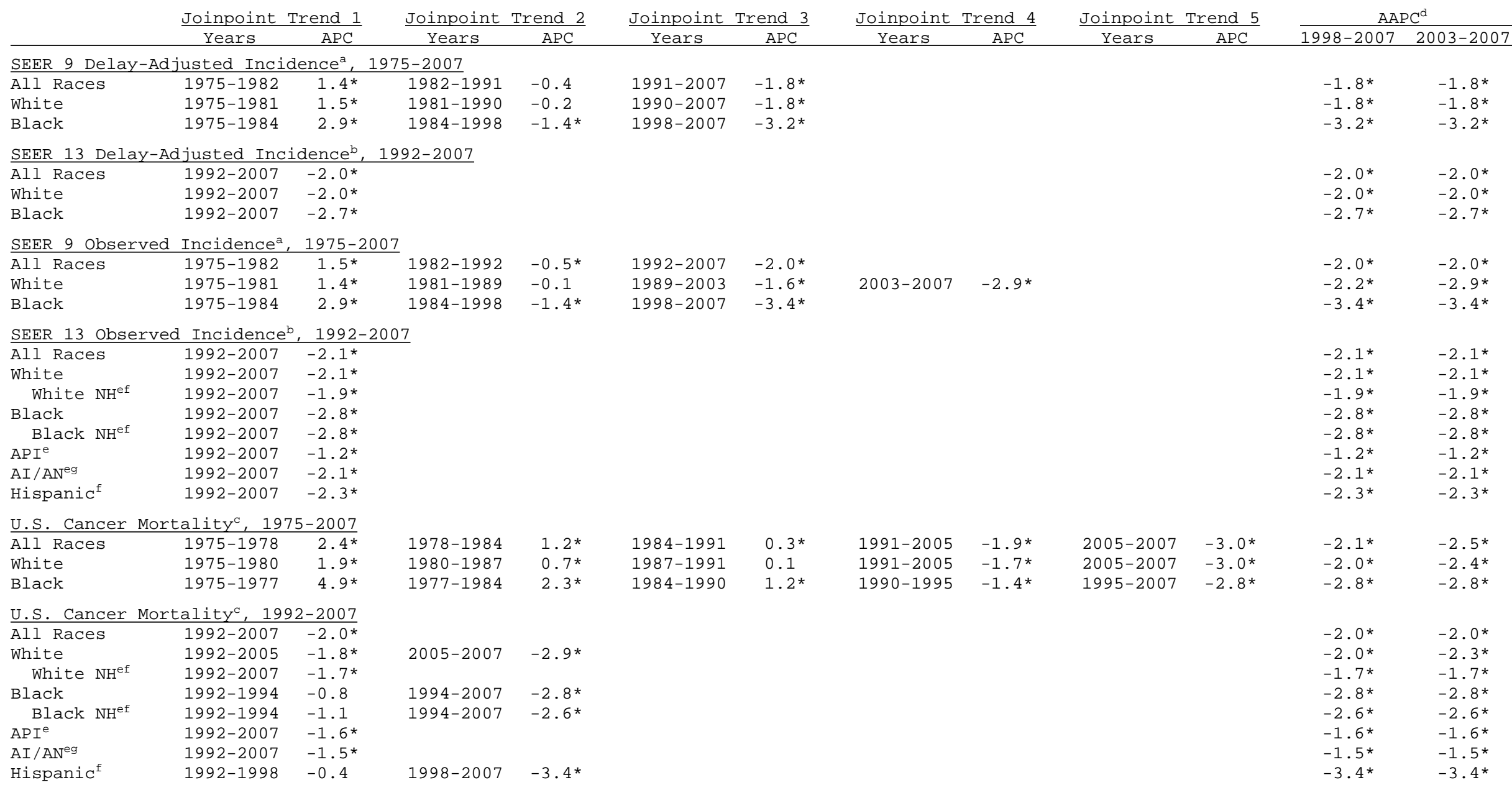

Joinpoint Regression Program Version 3.4.3, April 2010, National Cancer Institute. (http://srab.cancer.gov/joinpoint/).

The APC is the Annual Percent Change based on rates age-adjusted to the 2000 US Std Population (19 age groups - Census P25-1130).

Trends are from the SEER 9 areas (San Francisco, Connecticut, Detroit, Hawail, Iowa, New Mexico, Seattle, Utah, and Atlanta).

Trends are from the SEER 13 areas (SEER 9 Areas, Los Angeles, San Jose-Monterey, Rural Georgia, and the Alaska Native Registry).

Trends are from US Mortality Files, National Center for Health Statistics, Centers for Disease Control and Prevention.

The AAPC is the Average Annual Percent Change and is based on the APCs calculated by Joinpoint.

API - Asian/Pacific Islander, AI/AN - American Indian/Alaska Native, NH - Non-Hispanic

Hispanic and Non-Hispanic are not mutually exclusive from whites, blacks, Asian/Pacific Islanders, and American Indians/Alaska

Natives. Incidence data for Hispanics and Non-Hispanics are based on NHIA and exclude cases from the Alaska Native Registry.

The Hispanic and Non-Hispanic mortality trends exclude deaths from Cr, DC, ME, MD, MN, NH, NY, ND, OK and VT.

Data for American Indian/Alaska Native are based on the CHSDA(Contract Health Service Delivery Area) counties.

The APC/AAPC is significantly different from zero (p<.05).

Joinpoint regression line analysis could not be performed on data series. 
Table 15.3

Cancer of the Lung and Bronchus (Invasive)

Trends in SEER Incidence ab and U.S. Mortality ${ }^{c}$ Using the Joinpoint Regression Program, 1975-2007 With up to Four Joinpoints, 1992-2007 With up to Two Joinpoints, Females by Race/Ethnicity

\begin{tabular}{|c|c|c|c|c|c|c|c|c|c|c|c|c|}
\hline & Joinpoint & rend 1 & Joinpoint & rend 2 & Joinpoint & end 3 & Joinpoint & rend 4 & Joinpoint & rend 5 & & \\
\hline & Years & $\overline{\mathrm{APC}}$ & Years & $\overline{A P C}$ & Years & $\overline{\mathrm{APC}}$ & Years & APC & Years & $\overline{\mathrm{APC}}$ & $1998-2007$ & $2003-200$ \\
\hline SEER 9 Delay & justed Inci & $\mathrm{nce}^{\mathrm{a}}$, & $5-2007$ & & & & & & & & & \\
\hline All Races & $1975-1982$ & $5.5^{*}$ & $1982-1991$ & $3.5 *$ & $1991-2007$ & $0.4 *$ & & & & & 0.4 * & 0.4 * \\
\hline White & $1975-1982$ & $5.6^{*}$ & $1982-1988$ & $4.1 *$ & 1988-1997 & $1.5 *$ & $1997-2007$ & 0.2 & & & 0.2 & 0.2 \\
\hline Black & $1975-1990$ & $4.3 *$ & $1990-2007$ & 0.4 & & & & & & & 0.4 & 0.4 \\
\hline SEER 13 Dela & djusted Inc & dence $e^{b}$, & $92-2007$ & & & & & & & & & \\
\hline All Races & 1992-1997 & 0.7 & $1997-2007$ & $-0.3 *$ & & & & & & & $-0.3^{*}$ & $-0.3 *$ \\
\hline White & 1992-1997 & 0.9 & $1997-2007$ & $-0.3 *$ & & & & & & & $-0.3 *$ & $-0.3 *$ \\
\hline Black & $1992-2007$ & 0.5 & & & & & & & & & $0.5 *$ & $0.5 *$ \\
\hline SEER 9 Obser & Incidence ${ }^{a}$ & $1975-2$ & & & & & & & & & & \\
\hline All Races & $1975-1982$ & $5.5 *$ & $1982-1990$ & $3.5 *$ & $1990-1998$ & $1.0 *$ & $1998-2007$ & -0.1 & & & -0.1 & -0.1 \\
\hline White & $1975-1982$ & $5.6 *$ & $1982-1988$ & $4.1 *$ & 1988-1997 & $1.6 *$ & $1997-2007$ & 0.0 & & & 0.0 & 0.0 \\
\hline Black & $1975-1990$ & $4.3 *$ & $1990-2007$ & 0.3 & & & & & & & 0.3 & 0.3 \\
\hline SEER 13 Obse & Incidence & $1992-$ & & & & & & & & & & \\
\hline All Races & $1992-1998$ & 0.6 & -1998-2007 & $-0.6^{*}$ & & & & & & & $-0.6^{*}$ & $-0.6^{*}$ \\
\hline White & 1992-1997 & $1.0 *$ & $1997-2007$ & $-0.5^{\star}$ & & & & & & & $-0.5 *$ & $-0.5 *$ \\
\hline White $\mathrm{NH}^{\mathrm{ef}}$ & 1992-1997 & $1.2^{*}$ & $1997-2007$ & -0.3 & & & & & & & -0.3 & -0.3 \\
\hline Black & $1992-2007$ & 0.3 & & & & & & & & & 0.3 & 0.3 \\
\hline Black $\mathrm{NH}^{\mathrm{ef}}$ & $1992-2007$ & 0.4 & & & & & & & & & 0.4 & 0.4 \\
\hline $\mathrm{API}^{\mathrm{e}}$ & $1992-2007$ & 0.2 & & & & & & & & & 0.2 & 0.2 \\
\hline $\mathrm{AI} / \mathrm{AN}^{\mathrm{eg}}$ & $1992-2007$ & 1.7 & & & & & & & & & 1.7 & 1.7 \\
\hline Hispanic ${ }^{f}$ & $1992-2007$ & $-1.0 *$ & & & & & & & & & $-1.0 *$ & $-1.0 *$ \\
\hline U.S. Cancer & Eality ${ }^{c}, 19$ & -2007 & & & & & & & & & & \\
\hline All Races & $1975-1982$ & $6.0^{*}$ & $1982-1990$ & $4.2 *$ & $1990-1995$ & $1.7 \star$ & $1995-2003$ & $0.3 *$ & $2003-2007$ & $-0.9 *$ & -0.2 & $-0.9 *$ \\
\hline White & $1975-1983$ & $5.8 *$ & 1983-1990 & $4.1 *$ & 1990-1994 & $2.1 *$ & $1994-2002$ & $0.5 *$ & $2002-2007$ & $-0.6 *$ & -0.1 & $-0.6 *$ \\
\hline Black & $1975-1981$ & $6.7^{\star}$ & $1981-1991$ & $3.9 *$ & 1991-1998 & $1.0 *$ & $1998-2007$ & -0.3 & & & -0.3 & -0.3 \\
\hline U.S. Cancer & Eality ${ }^{c}, 19$ & -2007 & & & & & & & & & & \\
\hline All Races & $1992-2002$ & $0.6^{\star}$ & $2002-2007$ & $-0.8 *$ & & & & & & & -0.2 & $-0.8 *$ \\
\hline White & $1992-2002$ & $0.7 *$ & $2002-2007$ & $-0.7 *$ & & & & & & & -0.1 & $-0.7 *$ \\
\hline White $\mathrm{NH}^{\mathrm{ef}}$ & $1992-2002$ & $0.9 *$ & $2002-2007$ & -0.5 & & & & & & & 0.1 & -0.5 \\
\hline Black & $1992-1998$ & $1.1 *$ & $1998-2007$ & -0.3 & & & & & & & -0.3 & -0.3 \\
\hline Black $\mathrm{NH}^{\mathrm{ef}}$ & $1992-2005$ & $0.6^{*}$ & $2005-2007$ & -2.6 & & & & & & & -0.1 & -1.0 \\
\hline $\mathrm{API} \mathrm{I}^{\mathrm{e}}$ & $1992-2007$ & -0.4 & & & & & & & & & -0.4 & -0.4 \\
\hline $\mathrm{AI} / \mathrm{AN}^{\mathrm{eg}}$ & $1992-2007$ & $1.5 *$ & & & & & & & & & $1.5 *$ & $1.5 *$ \\
\hline Hispanic ${ }^{f}$ & $1992-2007$ & -0.3 & & & & & & & & & -0.3 & -0.3 \\
\hline
\end{tabular}

Joinpoint Regression Program Version 3.4.3, April 2010, National Cancer Institute. (http://srab.cancer.gov/joinpoint/).

The APC is the Annual Percent Change based on rates age-adjusted to the 2000 US Std Population (19 age groups - Census P25-1130). Trends are from the SEER 9 areas (San Francisco, Connecticut, Detroit, Hawail, Iowa, New Mexico, Seattle, Utah, and Atlanta). Trends are from the SEER 13 areas (SEER 9 Areas, LOS Angeles, San Jose-Monterey, Rural Georgia, and the Alaska Native Registry). Trends are from US Mortality Files, National Center for Health Statistics, Centers for Disease Control and Prevention. The AAPC is the Average Annual Percent Change and is based on the APCs calculated by Joinpoint. API - Asian/Pacific Islander, AI/AN - American Indian/Alaska Native, NH - Non-Hispanic

Hispanic and Non-Hispanic are not mutually exclusive from whites, blacks, Asian/Pacific Islanders, and American Indians/Alaska Natives. Incidence data for Hispanics and Non-Hispanics are based on NHIA and exclude cases from the Alaska Native Registry. The Hispanic and Non-Hispanic mortality trends exclude deaths from CI, DC, ME, MD, MN, NH, NY, ND, OK and VT. Data for American Indian/Alaska Native are based on the CHSDA(Contract Health Service Delivery Area) counties.

The APC/AAPC is significantly different from zero ( $\mathrm{p}<.05)$.

Joinpoint regression line analysis could not be performed on data series. 
Table 15.4

Small Cell Cancer of the Lung and Bronchus (Invasive) and Non-Small Cell Cancer of the Lung and Bronchus (Invasive)

Trends in SEER 9 Observed Incidence ${ }^{a}$ Using the Joinpoint Regression Program,

1975-2007 With up to Four Joinpoints,

By Race and Sex

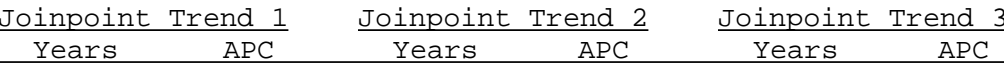

$\begin{array}{cccc}\text { Joinpoint } & \text { Trend } 4 & \text { Joinpoint } & \text { Trend } 5 \\ \text { Years } & \text { APC } & \text { Years } & \text { APC }\end{array}$

$\frac{\mathrm{AAPC}}{2003-2007}$

SEER 9 Observed Incidence ${ }^{a}$ for Small Cell Cancer of the Lung and Bronchus, 1975-2007

$\begin{array}{llrrrrrr}\text { All Races } & \text { Both Sexes } & 1975-1982 & 6.2^{*} & 1982-1989 & 1.2^{*} & 1989-2007 & -2.3^{*} \\ \text { All Races } & \text { Male } & 1975-1981 & 5.5^{*} & 1981-1988 & 0.1 & 1988-2007 & -3^{*} \\ \text { All Races } & \text { Female } & 1975-1982 & 9.6^{*} & 1982-1991 & 2.9 * & 1991-2007 & -1.7^{*} \\ \text { White } & \text { Both Sexes } & 1975-1982 & 6.3^{*} & 1982-1989 & 1.4^{*} & 1989-2007 & -2.2^{*} \\ \text { White } & \text { Male } & 1975-1980 & 6.3^{*} & 1980-1988 & 0.5 & 1988-2007 & -3.2^{*} \\ \text { White } & \text { Female } & 1975-1982 & 10.0^{*} & 1982-1990 & 3.5^{*} & 1990-2007 & -1.3^{*} \\ & & & & & \\ \text { Black } & \text { Both Sexes } & 1975-1986 & 4.8^{*} & 1986-2007 & -2.4^{*} & & \\ \text { Black } & \text { Male } & 1975-1985 & 4.9^{*} & 1985-2007 & -3.4^{*} & \\ \text { Black } & \text { Female } & 1975-1991 & 4.6^{*} & 1991-2007 & -2.0^{*} & \end{array}$

$-2 \cdot 3 *$

$-3.2$

$-1.7 *$

$-2.2 *$

-3.2 *

$-1.3 *$

-2.4 *

-3.4 *

$-2.0 *$

SEER 9 Observed Incidence ${ }^{a}$ for Non-Small Cell Cancer of the Lung and Bronchus, 1975-2007

$\begin{array}{lllllllc}\text { All Races } & \text { Both Sexes } & 1975-1988 & 1.5^{*} & 1988-1998 & 0.0 & 1998-2007 & -1.0^{*} \\ \text { All Races } & \text { Male } & 1975-1987 & 0.5^{*} & 1987-2003 & -1.3^{*} & 2003-2007 & -3.2^{*} \\ \text { All Races } & \text { Female } & 1975-1988 & 4.3^{*} & 1988-1997 & 1.7^{*} & 1997-2007 & 0.3^{2} \\ & & & & & & & \\ \text { White } & \text { Both Sexes } & 1975-1988 & 1.6^{*} & 1988-1998 & 0.1 & 1998-2007 & -0.9^{*} \\ \text { White } & \text { Male } & 1975-1987 & 0.4^{*} & 1987-2003 & -1.3^{*} & 2003-2007 & -3.1^{*} \\ \text { White } & \text { Female } & 1975-1988 & 4.4^{*} & 1988-1997 & 1.9 * & 1997-2007 & 0.3^{*} \\ & & & & & & & \\ \text { Black } & \text { Both Sexes } & 1975-1984 & 3.0^{*} & 1984-1998 & -0.3 & 1998-2007 & -1.8^{*} \\ \text { Black } & \text { Male } & 1975-1984 & 2.6^{*} & 1984-1998 & -1.2^{*} & 1998-2007 & -3.3^{*} \\ \text { Black } & \text { Female } & 1975-1990 & 4.2^{*} & 1990-2007 & 0.6^{*} & & \end{array}$

$-1.0 *$

$-3.2 *$

0.3

-0.9 *

$-1.8 *$

$-3.3 *$

$0.6^{*}$

Joinpoint Regression Program Version 3.4.3, April 2010, National Cancer Institute. (http://srab.cancer.gov/joinpoint/).

The APC is the Annual Percent Change based on rates age-adjusted to the 2000 US Std Population (19 age groups - Census P25-1130). Trends are from the SEER 9 areas (San Francisco, Connecticut, Detroit, Hawail, Iowa, New Mexico, Seattle, Utah, and Atlanta). The AAPC is the Average Annual Percent Change and is based on the APCs calculated by Joinpoint.

Joinpoint regression line analysis could not be performed on data series. 
Table 15.5

Cancer of the Lung and Bronchus (Invasive)

Delay-adjusted SEER Incidence ${ }^{a}$ Rates by Year, Race and Sex

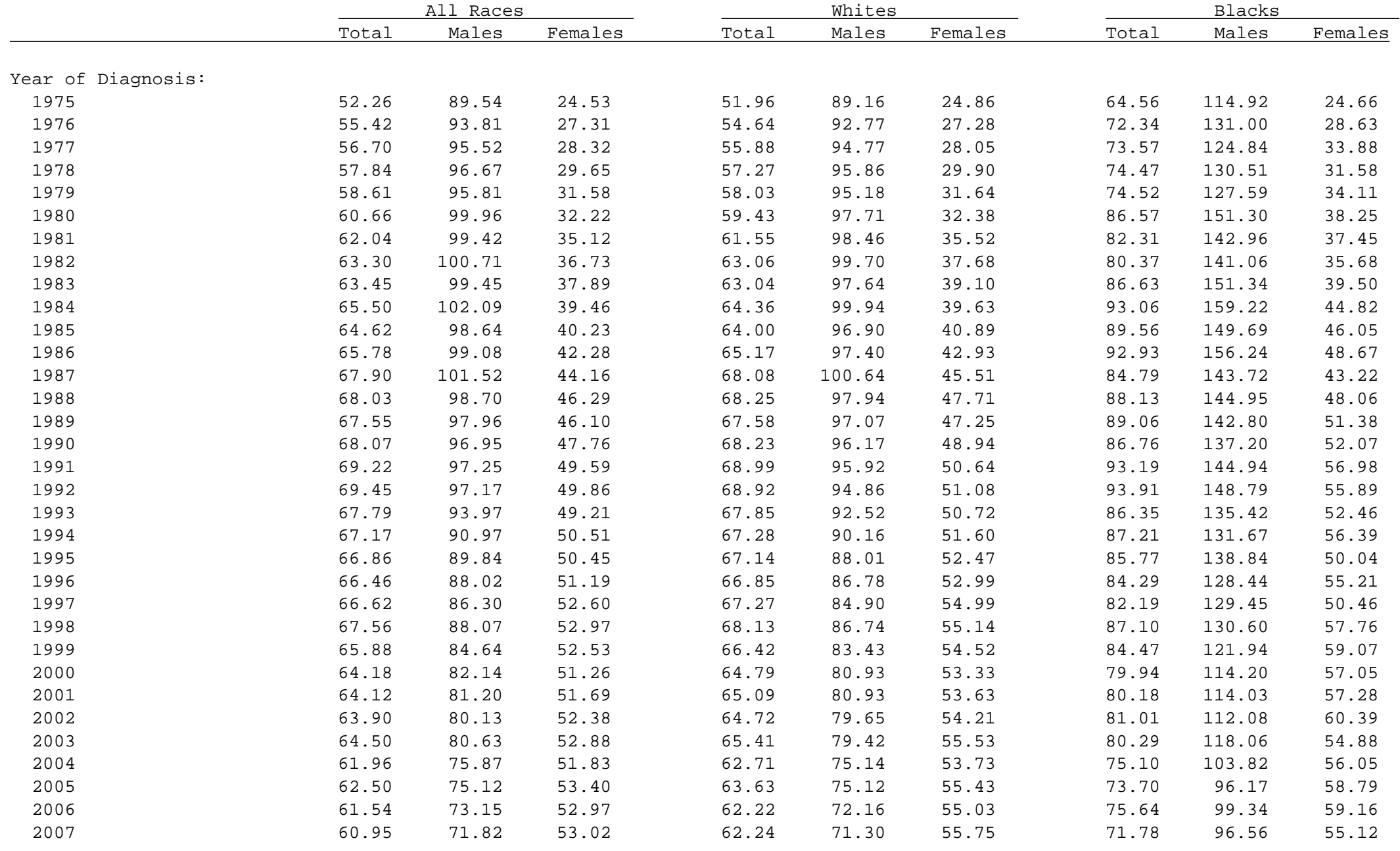

Additional information on the model used to delay-adjust SEER Incidence rates can be found at (http://srab.cancer.gov/delay/). SEER 9 areas (San Francisco, Connecticut, Detroit, Hawail, Iowa, New Mexico, Seattle, Utah, and Atlanta).

Rates are per 100,000 and are age-adjusted to the 2000 Us std Population (19 age groups - Census P25-113 
Table 15.6

Cancer of the Lung and Bronchus (Invasive)

Age-adjusted SEER Incidence ${ }^{a}$ Rates by Year, Race and Sex

\begin{tabular}{|c|c|c|c|c|c|c|c|c|c|}
\hline & \multicolumn{3}{|c|}{ All Races } & \multicolumn{3}{|c|}{ Whites } & \multicolumn{3}{|c|}{ Blacks } \\
\hline & Total & Males & Females & Total & Males & Females & Total & Males & Females \\
\hline \multicolumn{10}{|c|}{ Year of Diagnosis: } \\
\hline 1975 & 52.26 & 89.54 & 24.53 & 51.96 & 89.16 & 24.86 & 64.56 & 114.92 & 24.66 \\
\hline 1976 & 55.42 & 93.81 & 27.31 & 54.64 & 92.77 & 27.28 & 72.34 & 131.00 & 28.63 \\
\hline 1977 & 56.70 & 95.52 & 28.32 & 55.88 & 94.77 & 28.05 & 73.57 & 124.84 & 33.88 \\
\hline 1978 & 57.84 & 96.67 & 29.65 & 57.27 & 95.86 & 29.90 & 74.47 & 130.51 & 31.58 \\
\hline 1979 & 58.61 & 95.81 & 31.58 & 58.03 & 95.18 & 31.64 & 74.52 & 127.59 & 34.11 \\
\hline 1980 & 60.66 & 99.96 & 32.22 & 59.43 & 97.71 & 32.38 & 86.57 & 151.30 & 38.25 \\
\hline 1981 & 62.04 & 99.42 & 35.12 & 61.55 & 98.46 & 35.52 & 82.31 & 142.96 & 37.45 \\
\hline 1982 & 63.30 & 100.71 & 36.73 & 63.06 & 99.70 & 37.68 & 80.37 & 141.06 & 35.68 \\
\hline 1983 & 63.45 & 99.45 & 37.89 & 63.04 & 97.64 & 39.10 & 86.62 & 151.34 & 39.49 \\
\hline 1984 & 65.50 & 102.08 & 39.46 & 64.35 & 99.94 & 39.63 & 93.06 & 159.21 & 44.82 \\
\hline 1985 & 64.62 & 98.64 & 40.23 & 64.00 & 96.90 & 40.89 & 89.56 & 149.68 & 46.04 \\
\hline 1986 & 65.78 & 99.08 & 42.28 & 65.16 & 97.39 & 42.93 & 92.92 & 156.23 & 48.67 \\
\hline 1987 & 67.90 & 101.51 & 44.16 & 68.07 & 100.63 & 45.50 & 84.78 & 143.70 & 43.22 \\
\hline 1988 & 68.02 & 98.69 & 46.29 & 68.24 & 97.92 & 47.70 & 88.12 & 144.93 & 48.06 \\
\hline 1989 & 67.54 & 97.95 & 46.10 & 67.57 & 97.06 & 47.24 & 89.04 & 142.77 & 51.37 \\
\hline 1990 & 68.06 & 96.94 & 47.75 & 68.22 & 96.15 & 48.92 & 86.74 & 137.17 & 52.06 \\
\hline 1991 & 69.21 & 97.24 & 49.58 & 68.97 & 95.89 & 50.63 & 93.15 & 144.90 & 56.96 \\
\hline 1992 & 69.43 & 97.15 & 49.85 & 68.90 & 94.83 & 51.06 & 93.87 & 148.73 & 55.86 \\
\hline 1993 & 67.77 & 93.94 & 49.19 & 67.82 & 92.48 & 50.69 & 86.30 & 135.35 & 52.43 \\
\hline 1994 & 67.14 & 90.93 & 50.49 & 67.24 & 90.11 & 51.57 & 87.15 & 131.58 & 56.36 \\
\hline 1995 & 66.83 & 89.79 & 50.42 & 67.09 & 87.94 & 52.43 & 85.70 & 138.72 & 50.00 \\
\hline 1996 & 66.42 & 87.97 & 51.16 & 66.79 & 86.70 & 52.94 & 84.20 & 128.30 & 55.15 \\
\hline 1997 & 66.57 & 86.23 & 52.56 & 67.19 & 84.80 & 54.92 & 82.08 & 129.27 & 50.39 \\
\hline 1998 & 67.50 & 87.99 & 52.93 & 68.03 & 86.62 & 55.07 & 86.96 & 130.39 & 57.67 \\
\hline 1999 & 65.78 & 84.52 & 52.45 & 66.27 & 83.25 & 54.40 & 84.27 & 121.64 & 58.92 \\
\hline 2000 & 64.06 & 81.98 & 51.17 & 64.62 & 80.70 & 53.19 & 79.70 & 113.85 & 56.88 \\
\hline 2001 & 63.96 & 81.00 & 51.57 & 64.86 & 80.65 & 53.44 & 79.87 & 113.59 & 57.06 \\
\hline 2002 & 63.69 & 79.87 & 52.21 & 64.42 & 79.29 & 53.97 & 80.60 & 111.52 & 60.09 \\
\hline 2003 & 64.22 & 80.28 & 52.65 & 65.02 & 78.95 & 55.20 & 79.78 & 117.30 & 54.53 \\
\hline 2004 & 61.60 & 75.43 & 51.54 & 62.23 & 74.57 & 53.32 & 74.48 & 102.97 & 55.59 \\
\hline 2005 & 62.02 & 74.54 & 52.99 & 63.01 & 74.38 & 54.89 & 72.92 & 95.15 & 58.17 \\
\hline 2006 & 60.91 & 72.40 & 52.43 & 61.43 & 71.24 & 54.33 & 74.61 & 97.98 & 58.35 \\
\hline 2007 & 59.34 & 69.93 & 51.62 & 60.35 & 69.13 & 54.06 & 69.47 & 93.45 & 53.35 \\
\hline $1975-2007$ & 63.99 & 89.57 & 45.39 & 64.05 & 88.67 & 46.39 & 81.77 & 127.33 & 49.80 \\
\hline
\end{tabular}

SEER 9 areas (San Francisco, Connecticut, Detroit, Hawaii, Iowa, New Mexico, Seattle, Utah, and Atlanta). 
Table 15.7

Small Cell Cancer of the Lung and Bronchus (Invasive)

Age-adjusted SEER Incidence ${ }^{a}$ Rates by Year, Race and Sex

\begin{tabular}{|c|c|c|c|c|c|c|c|c|c|}
\hline & \multicolumn{3}{|c|}{ All Races } & \multicolumn{3}{|c|}{ Whites } & \multicolumn{3}{|c|}{ Blacks } \\
\hline & Total & Males & Females & Total & Males & Females & Total & Males & Females \\
\hline \multicolumn{10}{|c|}{ Year of Diagnosis: } \\
\hline 1975 & 6.65 & 10.34 & 3.79 & 6.89 & 10.70 & 3.97 & 5.87 & 8.94 & 3.48 \\
\hline 1976 & 7.06 & 11.36 & 3.84 & 7.12 & 11.52 & 3.89 & 8.23 & 12.82 & 4.76 \\
\hline 1977 & 7.70 & 12.38 & 4.22 & 7.84 & 12.79 & 4.21 & 8.13 & 11.19 & 5.78 \\
\hline 1978 & 8.64 & 13.39 & 5.03 & 8.87 & 13.69 & 5.28 & 7.81 & 13.89 & 3.13 \\
\hline 1979 & 8.68 & 13.09 & 5.36 & 9.03 & 13.63 & 5.61 & 7.63 & 11.83 & 4.47 \\
\hline 1980 & 9.10 & 14.20 & 5.23 & 9.33 & 14.40 & 5.54 & 9.99 & 17.53 & 4.38 \\
\hline 1981 & 9.71 & 14.55 & 6.12 & 10.01 & 14.84 & 6.48 & 9.21 & 15.87 & 4.21 \\
\hline 1982 & 10.37 & 14.92 & 7.12 & 10.73 & 15.28 & 7.55 & 9.63 & 15.94 & 4.92 \\
\hline 1983 & 10.36 & 14.86 & 7.08 & 10.74 & 15.04 & 7.64 & 9.12 & 15.08 & 4.85 \\
\hline 1984 & 10.69 & 15.18 & 7.41 & 11.05 & 15.64 & 7.77 & 10.41 & 14.99 & 6.86 \\
\hline 1985 & 10.44 & 14.96 & 7.08 & 10.78 & 15.25 & 7.49 & 11.07 & 17.77 & 6.27 \\
\hline 1986 & 11.20 & 15.52 & 7.98 & 11.54 & 15.99 & 8.27 & 12.26 & 17.70 & 8.35 \\
\hline 1987 & 10.65 & 14.13 & 8.19 & 11.17 & 14.56 & 8.84 & 9.53 & 14.49 & 6.03 \\
\hline 1988 & 11.37 & 15.27 & 8.51 & 11.90 & 15.61 & 9.21 & 10.49 & 17.86 & 5.30 \\
\hline 1989 & 11.24 & 14.84 & 8.68 & 11.68 & 15.22 & 9.21 & 12.07 & 17.67 & 8.35 \\
\hline 1990 & 10.65 & 13.84 & 8.38 & 11.26 & 14.46 & 9.05 & 9.35 & 13.70 & 6.21 \\
\hline 1991 & 11.29 & 13.99 & 9.36 & 11.68 & 14.32 & 9.84 & 12.34 & 15.92 & 10.00 \\
\hline 1992 & 10.49 & 13.39 & 8.44 & 10.96 & 13.84 & 8.96 & 10.16 & 13.32 & 7.95 \\
\hline 1993 & 10.02 & 12.33 & 8.34 & 10.48 & 12.64 & 8.94 & 9.38 & 13.25 & 6.74 \\
\hline 1994 & 9.77 & 11.69 & 8.42 & 10.24 & 11.98 & 9.05 & 9.95 & 13.62 & 7.43 \\
\hline 1995 & 9.85 & 12.25 & 8.13 & 10.35 & 12.60 & 8.74 & 9.50 & 13.89 & 6.61 \\
\hline 1996 & 9.70 & 12.07 & 7.96 & 10.27 & 12.46 & 8.63 & 8.80 & 12.02 & 6.84 \\
\hline 1997 & 9.53 & 10.85 & 8.64 & 10.11 & 11.11 & 9.49 & 7.86 & 10.82 & 6.05 \\
\hline 1998 & 9.28 & 10.84 & 8.14 & 9.86 & 11.27 & 8.83 & 9.37 & 12.10 & 7.45 \\
\hline 1999 & 8.80 & 10.03 & 7.97 & 9.34 & 10.25 & 8.78 & 8.14 & 10.95 & 6.05 \\
\hline 2000 & 8.78 & 10.42 & 7.58 & 9.27 & 10.84 & 8.13 & 9.44 & 11.54 & 7.93 \\
\hline 2001 & 8.34 & 9.78 & 7.33 & 8.87 & 10.26 & 7.93 & 8.50 & 10.78 & 6.86 \\
\hline 2002 & 8.25 & 9.35 & 7.50 & 8.93 & 9.81 & 8.34 & 7.20 & 9.74 & 5.70 \\
\hline 2003 & 8.17 & 9.06 & 7.56 & 8.68 & 9.32 & 8.29 & 7.88 & 9.53 & 6.72 \\
\hline 2004 & 8.02 & 8.90 & 7.40 & 8.41 & 9.13 & 7.90 & 8.54 & 9.90 & 7.68 \\
\hline 2005 & 7.50 & 8.57 & 6.75 & 8.12 & 9.12 & 7.42 & 6.47 & 7.51 & 5.80 \\
\hline 2006 & 7.56 & 8.33 & 7.01 & 8.11 & 8.63 & 7.77 & 6.79 & 8.33 & 5.75 \\
\hline 2007 & 7.40 & 8.22 & 6.82 & 8.04 & 8.55 & 7.73 & 6.64 & 8.97 & 4.98 \\
\hline $1975-2007$ & 9.26 & 11.94 & 7.27 & 9.73 & 12.35 & 7.78 & 8.89 & 12.58 & 6.29 \\
\hline
\end{tabular}


Table 15.8

Non-Small Cell Cancer of the Lung and Bronchus (Invasive)

Age-adjusted SEER Incidence ${ }^{a}$ Rates by Year, Race and Sex

\begin{tabular}{|c|c|c|c|c|c|c|c|c|c|}
\hline & \multicolumn{3}{|c|}{ All Races } & \multicolumn{3}{|c|}{ Whites } & \multicolumn{3}{|c|}{ Blacks } \\
\hline & Total & Males & Females & Total & Males & Females & Total & Males & Females \\
\hline \multicolumn{10}{|c|}{ Year of Diagnosis: } \\
\hline 1975 & 45.61 & 79.20 & 20.74 & 45.07 & 78.46 & 20.89 & 58.68 & 105.98 & 21.18 \\
\hline 1976 & 48.36 & 82.45 & 23.47 & 47.52 & 81.25 & 23.39 & 64.11 & 118.18 & 23.87 \\
\hline 1977 & 49.00 & 83.14 & 24.10 & 48.04 & 81.98 & 23.84 & 65.44 & 113.65 & 28.10 \\
\hline 1978 & 49.20 & 83.28 & 24.62 & 48.40 & 82.17 & 24.62 & 66.65 & 116.63 & 28.45 \\
\hline 1979 & 49.93 & 82.72 & 26.22 & 49.00 & 81.55 & 26.03 & 66.89 & 115.76 & 29.64 \\
\hline 1980 & 51.56 & 85.76 & 26.99 & 50.10 & 83.31 & 26.84 & 76.58 & 133.78 & 33.87 \\
\hline 1981 & 52.32 & 84.87 & 29.00 & 51.54 & 83.62 & 29.05 & 73.10 & 127.09 & 33.24 \\
\hline 1982 & 52.93 & 85.79 & 29.61 & 52.33 & 84.42 & 30.13 & 70.74 & 125.12 & 30.76 \\
\hline 1983 & 53.09 & 84.59 & 30.81 & 52.30 & 82.60 & 31.46 & 77.50 & 136.26 & 34.64 \\
\hline 1984 & 54.81 & 86.90 & 32.05 & 53.31 & 84.29 & 31.85 & 82.64 & 144.23 & 37.96 \\
\hline 1985 & 54.18 & 83.68 & 33.15 & 53.22 & 81.64 & 33.40 & 78.49 & 131.91 & 39.78 \\
\hline 1986 & 54.58 & 83.56 & 34.30 & 53.62 & 81.40 & 34.66 & 80.66 & 138.53 & 40.31 \\
\hline 1987 & 57.25 & 87.38 & 35.97 & 56.91 & 86.07 & 36.66 & 75.25 & 129.21 & 37.19 \\
\hline 1988 & 56.65 & 83.42 & 37.77 & 56.35 & 82.32 & 38.49 & 77.63 & 127.07 & 42.76 \\
\hline 1989 & 56.31 & 83.11 & 37.42 & 55.88 & 81.83 & 38.03 & 76.97 & 125.10 & 43.02 \\
\hline 1990 & 57.41 & 83.10 & 39.37 & 56.96 & 81.69 & 39.87 & 77.38 & 123.46 & 45.84 \\
\hline 1991 & 57.92 & 83.25 & 40.22 & 57.29 & 81.57 & 40.78 & 80.81 & 128.98 & 46.96 \\
\hline 1992 & 58.94 & 83.75 & 41.41 & 57.93 & 80.99 & 42.10 & 83.71 & 135.41 & 47.91 \\
\hline 1993 & 57.74 & 81.60 & 40.86 & 57.34 & 79.84 & 41.75 & 76.92 & 122.10 & 45.69 \\
\hline 1994 & 57.37 & 79.24 & 42.07 & 57.00 & 78.13 & 42.51 & 77.20 & 117.96 & 48.93 \\
\hline 1995 & 56.97 & 77.54 & 42.29 & 56.73 & 75.34 & 43.68 & 76.20 & 124.83 & 43.39 \\
\hline 1996 & 56.71 & 75.90 & 43.20 & 56.52 & 74.24 & 44.31 & 75.40 & 116.28 & 48.31 \\
\hline 1997 & 57.04 & 75.38 & 43.92 & 57.08 & 73.69 & 45.43 & 74.22 & 118.45 & 44.34 \\
\hline 1998 & 58.22 & 77.15 & 44.79 & 58.18 & 75.34 & 46.23 & 77.59 & 118.29 & 50.22 \\
\hline 1999 & 56.98 & 74.48 & 44.49 & 56.94 & 73.00 & 45.62 & 76.13 & 110.70 & 52.88 \\
\hline 2000 & 55.28 & 71.56 & 43.58 & 55.34 & 69.87 & 45.05 & 70.26 & 102.31 & 48.95 \\
\hline 2001 & 55.61 & 71.21 & 44.24 & 55.99 & 70.39 & 45.52 & 71.37 & 102.81 & 50.20 \\
\hline 2002 & 55.44 & 70.52 & 44.72 & 55.49 & 69.48 & 45.62 & 73.40 & 101.78 & 54.39 \\
\hline 2003 & 56.05 & 71.22 & 45.09 & 56.34 & 69.64 & 46.91 & 71.90 & 107.77 & 47.81 \\
\hline 2004 & 53.58 & 66.54 & 44.14 & 53.82 & 65.44 & 45.42 & 65.94 & 93.07 & 47.91 \\
\hline 2005 & 54.52 & 65.97 & 46.24 & 54.89 & 65.26 & 47.47 & 66.45 & 87.64 & 52.37 \\
\hline 2006 & 53.35 & 64.07 & 45.42 & 53.32 & 62.62 & 46.56 & 67.81 & 89.65 & 52.60 \\
\hline 2007 & 51.95 & 61.71 & 44.80 & 52.31 & 60.58 & 46.33 & 62.83 & 84.48 & 48.37 \\
\hline $1975-2007$ & 54.72 & 77.63 & 38.12 & 54.33 & 76.32 & 38.61 & 72.88 & 114.75 & 43.51 \\
\hline
\end{tabular}


Table 15.9

Cancer of the Lung and Bronchus (Invasive)

Age-adjusted U.S. Death ${ }^{a}$ Rates by Year, Race and Sex

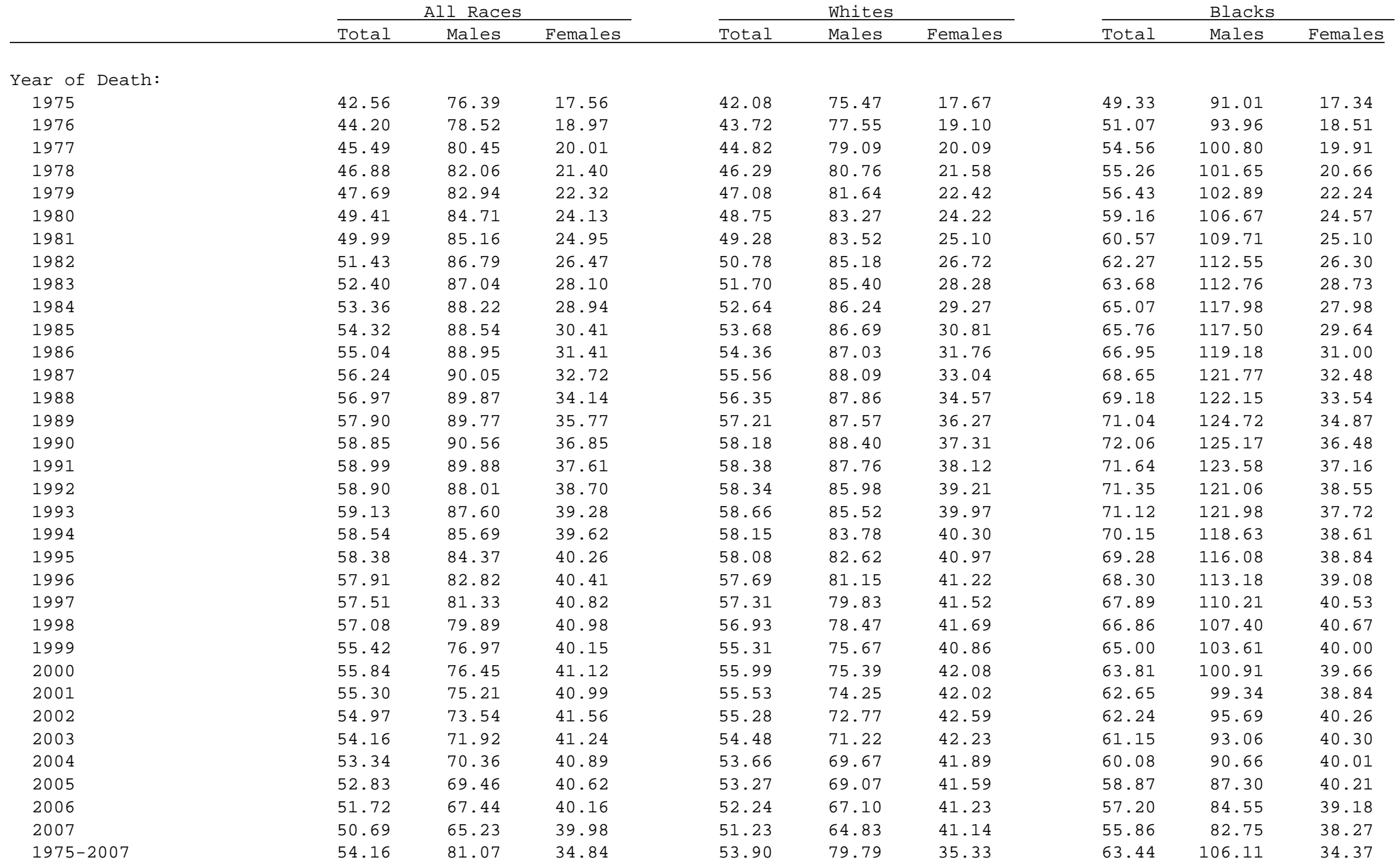

US Mortality Files, National Center for Health Statistics, Centers for Disease Control and Prevention. 


\section{SEER Incidence}

Age-Adjusted Rates, 2003-2007 All ages

65 and over

All ages (IARC world std) ${ }^{c}$

Age-Specific Rates, 2003-2007

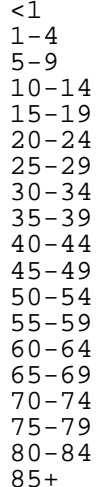

$85+$

U.S. Mortality

Age at Deat

Age-Adjusted Rates, 2003-2007 All ages

65 and over

All ages (IARC world std) ${ }^{c}$

Age-Specific Rates, 2003-2007

$<1$
$1-4$

$5-9$

$10-14$
$15-19$
$20-24$

$20-24$

$25-29$
$30-34$
$35-39$

$35-39$

$40-44$

$45-49$

$50-54$

$55-59$

$60-64$

$65-69$

$70-74$

$85-79$

$80-84$
$85+$
All Race
Total Males

Females

$\begin{array}{rrr}62.5 & 76.2 & 52.4 \\ 19.9 & 22.2 & 17.7 \\ 356.6 & 449.6 & 292.0 \\ 39.2 & 46.6 & 33.4 \\ & & \\ - & - & - \\ - & - & - \\ - & - & - \\ - & - & - \\ 0.1 & - & - \\ 0.3 & 0.3 & 0.3 \\ 0.6 & 0.6 & 0.6 \\ 1.2 & 1.1 & 1.4 \\ 3.2 & 3.1 & 3.3 \\ 9.9 & 9.6 & 10.2 \\ 25.3 & 25.3 & 25.2 \\ 49.9 & 56.0 & 44.0 \\ 93.4 & 107.4 & 80.2 \\ 172.4 & 198.5 & 148.6 \\ 275.5 & 323.3 & 233.8 \\ 371.4 & 446.4 & 310.8 \\ 436.0 & 552.2 & 352.2 \\ 415.6 & 552.4 & 329.5 \\ 299.6 & 438.2 & 233.7\end{array}$

Total

Whites

Females

Total

Blacks Females

$\begin{array}{rrr}52.5 & 68.8 & 40.6 \\ 15.9 & 19.1 & 12.9 \\ 305.7 & 412.3 & 232.1 \\ 32.2 & 41.1 & 25.2 \\ & & \\ - & - & - \\ - & - & - \\ - & - & - \\ - & - & - \\ 0.0 & 0.0 & - \\ 0.1 & 0.1 & 0.1 \\ 0.2 & 0.2 & 0.2 \\ 0.5 & 0.6 & 0.5 \\ 2.1 & 2.1 & 2.1 \\ 7.9 & 8.2 & 7.5 \\ 20.2 & 22.6 & 18.0 \\ 39.8 & 48.2 & 31.8 \\ 74.7 & 92.0 & 58.4 \\ 139.8 & 172.2 & 110.2 \\ 220.9 & 277.8 & 171.2 \\ 304.3 & 390.8 & 234.1 \\ 369.5 & 495.2 & 278.3 \\ 379.4 & 536.8 & 281.7 \\ 300.4 & 465.9 & 224.7\end{array}$

$\begin{array}{rrr}63.8 & 76.3 & 54.7 \\ 19.7 & 21.4 & 18.1 \\ 368.3 & 455.5 & 307.6 \\ 40.0 & 46.3 & 34.9 \\ & & \\ - & - & - \\ - & - & - \\ - & - & - \\ - & - & - \\ 0.1 & - & - \\ 0.3 & 0.3 & 0.4 \\ 0.6 & 0.5 & 0.6 \\ 1.1 & 1.0 & 1.3 \\ 3.1 & 2.9 & 3.3 \\ 9.5 & 9.2 & 9.9 \\ 24.3 & 23.7 & 25.0 \\ 47.8 & 52.3 & 43.4 \\ 92.5 & 102.4 & 82.9 \\ 175.7 & 197.1 & 155.6 \\ 286.1 & 326.8 & 249.9 \\ 385.4 & 451.8 & 330.6 \\ 452.2 & 561.8 & 371.9 \\ 426.5 & 560.9 & 341.9 \\ 361.9 & 441.0 & 236.6\end{array}$

426.5
301.9

441.0

Total

(1)

224.7

$\begin{array}{rr}52.9 & 68.3 \\ 15.6 & 18.3 \\ 311.0 & 413.7 \\ 32.4 & 40.6 \\ & \\ - & - \\ - & - \\ - & - \\ - & - \\ 0.0 & - \\ 0.1 & 0.1 \\ 0.2 & 0.2 \\ 0.5 & 0.6 \\ 2.1 & 2.1 \\ 7.7 & 7.9 \\ 19.3 & 21.0 \\ 38.2 & 45.2 \\ 73.4 & 88.0 \\ 140.1 & 168.5 \\ 224.1 & 276.8 \\ 310.7 & 392.4 \\ 376.5 & 497.7 \\ 384.9 & 539.4 \\ 304.5 & 469.3\end{array}$

\subsection{0 \\ 13.6
239.3}

25.8

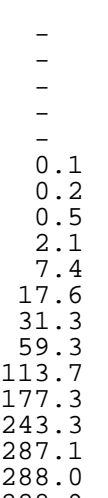

$\begin{array}{rrr}73.5 & 101.2 & 54.8 \\ 29.5 & 37.7 & 22.8 \\ 378.1 & 540.2 & 276.6 \\ 48.5 & 65.4 & 36.2\end{array}$

$\begin{array}{rrr}- & - & - \\ - & - & - \\ - & - & - \\ - & - & - \\ - & - & - \\ - & - & - \\ 0.7 & - & - \\ 1.6 & 1.7 & 1.5 \\ 3.5 & 3.6 & 3.5 \\ 15.1 & 14.8 & 15.3 \\ 42.3 & 47.0 & 38.2 \\ 83.8 & 104.6 & 66.1 \\ 140.6 & 193.9 & 96.5 \\ 230.4 & 305.9 & 170.5 \\ 321.7 & 441.5 & 231.0 \\ 396.8 & 550.0 & 289.0 \\ 441.7 & 623.5 & 328.9 \\ 418.3 & 620.2 & 307.2 \\ 307.6 & 501.2 & 226.0\end{array}$

SEER 17 areas.

Files, National Center for Health Statistics, Centers for Disease Control and Prevention.

Rates are per 100,000 and are age-adjusted to the 2000 US Std Population (19 age groups - Census P25-1130), unless noted.

Rates are per 100,000 and are age-adjusted to the IARC world standard population.
Statistic not shown. Rate based on less than 16 cases for the time interval. 
SEER Incidence ${ }^{c}$ Rates, Age-Adjusted and Age-Specific Rates, by Race and Sex

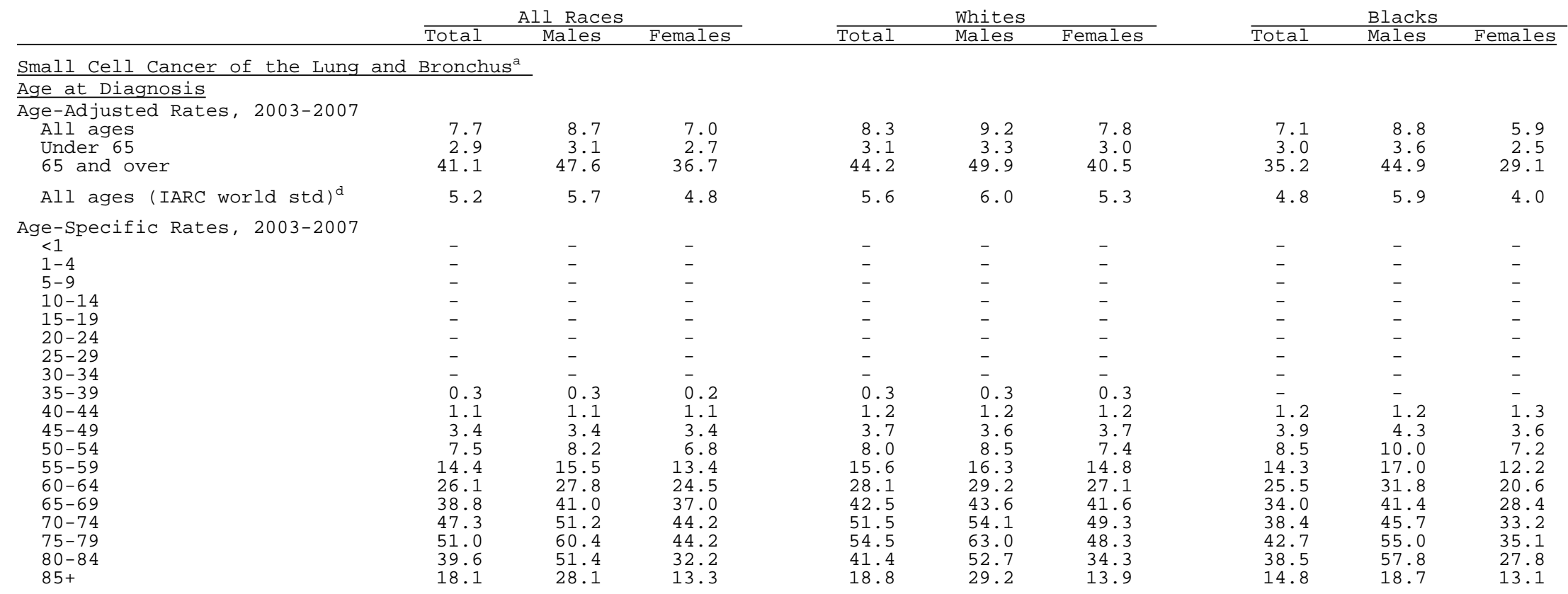

Non-Small Cell Cancer Lung and Bronchus ${ }^{b}$

\section{Age at Diagnosis}

Age-Adjusted Rates, 2003-2007

\section{All ages}

65 and ove

All ages (IARC world std) ${ }^{\text {b }}$

54.7
17.0
315.5

67.5
19.1
402.0

45.4
15.0

15.0
255.2

55.5
16.6

16.6
324.1

67.1

18.2
405.6

47.0

15.1
267.1

66.5

26.5

342.9

92.4

34.3

40.4

29.6

43.7

59.5

20.2
247.5

Age-Specific Rates, 2003-2007

$$
\begin{aligned}
& <1 \\
& 1-4 \\
& 5-9 \\
& 10-14 \\
& 15-19 \\
& 20-24 \\
& 25-29 \\
& 30-34 \\
& 35-39 \\
& 40-44 \\
& 45-49 \\
& 50-54 \\
& 55-59 \\
& 60-64 \\
& 65-69 \\
& 70-74 \\
& 75-79 \\
& 80-84 \\
& 85+
\end{aligned}
$$

\begin{tabular}{r}
\multicolumn{1}{r}{} \\
- \\
- \\
$\overline{0}$ \\
0.1 \\
0.3 \\
0.6 \\
1.2 \\
2.9 \\
8.8 \\
21.9 \\
42.4 \\
79.0 \\
146.4 \\
236.7 \\
324.1 \\
385.0 \\
376.0 \\
281.5
\end{tabular}
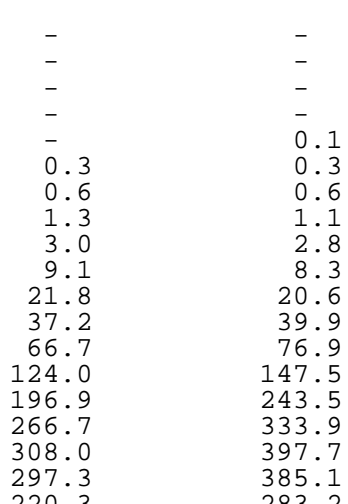

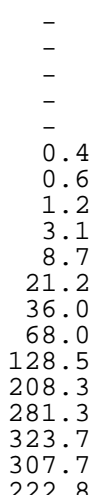

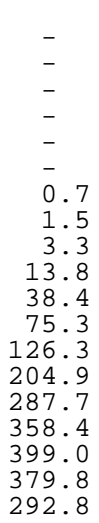

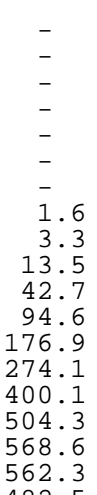

32.2

Small Cell Cancer of the Lung and Bronchus includes histologies 8041-8045.

Non-Small Cell Cancer of the Lung and Bronchus includes histologies 8000-8040, 8046-9049, 9056-9139, 9141-9589.

SEER 17 areas. Rates are per 100,000 and are age-adjusted to the 2000 US Std Population (19 age groups - Census P25-1130), unless noted.

Rates are per 100,000 and are age-adjusted to the IARC world standard population.

Statistic not shown. Rate based on less than 16 cases for the time interval. 


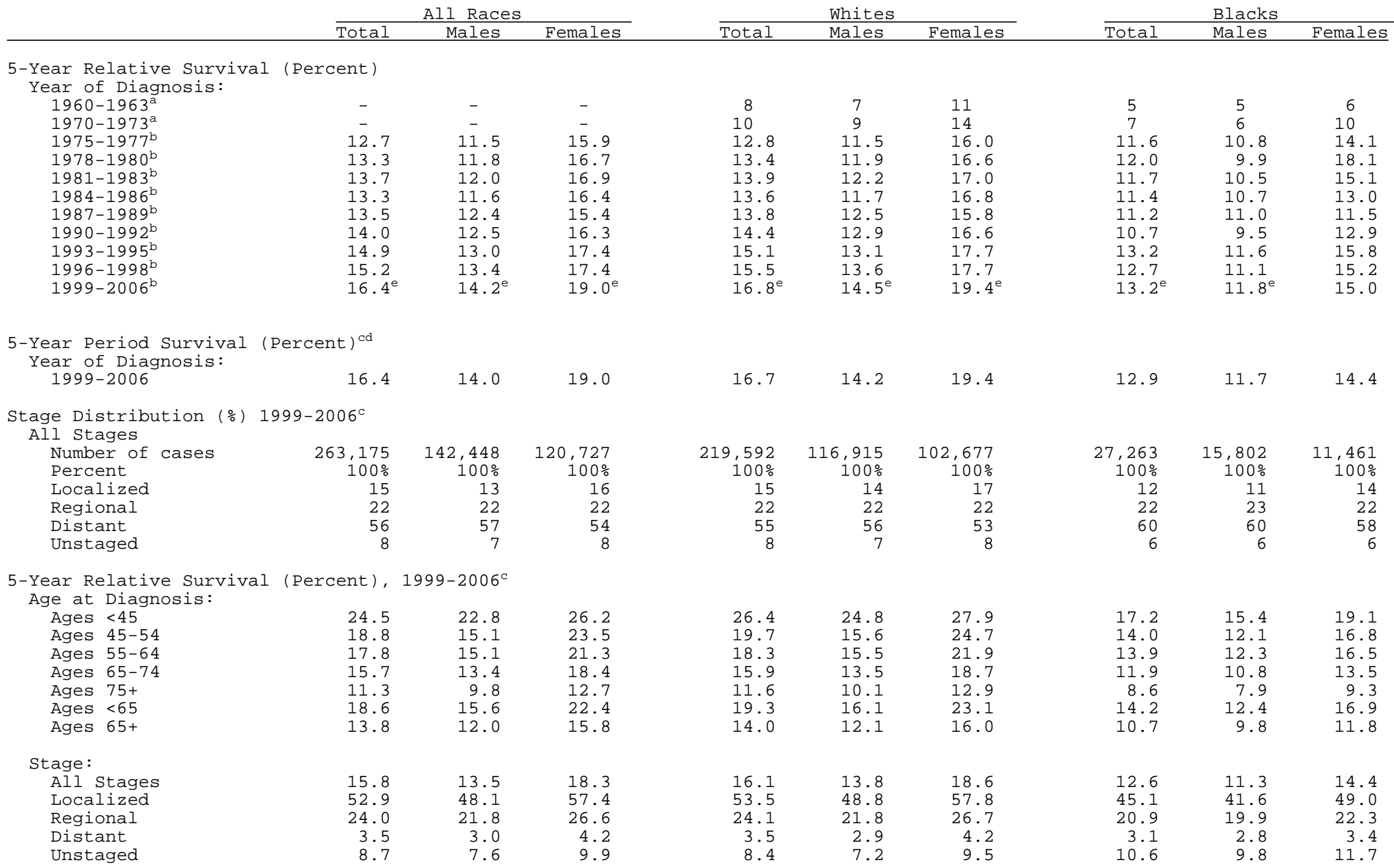

Based on End Results data from a series of hospital registries and one population-based registry.

Based on the SEER 9 areas (San Francisco, Connecticut, Detroit, Hawaii, Iowa, New Mexico, Seattle, Utah, Atlanta).

Based on follow-up of patients into 2007 . Connecticut, Detroit, Hawaii, Iowa, New Mexico, Seattle, Utah, Atlanta,

San Jose-Monterey, Los Angeles, Alaska Native Registry, Rural Georgia, California excluding SF/SJM/LA, Kentucky, Louisiana and New Jersey). California excluding SF/SJM/LA, Kentucky, Louisiana, and New Jersey contribute cases for diagnosis years $2000-2006$. The remaining 13 sen Areas contribute cases for the entire period 1999-2006. Based on follow-up of patients into 2007. Period survival provides a more up-to-date estimate of survival by piecing together the most recent conditional survival estimates

(2003-2005: 1-2 year survival), (2002-2004: 2-3 year survival), (2001-2003: 3-4 year survival), (2000-2002: 4-5 years survival). The difference between $1975-1977$ and $1999-2006$ is statistically significant (p<.05).

The standard error is between 5 and 10 percentage points.

Statistic could not be calculated due to fewer than 25 cases during the time period. 


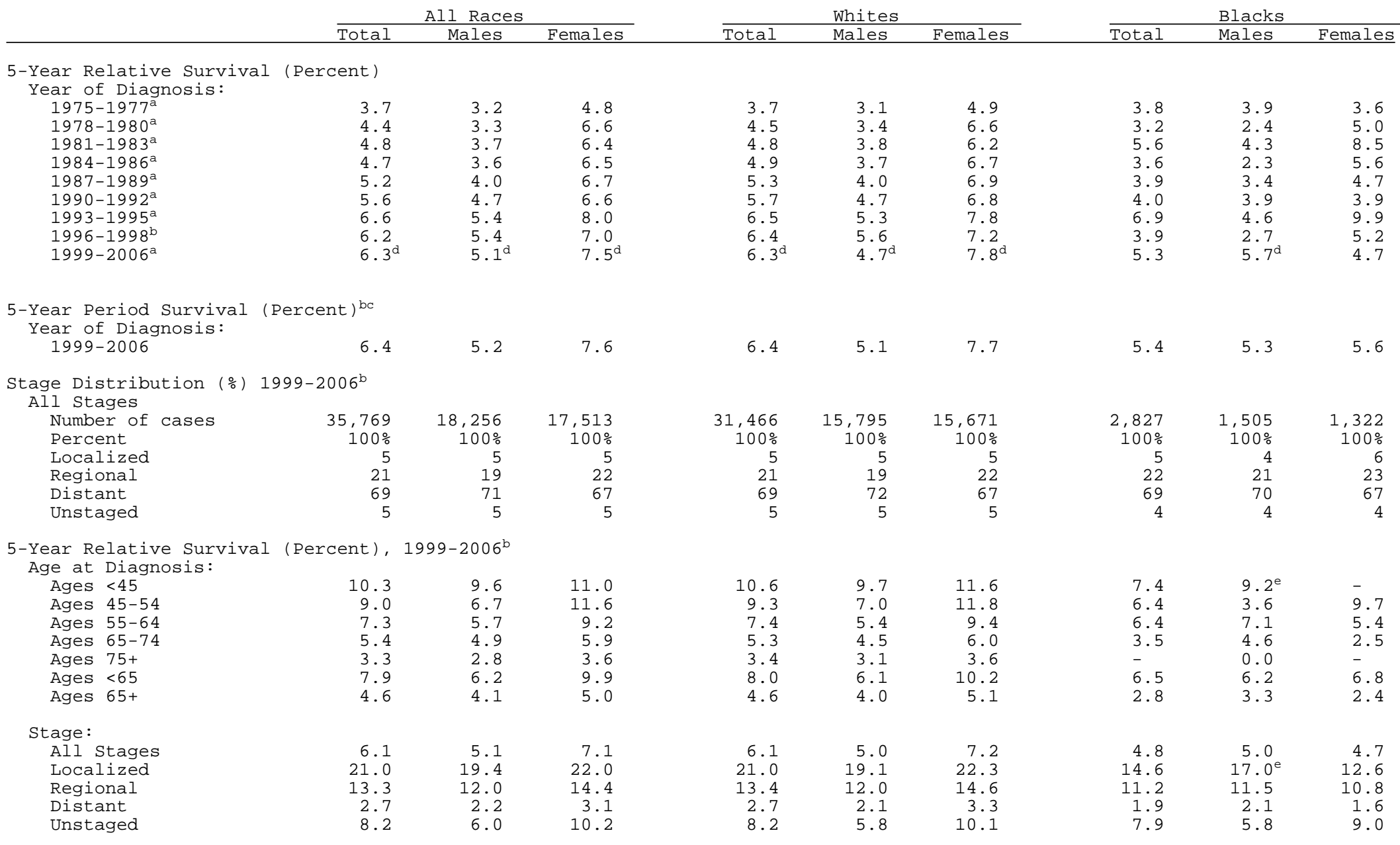

Based on the SEER 9 areas (San Francisco, Connecticut, Detroit, Hawaii, Iowa, New Mexico, Seattle, Utah, Atlanta). Based on follow-up of patients into 2007.

Based on the SEER 17 areas (San Francisco, Connecticut, Detroit, Hawaii, Iowa, New Mexico, Seattle, Utah, Atlanta,

San Jose-Monterey, Los Angeles, Alaska Native Registry, Rural Georgia, California excluding SF/SJM/LA, Kentucky, Louisiana and New Jersey). California excluding SF/SJM/LA, Kentucky, Louisiana, and New Jersey contribute cases for diagnosis years $2000-2006$. Period survival provides a more up-to-date estimate of survival by piecing together the most recent conditional survival estimates (2003-2005: 1-2 year. survival) (2002-2004: 2-3 year survival), (2001-2003: 3-

(2003-2005: 1-2 year survival), (2002-2004: 2-3 year survival), (2001-2003: 3-4 year survival), (2000-2002: 4-5 years survival). The difference between 1975-1977 and 1999-2006 is statistically significant (p<.05).

The standard error is between 5 and 10 percentage points.

Statistic could not be calculated due to fewer than 25 cases during the time period. 


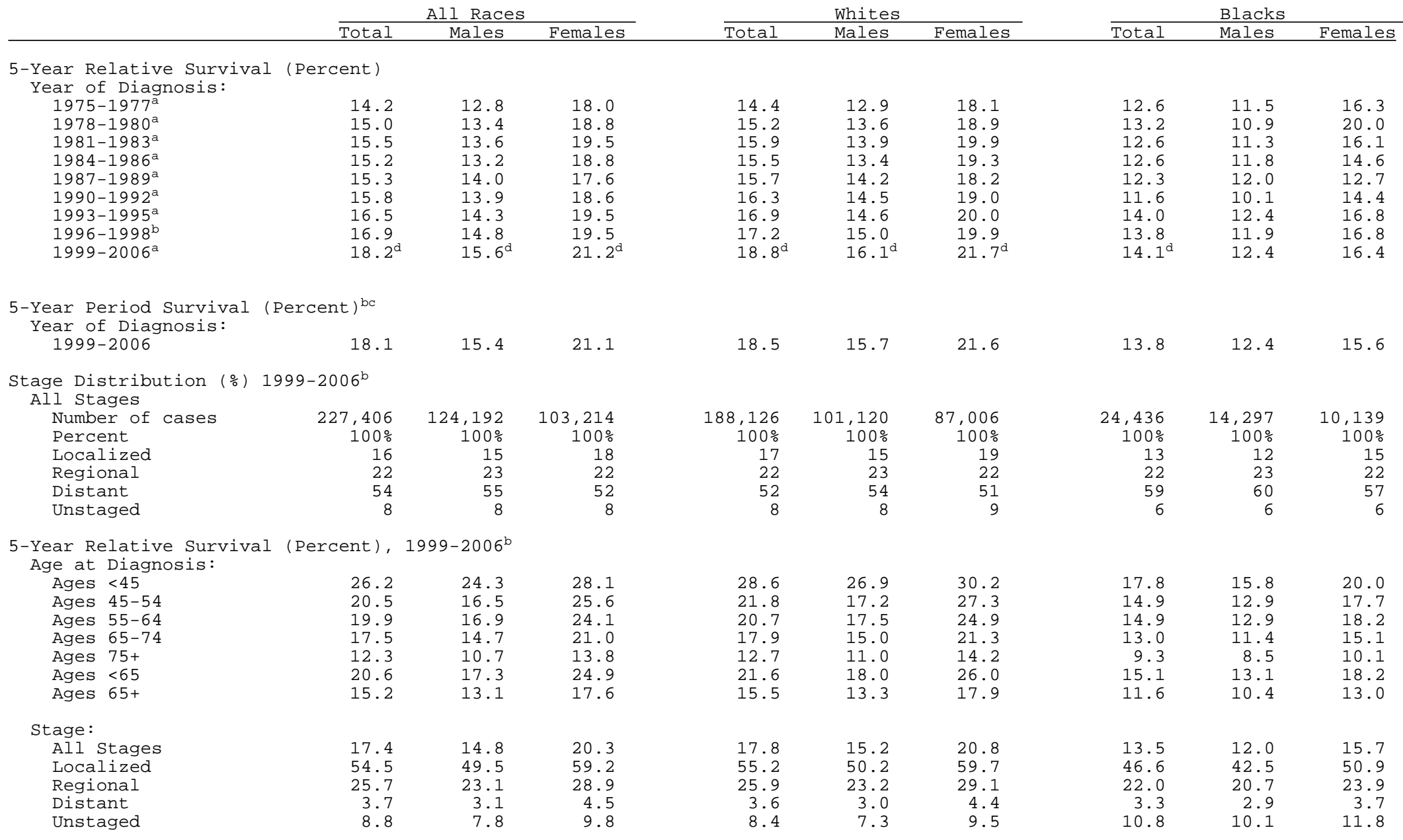

Based on the SEER 9 areas (San Francisco, Connecticut, Detroit, Hawaii, Iowa, New Mexico, Seattle, Utah, Atlanta). Based on follow-up of patients into 2007

Based on the SEER 17 areas (San Francisco, Connecticut, Detroit, Hawaii, Iowa, New Mexico, Seattle, Utah, Atlanta,

San Jose-Monterey, Los Angeles, Alaska Native Registry, Rural Georgia, California excluding SF/SJM/LA, Kentucky, Louisiana and New Jersey). California excluding SF/SJM/LA, Kentucky, Louisiana, and New Jersey contribute cases for diagnosis years $2000-2006$. The remaining 13 segR Areas contribute cases for the entire period 1999-2006. Based on follow-up of pati Period survival provides a more up-to-date estimate of survival by piecing together the most recent conditional survival estimates (2003-2005: 1-2 year survival), (2002-2004: 2-3 year survival), (2001-2003: 3-4 year survival), (2000-2002: 4-5 years survival). The difference between 1975-1977 and 1999-2006 is statistically significant (p<.05). The standard error is between 5 and 10 percentage points.

Statistic could not be calculated due to fewer than 25 cases during the time period. 
Table 15.15

Cancer of the Lung and Bronchus (Invasive)

SEER Relative Survival (Percent)

By Year of Diagnosis

All Races, Males and Females

Year of Diagnosis

$1975-1980-1985-$

\begin{tabular}{|c|c|c|c|c|c|c|c|c|c|c|c|c|c|c|c|c|c|c|c|c|}
\hline & 1979 & 1984 & 1989 & 1990 & 1991 & 1992 & 1993 & 1994 & 1995 & 1996 & 1997 & 1998 & 1999 & 2000 & 2001 & 2002 & 2003 & 2004 & 2005 & 2006 \\
\hline Survival & Time & & & & & & & & & & & & & & & & & & & \\
\hline 1-year & 36.7 & 38.7 & 40.0 & 40.3 & 41.2 & 41.0 & 40.8 & 40.9 & 41.5 & 41.1 & 41.7 & 41.3 & 42.7 & 42.4 & 41.6 & 42.0 & 43.1 & 43.5 & 44.5 & 44.9 \\
\hline 2-year & 21.7 & 22.5 & 23.1 & 23.6 & 23.9 & 24.7 & 24.5 & 24.5 & 25.4 & 25.3 & 25.4 & 25.9 & 26.4 & 26.6 & 26.3 & 27.1 & 27.1 & 28.1 & 28.6 & \\
\hline 3-year & 16.7 & 17.4 & 17.5 & 18.0 & 18.7 & 18.9 & 18.9 & 19.1 & 19.6 & 19.8 & 19.8 & 20.3 & 20.3 & 20.9 & 20.4 & 21.4 & 21.2 & 22.7 & & \\
\hline 4-year & 14.4 & 14.9 & 15.0 & 15.3 & 15.9 & 16.2 & 16.2 & 16.4 & 17.0 & 16.8 & 17.0 & 17.5 & 17.4 & 18.1 & 17.4 & 18.1 & 18.3 & & & \\
\hline 5-year & 13.0 & 13.4 & 13.5 & 13.7 & 14.1 & 14.3 & 14.7 & 14.8 & 15.1 & 15.2 & 15.3 & 15.2 & 15.7 & 16.3 & 15.6 & 16.2 & & & & \\
\hline 6-year & 12.0 & 12.3 & 12.4 & 12.7 & 13.1 & 13.2 & 13.6 & 13.6 & 13.8 & 13.9 & 13.9 & 13.9 & 14.4 & 14.8 & 14.1 & & & & & \\
\hline 7-year & 11.4 & 11.5 & 11.5 & 11.7 & 12.0 & 12.2 & 12.6 & 12.7 & 13.1 & 12.9 & 12.9 & 12.9 & 13.3 & 13.6 & & & & & & \\
\hline 8 -year & 10.8 & 10.7 & 10.7 & 11.0 & 11.2 & 11.3 & 11.8 & 12.0 & 12.3 & 12.0 & 12.0 & 11.9 & 12.6 & & & & & & & \\
\hline 9-year & 10.2 & 10.0 & 10.1 & 10.1 & 10.6 & 10.6 & 11.1 & 11.3 & 11.6 & 11.3 & 11.1 & 11.3 & & & & & & & & \\
\hline 10-year & 9.6 & 9.4 & 9.6 & 9.4 & 10.1 & 10.0 & 10.4 & 10.7 & 11.0 & 10.4 & 10.6 & & & & & & & & & \\
\hline 11-year & 9.2 & 9.1 & 9.1 & 8.9 & 9.5 & 9.4 & 9.8 & 10.1 & 10.5 & 9.9 & & & & & & & & & & \\
\hline 12-year & 8.9 & 8.6 & 8.6 & 8.3 & 9.0 & 9.1 & 9.2 & 9.4 & 10.0 & & & & & & & & & & & \\
\hline 13-year & 8.5 & 8.2 & 8.2 & 8.1 & 8.4 & 8.6 & 8.8 & 9.0 & & & & & & & & & & & & \\
\hline 14-year & 8.2 & 7.9 & 7.8 & 7.9 & 7.8 & 8.4 & 8.5 & & & & & & & & & & & & & \\
\hline 15-year & 7.8 & 7.5 & 7.5 & 7.6 & 7.4 & 7.9 & & & & & & & & & & & & & & \\
\hline 16-year & 7.6 & 7.2 & 7.2 & 7.2 & 6.9 & & & & & & & & & & & & & & & \\
\hline 17-year & 7.3 & 6.9 & 6.9 & 6.9 & & & & & & & & & & & & & & & & \\
\hline 18-year & 7.0 & 6.6 & 6.6 & & & & & & & & & & & & & & & & & \\
\hline 19-year & 6.8 & 6.2 & & & & & & & & & & & & & & & & & & \\
\hline 20-year & 6.6 & 5.9 & & & & & & & & & & & & & & & & & & \\
\hline
\end{tabular}


Table 15.16

Cancer of the Lung and Bronchus (Invasive)

SEER ${ }^{a}$ Relative Survival (Percent)

By Year of Diagnosis

All Races, Males

Year of Diagnosis

$1975-1980-\quad 1985-$ $1984 \quad 1989$

$\begin{array}{lllllllll}1990 & 1991 & 1992 & 1993 & 1994 & 1995 & 1996 & 1997 & 1998\end{array}$

19992000

2001

2002

$2003 \quad 2004 \quad 2005$

Survival Time

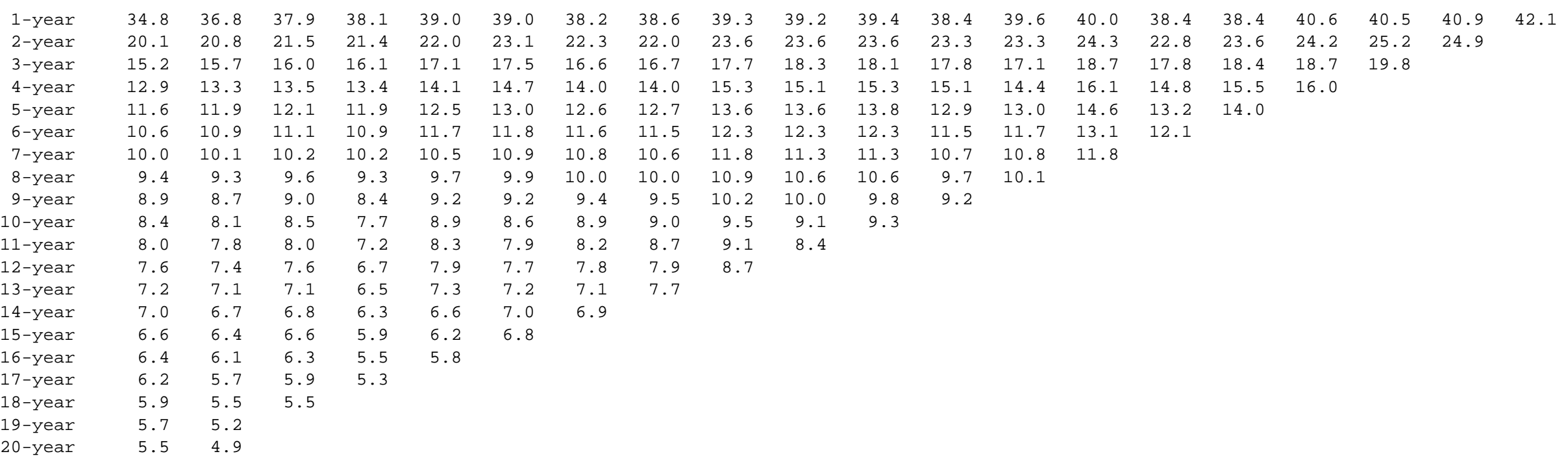


Table 15.17

Cancer of the Lung and Bronchus (Invasive)

SEER Relative Survival (Percent)

By Year of Diagnosis

All Races, Females

Year of Diagnosis

$1975-1980-1985-$

\begin{tabular}{|c|c|c|c|c|c|c|c|c|c|c|c|c|c|c|c|c|c|c|c|c|}
\hline & 1979 & 1984 & 1989 & 1990 & 1991 & 1992 & 1993 & 1994 & 1995 & 1996 & 1997 & 1998 & 1999 & 2000 & 2001 & 2002 & 2003 & 2004 & 2005 & 2006 \\
\hline Survival & Time & & & & & & & & & & & & & & & & & & & \\
\hline 1-year & 41.8 & 42.7 & 43.7 & 43.7 & 44.5 & 44.1 & 44.5 & 44.1 & 44.6 & 43.5 & 44.6 & 44.9 & 46.5 & 45.3 & 45.7 & 46.3 & 46.0 & 46.9 & 48.5 & 48.0 \\
\hline 2-year & 25.8 & 26.2 & 26.0 & 27.0 & 26.8 & 27.0 & 27.8 & 27.9 & 27.9 & 27.6 & 27.6 & 29.1 & 30.2 & 29.4 & 30.5 & 31.1 & 30.4 & 31.5 & 32.6 & \\
\hline 3-year & 20.6 & 20.9 & 20.1 & 20.9 & 21.1 & 21.1 & 22.2 & 22.2 & 22.1 & 21.8 & 21.8 & 23.3 & 24.1 & 23.6 & 23.6 & 24.8 & 24.1 & 25.9 & & \\
\hline 4-year & 18.2 & 18.1 & 17.6 & 18.0 & 18.6 & 18.3 & 19.2 & 19.4 & 19.4 & 18.9 & 19.1 & 20.4 & 21.0 & 20.5 & 20.5 & 21.1 & 21.1 & & & \\
\hline 5-year & 16.6 & 16.4 & 15.8 & 16.4 & 16.4 & 16.1 & 17.6 & 17.5 & 17.1 & 17.2 & 17.1 & 18.0 & 18.9 & 18.3 & 18.5 & 18.8 & & & & \\
\hline 6-year & 15.4 & 15.1 & 14.6 & 15.2 & 15.2 & 15.1 & 16.3 & 16.4 & 15.7 & 15.8 & 15.7 & 16.7 & 17.4 & 16.8 & 16.6 & & & & & \\
\hline 7-year & 14.5 & 14.2 & 13.5 & 14.0 & 14.1 & 14.0 & 15.1 & 15.2 & 14.8 & 14.9 & 14.7 & 15.6 & 16.2 & 15.7 & & & & & & \\
\hline 8 -year & 13.8 & 13.4 & 12.6 & 13.3 & 13.2 & 13.3 & 14.2 & 14.5 & 14.1 & 13.8 & 13.6 & 14.7 & 15.4 & & & & & & & \\
\hline 9 -year & 13.1 & 12.5 & 11.8 & 12.5 & 12.6 & 12.5 & 13.4 & 13.4 & 13.4 & 12.8 & 12.7 & 13.9 & & & & & & & & \\
\hline 10-year & 12.3 & 11.8 & 11.2 & 11.7 & 11.8 & 11.9 & 12.3 & 12.7 & 12.8 & 12.0 & 12.1 & & & & & & & & & \\
\hline 11-year & 11.9 & 11.3 & 10.6 & 11.2 & 11.2 & 11.3 & 11.8 & 11.9 & 12.3 & 11.8 & & & & & & & & & & \\
\hline 12-year & 11.5 & 10.7 & 10.1 & 10.5 & 10.5 & 10.9 & 11.1 & 11.1 & 11.6 & & & & & & & & & & & \\
\hline 13-year & 11.1 & 10.2 & 9.7 & 10.3 & 9.8 & 10.4 & 10.9 & 10.6 & & & & & & & & & & & & \\
\hline 14-year & 10.6 & 9.8 & 9.3 & 9.9 & 9.2 & 10.0 & 10.5 & & & & & & & & & & & & & \\
\hline 15-year & 10.1 & 9.4 & 8.9 & 9.8 & 8.8 & 9.4 & & & & & & & & & & & & & & \\
\hline 16-year & 9.9 & 9.0 & 8.5 & 9.4 & 8.2 & & & & & & & & & & & & & & & \\
\hline 17-year & 9.4 & 8.7 & 8.3 & 9.0 & & & & & & & & & & & & & & & & \\
\hline 18-year & 9.1 & 8.3 & 8.0 & & & & & & & & & & & & & & & & & \\
\hline 19-year & 8.7 & 7.7 & & & & & & & & & & & & & & & & & & \\
\hline 20-year & 8.5 & 7.4 & & & & & & & & & & & & & & & & & & \\
\hline
\end{tabular}


Risk of Being Diagnosed With Cancer in 10, 20 and 30 Years,

Lifetime Risk of Being Diagnosed with Cancer Given Alive and Cancer-Free at Current Age, and

Lifetime Risk of Dying from Cancer Given Alive at Current Age Both Sexes, 2005-2007 By Race/Ethnicity

Risk of Being

Race/ Current

Diagnosed with Cancer
Risk of Dying Ethnicity $+10 \mathrm{v}$

All Races

0
10
20
30
40
50
60
70
80

White

0
10
20
30
40
50
60
70
80

0.00
0.00
0.00
0.02
0.18
0.69
2.00
3.33
2.75

20 yrs

$\begin{array}{ll}0.00 & 0.00 \\ 0.00 & 0.01 \\ 0.00 & 0.03 \\ 0.02 & 0.19 \\ 0.17 & 0.83 \\ 0.68 & 2.62 \\ 2.06 & 5.07 \\ 3.45 & 5.46 \\ 2.80 & -\end{array}$

Black

$\begin{array}{rll}0 & 0.00 & 0.00 \\ 10 & 0.00 & 0.00 \\ 20 & 0.00 & 0.03 \\ 30 & 0.03 & 0.30 \\ 40 & 0.28 & 1.26 \\ 50 & 1.03 & 3.16 \\ 60 & 2.39 & 5.02 \\ 70 & 3.28 & 4.98 \\ 80 & 2.63 & -\end{array}$

$0.00 \quad 0.01$

Ever
from Cancer

0.01

0.01

6.95

5.93

0.01
0.03

0.20

0.20

0.86

6.95
7.03

$$
6.01
$$

6.01
6.04

6.10

6.16

0.85
2.57

2.67

7.12

$5.30-7.19$

6.20

4.90

6.61

6.94

5.68

6.01

\begin{tabular}{l}
$-\quad 3.27$ \\
\hline
\end{tabular}

3.08

$0.01 \quad 7.15$

$0.03 \quad 7.23$

$0.19 \quad 7.25$

$0.85 \quad 7.31$

\subsection{8}

6.15

6.18

6.23

$2.72 \quad 7.38$

$5.46 \quad 7.40$

$\begin{array}{ll}6.82 & 7.13\end{array}$

6.29

6.33

6.13

5.11

5.81
$-\quad 3.30$

3.11

$0.00 \quad 6.53$

$0.03 \quad 6.68$

0.30

6.72

5.41

1.26

3.29

5.52

6.83

7.01

5.55
5.58

5.58

5.77

5.84

5.64

$5.35 \quad 4.64$

Devcan Version 6.5.0, June 2010, National Cancer Institute (http://srab.cancer.gov/devcan/).

Source: Incidence data are from the SEER 17 areas (San Francisco, Connecticut, Detroit, Hawaii, Iowa, New Mexico, Seattle, Utah, Atlanta, San Jose-Monterey, Los Angeles, Alaska Native Registry, Rural Georgia, California excluding SF/SJM/LA, Kentucky, Louisiana and New Jersey). Mortality data are from the NCHS public use data file for the total US.

Underlying incidence and mortality data for American Indian/Alaska Native are based on the CHSDA(Contract Health Service Delivery Area) counties.

Hispanic is not mutually exclusive from whites, blacks, Asian/Pacific Islanders, and American Indians/Alaska Natives.

Underlying incidence data for Hispanics are based on NHIA and exclude cases from the Alaska Native Registry.

Underlying mortality data for Hispanics exclude deaths from the District of Columbia, Minnesota, New Hampsire and North Dakota.

A percent of 0.00 represents a value that is below 0.005 . 
Risk of Being Diagnosed With Cancer in 10, 20 and 30 Years,

Lifetime Risk of Being Diagnosed with Cancer Given Alive and Cancer-Free at Current Age, and

Lifetime Risk of Dying from Cancer Given Alive at Current Age Males, 2005-2007 By Race/Ethnicity

Risk of Being

Race/ Current Diagnosed with Cancer Risk of Dying $+10 \mathrm{yr}$

All Races

0
10
20
30
40
50
60
70
80

White

0
10
20
30
40
50
60
70
80

0.00
0.00
0.00
0.02
0.17
0.78
2.29
3.96
3.48

20 yrs

0.00
0.00
0.00
0.02
0.1
0.7
2.30
4.02
3.5

Black

$\begin{array}{rlllll}0 & 0.00 & 0.00 & 0.01 & 7.65 & 6.83 \\ 10 & 0.00 & 0.01 & 0.04 & 7.85 & 7.02 \\ 20 & 0.00 & 0.04 & 0.32 & 7.92 & 7.07 \\ 30 & 0.03 & 0.32 & 1.52 & 8.11 & 7.23 \\ 40 & 0.30 & 1.54 & 4.10 & 8.33 & 7.43 \\ 50 & 1.32 & 4.04 & 6.87 & 8.53 & 7.63 \\ 60 & 3.14 & 6.41 & 7.98 & 8.32 & 7.55 \\ 70 & 4.33 & 6.40 & - & 6.86 & 6.44 \\ 80 & 3.59 & - & - & 4.39 & 4.43\end{array}$

Risk of Being

\begin{tabular}{|c|c|c|c|c|c|c|}
\hline \multirow{2}{*}{$\begin{array}{l}\text { Race/ } \\
\text { Ethnicity }\end{array}$} & \multirow{2}{*}{$\begin{array}{l}\text { Current } \\
\text { Age }\end{array}$} & \multicolumn{4}{|c|}{ Diagnosed with Cancer } & \multirow{2}{*}{$\begin{array}{l}\text { Risk of Dying } \\
\text { from Cancer }\end{array}$} \\
\hline & & $+10 \mathrm{yrs}$ & $+20 \mathrm{yrs}$ & $+30 \mathrm{yrs}$ & Ever & \\
\hline Asian/ & 0 & 0.00 & 0.00 & 0.01 & 6.92 & 5.50 \\
\hline Pacific & 10 & 0.00 & 0.01 & 0.03 & 6.99 & 5.55 \\
\hline \multirow{7}{*}{ Islander } & 20 & 0.01 & 0.03 & 0.16 & 7.01 & 5.56 \\
\hline & 30 & 0.03 & 0.15 & 0.67 & 7.05 & 5.60 \\
\hline & 40 & 0.13 & 0.65 & 1.99 & 7.08 & 5.62 \\
\hline & 50 & 0.53 & 1.89 & 4.48 & 7.07 & 5.62 \\
\hline & 60 & 1.42 & 4.12 & 6.20 & 6.84 & 5.51 \\
\hline & 70 & 2.99 & 5.29 & - & 6.01 & 5.06 \\
\hline & 80 & 2.99 & - & - & 3.91 & 3.86 \\
\hline American & 0 & 0.00 & 0.01 & 0.01 & 5.01 & 4.32 \\
\hline Indian/ & 10 & 0.01 & 0.01 & 0.03 & 5.10 & 4.40 \\
\hline Alaska & 20 & 0.01 & 0.03 & 0.09 & 5.14 & 4.45 \\
\hline \multirow[t]{6}{*}{ Native ${ }^{a}$} & 30 & 0.02 & 0.09 & 0.55 & 5.27 & 4.57 \\
\hline & 40 & 0.07 & 0.55 & 1.66 & 5.44 & 4.75 \\
\hline & 50 & 0.50 & 1.67 & 4.14 & 5.67 & 4.99 \\
\hline & 60 & 1.29 & 4.00 & 5.25 & 5.69 & 5.09 \\
\hline & 70 & 3.25 & 4.76 & - & 5.28 & 4.45 \\
\hline & 80 & 2.21 & - & - & 2.98 & 2.72 \\
\hline \multirow[t]{9}{*}{ Hispanic ${ }^{b}$} & 0 & 0.00 & 0.00 & 0.00 & 4.95 & 4.07 \\
\hline & 10 & 0.00 & 0.00 & 0.01 & 5.01 & 4.12 \\
\hline & 20 & 0.00 & 0.01 & 0.08 & 5.04 & 4.14 \\
\hline & 30 & 0.01 & 0.08 & 0.38 & 5.10 & 4.19 \\
\hline & 40 & 0.07 & 0.37 & 1.41 & 5.16 & 4.24 \\
\hline & 50 & 0.32 & 1.39 & 3.29 & 5.24 & 4.31 \\
\hline & 60 & 1.14 & 3.17 & 4.69 & 5.25 & 4.32 \\
\hline & 70 & 2.33 & 4.07 & - & 4.71 & 3.93 \\
\hline & 80 & 2.39 & - & - & 3.28 & 2.93 \\
\hline
\end{tabular}

Devcan Version 6.5.0, June 2010, National Cancer Institute (http://srab.cancer.gov/devcan/).

Source: Incidence data are from the SEER 17 areas (San Francisco, Connecticut, Detroit, Hawaii, Iowa, New Mexico, Seattle, Utah, Atlanta, San Jose-Monterey, Los Angeles, Alaska Native Registry, Rural Georgia, California excluding SF/SJM/LA, Kentucky, Louisiana and New Jersey). Mortality data are from the NCHS public use data file for the total US.

Underlying incidence and mortality data for American Indian/Alaska Native are based on the CHSDA(Contract Health Service Delivery Area) counties.

Hispanic is not mutually exclusive from whites, blacks, Asian/Pacific Islanders, and American Indians/Alaska Natives.

Underlying incidence data for Hispanics are based on NHIA and exclude cases from the Alaska Native Registry.

Underlying mortality data for Hispanics exclude deaths from the District of Columbia, Minnesota, New Hampshire and North Dakota.

Statistic could not be calculated.

A percent of 0.00 represents a value that is below 0.005 . 
Table 15.20

Cancer of the Lung and Bronchus (Invasive)

Risk of Being Diagnosed With Cancer in 10, 20 and 30 Years

Lifetime Risk of Being Diagnosed with Cancer Given Alive and Cancer-Free at Current Age, and

Lifetime Risk of Dying from Cancer Given Alive at Current Age

Females, 2005-2007 By Race/Ethnicity

Risk of Being

Race/ Current Diagnosed with Cancer Risk of Dying $+10 \mathrm{yr}$

All Races

0
10
20
30
40
50
60
70
80

White

0
10
20
30
40
50
60
70
80

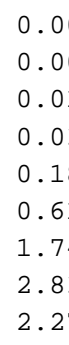

0.00

yrs

0.00

0.01
Ever from Cancer

0.01

0.03

6.35

5.05

0.03

0.20

0.21

0.79

2.40

6.42

6.43
6.46

6.46
6.49

2.27

4.68

5.80

6.44

6.12

4.90

2.74

$\begin{array}{ll}0.00 & 0.00 \\ 0.00 & 0.01 \\ 0.01 & 0.03 \\ 0.03 & 0.20 \\ 0.18 & 0.78 \\ 0.62 & 2.38 \\ 1.84 & 4.52 \\ 2.99 & 4.76 \\ 2.34 & -\end{array}$

\subsection{1}

0.03

0.20

0.80

2.51

4.94

6.10$$
-
$$

0.00

$0.00 \quad 0.00$

$0.00 \quad 0.03$

$0.03 \quad 0.29$

$0.26 \quad 1.01$

$0.78 \quad 2.41$

$\begin{array}{ll}1.78 & 3.91\end{array}$

$2.52 \quad 3.97$

2.08

80

Devcan Version 6.5.0, June 2010, National Cancer Institute (http://srab.cancer.gov/devcan/).

Source: Incidence data are from the SEER 17 areas (San Francisco, Connecticut, Detroit, Hawaii, Iowa, New Mexico, Seattle, Utah, Atlanta, San Jose-Monterey, Los Angeles, Alaska Native Registry, Rural Georgia, California excluding SF/SJM/LA, Kentucky, Louisiana and New Jersey). Mortality data are from the NCHS public use data file for the total US.

Underlying incidence and mortality data for American Indian/Alaska Native are based on the CHSDA(Contract Health Service Delivery Area) counties.

Hispanic is not mutually exclusive from whites, blacks, Asian/Pacific Islanders, and American Indians/Alaska Natives.

Underlying incidence data for Hispanics are based on NHIA and exclude cases from the Alaska Native Registry.

Underlying mortality data for Hispanics exclude deaths from the District of Columbia, Minnesota, New Hampsire and North Dakota.

A percent of 0.00 represents a value that is below 0.005 .

Risk of Being

\begin{tabular}{|c|c|c|c|c|c|c|}
\hline \multirow{2}{*}{$\begin{array}{l}\text { Race/ } \\
\text { Ethnicity }\end{array}$} & \multirow{2}{*}{$\begin{array}{l}\text { Current } \\
\text { Age }\end{array}$} & \multicolumn{4}{|c|}{ Diagnosed with Cancer } & \multirow{2}{*}{$\begin{array}{l}\text { Risk of Dying } \\
\text { from Cancer }\end{array}$} \\
\hline & & +10 yrs & +20 yrs & +30 yrs & Ever & \\
\hline Asian/ & 0 & 0.00 & 0.00 & 0.00 & 4.40 & 3.25 \\
\hline Pacific & 10 & 0.00 & 0.00 & 0.03 & 4.43 & 3.28 \\
\hline \multirow[t]{7}{*}{ Islander } & 20 & 0.00 & 0.03 & 0.13 & 4.44 & 3.28 \\
\hline & 30 & 0.02 & 0.13 & 0.46 & 4.45 & 3.29 \\
\hline & 40 & 0.10 & 0.43 & 1.23 & 4.44 & 3.29 \\
\hline & 50 & 0.33 & 1.14 & 2.55 & 4.39 & 3.26 \\
\hline & 60 & 0.83 & 2.27 & 3.49 & 4.16 & 3.14 \\
\hline & 70 & 1.54 & 2.84 & - & 3.55 & 2.82 \\
\hline & 80 & 1.53 & - & - & 2.38 & 2.10 \\
\hline American & 0 & 0.00 & 0.00 & 0.01 & 4.54 & 3.78 \\
\hline Indian/ & 10 & 0.00 & 0.01 & 0.02 & 4.59 & 3.83 \\
\hline Alaska & 20 & 0.00 & 0.02 & 0.11 & 4.61 & 3.85 \\
\hline \multirow[t]{6}{*}{ Native ${ }^{a}$} & 30 & 0.02 & 0.11 & 0.48 & 4.65 & 3.89 \\
\hline & 40 & 0.09 & 0.47 & 1.54 & 4.72 & 3.96 \\
\hline & 50 & 0.39 & 1.49 & 3.42 & 4.77 & 4.01 \\
\hline & 60 & 1.17 & 3.21 & 4.19 & 4.63 & 3.94 \\
\hline & 70 & 2.33 & 3.44 & - & 3.95 & 3.18 \\
\hline & 80 & 1.49 & - & - & 2.17 & 1.76 \\
\hline \multirow[t]{9}{*}{ Hispanic ${ }^{b}$} & 0 & 0.00 & 0.00 & 0.00 & 3.47 & 2.21 \\
\hline & 10 & 0.00 & 0.00 & 0.02 & 3.50 & 2.23 \\
\hline & 20 & 0.00 & 0.02 & 0.09 & 3.51 & 2.24 \\
\hline & 30 & 0.01 & 0.09 & 0.33 & 3.52 & 2.24 \\
\hline & 40 & 0.07 & 0.32 & 1.05 & 3.53 & 2.25 \\
\hline & 50 & 0.25 & 1.00 & 2.24 & 3.51 & 2.25 \\
\hline & 60 & 0.77 & 2.06 & 3.02 & 3.38 & 2.19 \\
\hline & 70 & 1.40 & 2.45 & - & 2.84 & 1.94 \\
\hline & 80 & 1.30 & - & - & 1.79 & 1.38 \\
\hline
\end{tabular}


Table 15.21

Lung and Bronchus (Invasive)

SEER Incidence and U.S. Mortality

Age-Adjusted Rates and Trends ${ }^{a}$

By Race/Ethnicity and Sex

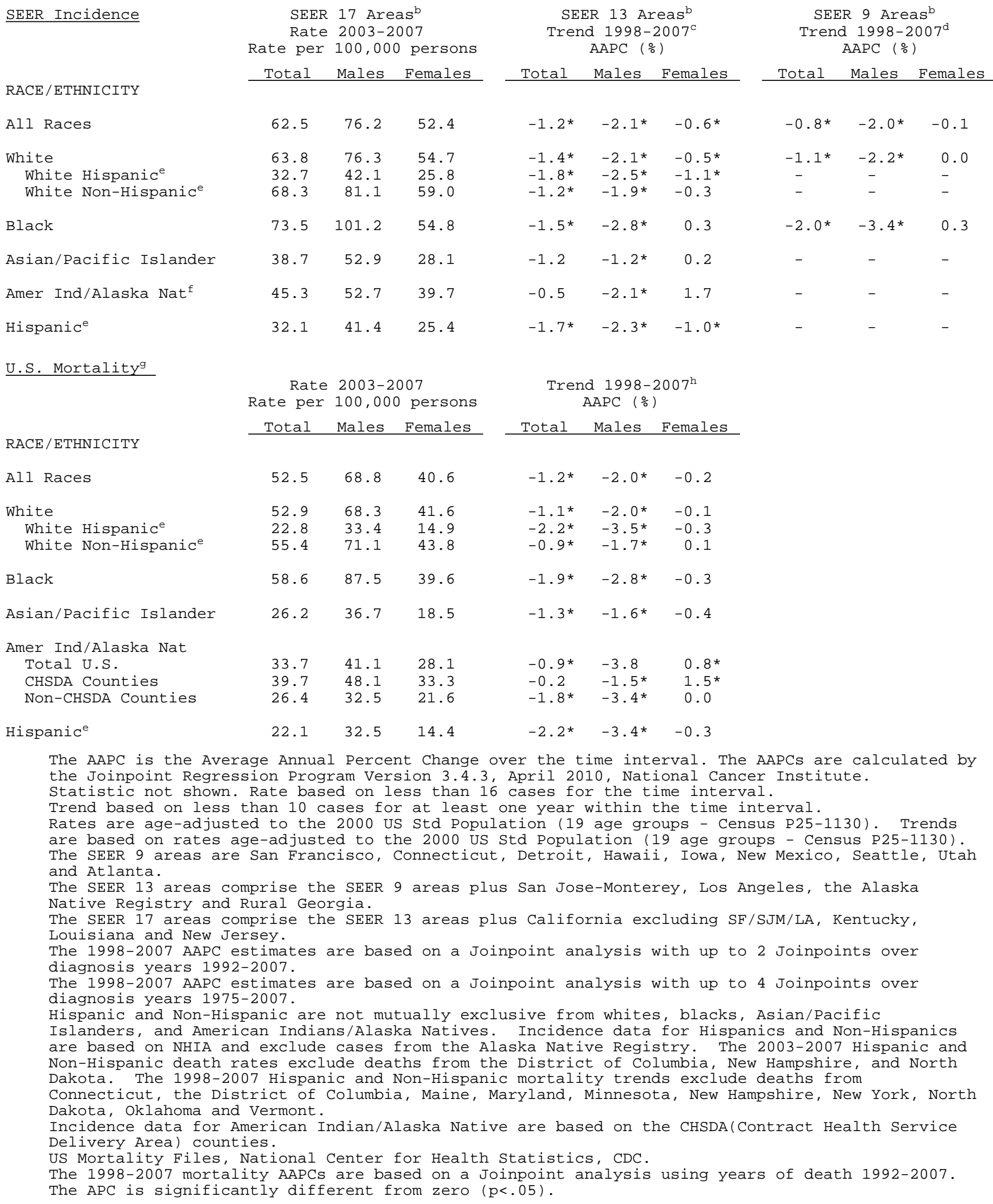


Table 15.22

Cancer of the Lung And Bronchus (Invasive)

Age-Adjusted SEER Incidence Rates ${ }^{a}$

By Registry, Race and Sex

\begin{tabular}{ccc}
\multicolumn{3}{c}{ All Races } \\
Total Males Females
\end{tabular}

Total

Whites

Females

Total

Blacks

Females

SEER Incidence Rates ${ }^{a}, 2003-2007$

\begin{tabular}{|c|c|c|c|c|c|c|c|c|c|}
\hline Atlanta \& Rural Georgia & 58.39 & 75.52 & 46.62 & 58.75 & 71.90 & 49.66 & 60.47 & 90.62 & 42.14 \\
\hline Atlanta & 57.68 & 73.83 & 46.68 & 57.55 & 69.56 & 49.42 & 61.08 & 91.08 & 42.93 \\
\hline Rural Georgia & 73.77 & 107.22 & 48.43 & 82.56 & 114.12 & 56.53 & 56.78 & 91.39 & 34.57 \\
\hline California & 53.74 & 63.92 & 46.28 & 55.21 & 64.12 & 48.74 & 69.09 & 88.38 & 55.50 \\
\hline Greater Bay Area & 50.71 & 59.04 & 44.75 & 51.84 & 57.32 & 48.19 & 72.87 & 89.46 & 61.31 \\
\hline San Francisco-Oakland & 52.33 & 60.81 & 46.30 & 52.69 & 57.63 & 49.47 & 75.19 & 91.73 & 63.68 \\
\hline San Jose-Monterey & 47.55 & 55.62 & 41.72 & 50.40 & 56.85 & 45.97 & 59.18 & 81.76 & 42.08 \\
\hline Los Angeles & 46.32 & 57.35 & 38.30 & 45.47 & 54.59 & 38.87 & 68.21 & 89.50 & 53.83 \\
\hline Greater California & 58.41 & 68.73 & 50.75 & 60.17 & 69.93 & 52.99 & 67.87 & 86.67 & 53.79 \\
\hline Connecticut & 68.42 & 80.50 & 60.25 & 69.34 & 80.53 & 61.90 & 60.36 & 85.95 & 43.30 \\
\hline Detroit & 80.47 & 98.39 & 68.11 & 79.19 & 94.27 & 69.03 & 89.40 & 120.86 & 68.43 \\
\hline Hawaii & 53.33 & 69.23 & 40.51 & 63.72 & 74.88 & 54.60 & 31.83 & 33.45 & - \\
\hline Iowa & 68.97 & 89.33 & 54.17 & 68.68 & 88.80 & 54.08 & 106.25 & 152.93 & 68.16 \\
\hline Kentucky & 100.56 & 131.35 & 78.18 & 100.68 & 131.31 & 78.31 & 106.47 & 143.92 & 81.40 \\
\hline Louisiana & 79.58 & 107.83 & 58.90 & 79.33 & 102.99 & 61.79 & 81.71 & 125.88 & 52.05 \\
\hline New Jersey & 65.20 & 78.30 & 56.26 & 66.61 & 78.69 & 58.46 & 66.62 & 89.50 & 51.86 \\
\hline New Mexico & 46.30 & 55.73 & 38.69 & 48.67 & 58.02 & 41.07 & 42.71 & 55.26 & 31.30 \\
\hline Seattle-Puget Sound & 67.66 & 77.15 & 60.71 & 68.94 & 77.36 & 62.89 & 71.91 & 91.12 & 56.23 \\
\hline Utah & 29.08 & 36.20 & 23.17 & 28.96 & 35.93 & 23.19 & 56.33 & 80.71 & - \\
\hline 11 SEER Areas ${ }^{b}$ & 57.37 & 69.58 & 48.48 & 58.20 & 68.77 & 50.62 & 72.30 & 97.49 & 55.26 \\
\hline 13 SEER Areas ${ }^{b}$ & 57.49 & 69.79 & 48.53 & 58.27 & 68.91 & 50.63 & 72.05 & 97.39 & 54.94 \\
\hline 17 SEER Areas ${ }^{b}$ & 62.47 & 76.25 & 52.40 & 63.78 & 76.28 & 54.71 & 73.55 & 101.18 & 54.84 \\
\hline
\end{tabular}

Rates are per 100,000 and are age-adjusted to the 2000 US Std Population (19 age groups - Census P25-1130)

The SEER 9 areas are San Francisco, Connecticut, Detroit, Hawail, Iowa, New Mexico, Seattle, Utah and Atlanta.

The SEER 11 areas comprise the SEER 9 areas plus San Jose-Monterey and Los Angeles.

The SEER 13 areas comprise the SEER 11 areas plus the Alaska Native Registry and Rural Georgia.

The SEER 17 areas comprise the SEER 13 areas plus California excluding SF/SJM/LA, Kentucky, Louisiana and New Jersey.

Statistic not shown. Rate based on less than 16 cases for the time interval. 
Table 15.23

Cancer of the Lung And Bronchus (Invasive)

Age-Adjusted SEER Death Rates

By Registry, Race and Sex

\begin{tabular}{ccc}
\multicolumn{3}{c}{ All Races } \\
Total Males Females
\end{tabular}

Total

Whites

Females

Total

Blacks

Females

\section{SEER Death Rates ${ }^{\mathrm{a}}, 2003-2007$}

\begin{tabular}{|c|c|c|c|c|c|c|c|c|c|}
\hline Atlanta \& Rural Georgia & 44.95 & 61.24 & 33.99 & 44.47 & 58.18 & 35.06 & 48.18 & 73.89 & 32.92 \\
\hline Atlanta & 44.35 & 59.83 & 34.05 & 43.56 & 56.40 & 34.92 & 48.47 & 73.78 & 33.56 \\
\hline Rural Georgia & 57.37 & 87.66 & 34.55 & 62.35 & 91.52 & 38.70 & 48.02 & 80.84 & 27.79 \\
\hline California & 41.67 & 51.38 & 34.51 & 42.96 & 51.66 & 36.57 & 56.33 & 76.75 & 41.93 \\
\hline Greater Bay Area & 38.31 & 45.77 & 33.05 & 39.60 & 44.83 & 36.13 & 60.40 & 78.72 & 47.82 \\
\hline San Francisco-Oakland & 39.66 & 47.13 & 34.40 & 40.41 & 45.03 & 37.39 & 62.26 & 80.07 & 50.08 \\
\hline San Jose-Monterey & 35.68 & 43.18 & 30.36 & 38.23 & 44.63 & 33.94 & 49.89 & 77.62 & 29.13 \\
\hline Los Angeles & 36.67 & 47.39 & 28.84 & 35.84 & 44.76 & 29.33 & 55.73 & 77.55 & 40.84 \\
\hline Greater California & 45.28 & 55.25 & 37.83 & 46.84 & 56.42 & 39.68 & 54.35 & 74.74 & 39.20 \\
\hline Connecticut & 48.03 & 59.65 & 40.10 & 48.79 & 59.89 & 41.24 & 43.56 & 64.87 & 30.09 \\
\hline Detroit & 55.98 & 71.87 & 44.97 & 54.74 & 68.84 & 45.05 & 63.44 & 88.33 & 46.94 \\
\hline Hawaii & 38.15 & 51.19 & 27.70 & 48.87 & 58.74 & 40.51 & 21.81 & - & - \\
\hline Iowa & 52.49 & 70.82 & 39.15 & 52.42 & 70.61 & 39.20 & 78.74 & 116.24 & 49.03 \\
\hline Kentucky & 76.54 & 105.22 & 56.03 & 76.66 & 105.28 & 56.13 & 81.79 & 115.22 & 59.48 \\
\hline Louisiana & 64.05 & 89.71 & 45.58 & 62.75 & 83.93 & 47.32 & 69.25 & 111.19 & 41.44 \\
\hline New Jersey & 48.53 & 61.48 & 39.70 & 49.61 & 62.12 & 41.02 & 52.36 & 73.16 & 39.51 \\
\hline New Mexico & 37.09 & 46.15 & 29.92 & 38.87 & 47.83 & 31.75 & 38.21 & 53.57 & 24.61 \\
\hline Seattle-Puget Sound & 50.79 & 59.94 & 44.07 & 52.20 & 60.91 & 45.83 & 55.07 & 67.82 & 43.32 \\
\hline Utah & 23.85 & 31.41 & 17.61 & 23.63 & 31.19 & 17.38 & 52.45 & 71.75 & - \\
\hline 9 SEER Areas ${ }^{b}$ & 45.54 & 57.39 & 36.94 & 46.34 & 57.21 & 38.47 & 55.94 & 78.36 & 41.01 \\
\hline 11 SEER Areas ${ }^{b}$ & 43.00 & 54.37 & 34.74 & 43.72 & 53.97 & 36.30 & 55.80 & 78.06 & 40.84 \\
\hline 13 SEER Areas ${ }^{b}$ & 43.16 & 54.61 & 34.83 & 43.86 & 54.19 & 36.36 & 55.65 & 78.02 & 40.62 \\
\hline 17 SEER Areas ${ }^{b}$ & 47.56 & 60.57 & 38.07 & 48.59 & 60.70 & 39.76 & 58.30 & 83.96 & 41.14 \\
\hline Total U.S. & 52.51 & 68.81 & 40.56 & 52.94 & 68.32 & 41.60 & 58.56 & 87.52 & 39.56 \\
\hline
\end{tabular}

US Mortality Files, National Center for Health Statistics, Centers for Disease Control and Prevention.

Rates are per 100,000 and are age-adjusted to the 2000 US Std Population (19 age groups - Census P25-1130).

The SEER 9 areas are San Francisco, Connecticut, Detroit, Hawail, lowa, New Mexico, Seattle, Utah and Atlanta.

The SEER 11 areas comprise the SEER 9 areas plus San Jose-Monterey and Los Angeles.

The SEER 13 areas comprise the SEER 11 areas plus the Alaska Native Registry and Rural Georgia.

The SEER 17 areas comprise the SEER 13 areas plus California excluding SF/SJM/LA, Kentucky, Louisiana and New Jersey.

Statistic not shown. Rate based on less than 16 cases for the time interval. 
Table 15.24

Cancer of the Lung and Bronchus (Invasive)

Age-Adjusted Cancer Deatha Rates By State, All Races, 2003-2007

Males and Females

\begin{tabular}{lcccc} 
State & Rate & SE & Rank & PD \\
\hline TOTAL U.S. & 52.51 & 0.06 & & \\
High Five States & & & & \\
Kentucky & $76.54^{\mathrm{b}}$ & 0.59 & $(01)$ & $45.76^{\mathrm{c}}$ \\
Tennessee & $67.66^{\mathrm{b}}$ & 0.47 & $(02)$ & $28.84^{\mathrm{c}}$ \\
West Virginia & $67.56^{\mathrm{b}}$ & 0.78 & $(03)$ & $28.66^{\mathrm{c}}$ \\
Arkansas & $67.06^{\mathrm{b}}$ & 0.66 & $(04)$ & $27.70^{\mathrm{c}}$ \\
Mississippi & $66.49^{\mathrm{b}}$ & 0.68 & $(05)$ & $26.62^{\mathrm{c}}$ \\
& & & & \\
Low Five States & & & & \\
California & $41.67^{\mathrm{b}}$ & 0.16 & $(47)$ & $-20.64^{\mathrm{c}}$ \\
Colorado & $39.05^{\mathrm{b}}$ & 0.45 & $(48)$ & $-25.64^{\mathrm{c}}$ \\
Hawaii & $38.15^{\mathrm{b}}$ & 0.74 & $(49)$ & $-27.36^{\mathrm{c}}$ \\
New Mexico & $37.09^{\mathrm{b}}$ & 0.62 & $(50)$ & $-29.37^{\mathrm{c}}$ \\
Utah & $23.85^{\mathrm{b}}$ & 0.51 & $(51)$ & $-54.58^{\mathrm{c}}$
\end{tabular}

$\begin{array}{lllrr}\text { State } & \text { Rate } & \text { SE } & \text { Rank } & \text { PD } \\ \text { Alabama } & & & & \\ \text { Alaska } & 62.75^{\mathrm{b}} & 0.51 & (08) & 19.49^{\mathrm{c}} \\ \text { Arizona } & 53.81 & 1.72 & (22) & 2.46 \\ \text { Arkansas } & 43.55^{\mathrm{b}} & 0.38 & (45) & -17.07^{\mathrm{c}} \\ \text { California } & 67.06^{\mathrm{b}} & 0.66 & (04) & 27.70^{\mathrm{c}} \\ \text { Colorado } & 41.67^{\mathrm{b}} & 0.16 & (47) & -20.64^{\mathrm{c}} \\ \text { Connecticut } & 39.05^{\mathrm{b}} & 0.45 & (48) & -25.64^{\mathrm{c}} \\ \text { Delaware } & 48.03^{\mathrm{b}} & 0.50 & (40) & -8.53^{\mathrm{c}} \\ \text { D.C. } & 61.41^{\mathrm{b}} & 1.16 & (11) & 16.95^{\mathrm{c}} \\ \text { Florida } & 48.60 & 1.31 & (36) & -7.45^{\mathrm{c}} \\ \text { Georgia } & 52.08 & 0.21 & (30) & -0.83 \\ \text { Hawaii } & 56.90^{\mathrm{b}} & 0.39 & (17) & 8.35^{\mathrm{c}} \\ \text { Idaho } & 38.15^{\mathrm{b}} & 0.74 & (49) & -27.36^{\mathrm{c}} \\ \text { Illinois } & 43.31^{\mathrm{b}} & 0.81 & (46) & -17.52^{\mathrm{c}} \\ \text { Indiana } & 54.09^{\mathrm{b}} & 0.30 & (21) & 3.01^{\mathrm{c}} \\ \text { Iowa } & 62.62^{\mathrm{b}} & 0.45 & (09) & 19.24^{\mathrm{c}} \\ \text { Kansas } & 52.49 & 0.56 & (28) & -0.04^{2} \\ \text { Kentucky } & 54.29 & 0.62 & (20) & 3.38 \\ \text { Louisiana } & 76.54^{\mathrm{b}} & 0.59 & (01) & 45.76^{\mathrm{c}} \\ \text { Maine } & 64.05^{\mathrm{b}} & 0.55 & (06) & 21.98^{\mathrm{c}} \\ \text { Maryland } & 60.45^{\mathrm{b}} & 0.88 & (12) & 15.11^{\mathrm{c}} \\ \text { Massachusetts } & 53.76 & 0.45 & (23) & 2.37 \\ \text { Michigan } & 52.47 & 0.39 & (29) & -0.08 \\ \text { Minnesota } & 55.98^{\mathrm{b}} & 0.33 & (18) & 6.60^{\mathrm{c}} \\ \text { Mississippi } & 45.78^{\mathrm{b}} & 0.43 & (42) & -12.81^{\mathrm{c}} \\ \text { Missouri } & 66.49^{\mathrm{b}} & 0.68 & (05) & 26.62^{\mathrm{c}} \\ & 62.51^{\mathrm{b}} & 0.45 & (10) & 19.04^{\mathrm{c}} \\ & & & & \end{array}$

Montana

Nebraska

Nevada

New Hampshire

New Jersey

New Mexico

New York

North Carolina

North Dakota

Ohio

Oklahoma

Oregon

Pennsylvania

Rhode Island

South Carolina

South Dakota

Tennessee

Texas

Utah

Vermont

Virginia

Washington

West Virginia

Wisconsin

Wyoming
Rate

\subsection{4}

$48.32^{\mathrm{b}}$

$57.44^{b}$

53.45

$48.53^{\mathrm{b}}$

$37.09^{\mathrm{b}}$

$45.25^{\mathrm{b}}$

$58.85^{\mathrm{b}}$

$45.06^{\mathrm{b}}$

$59.82^{\mathrm{b}}$

$63.24^{\mathrm{b}}$

53.52

53.25

53.03

$58.96^{\mathrm{b}}$

48.89

$67.66^{\mathrm{b}}$

$50.59^{\mathrm{b}}$

$23.85^{\mathrm{b}}$

50.55

$55.65^{b}$

51.62

$67.56^{\mathrm{b}}$

$48.50^{\mathrm{b}}$

$45.90^{\mathrm{b}}$

SE Rank PD

0.99

0.72

0.73

0.90

0.33

0.62

0.21

0.37

1.13

0.31

0.58

0.53

0.27

0.95

0.52

1.06

0.47

0.23

0.51

1. 22

0.39

0.42

0.78

0.41

1. 34
(32) -3.19

$\begin{array}{ll}(39) & -7.98^{\circ}\end{array}$

(25) 1.79

(37) $\quad-7.59^{\circ}$

(50) $\quad-29.37^{\mathrm{C}}$

(43) $\quad-13.83^{\mathrm{C}}$

(15) $\quad 12.07^{\mathrm{C}}$

(44) $\quad-14.20^{\circ}$

(13) $\quad 13.92^{\circ}$

$\begin{array}{ll}(13) & 13.92^{\mathrm{C}} \\ (07) & 20.42^{\mathrm{C}}\end{array}$

(24) 1.92

(26) 1.41

(27) 0.98

(14) $\quad 12.28^{\mathrm{C}}$

(02) $\quad 28.84^{\mathrm{C}}$

(33) -3.67

(51) $\quad-54.58^{c}$

(34) -3.73

(19) $\quad 5.97^{\mathrm{C}}$

(31)

(03)

(38)

$-1.69$

$28.66^{\mathrm{C}}$

$-7.63^{\mathrm{C}}$

(41)

$-12.60^{\mathrm{C}}$ $\begin{array}{ll}(16) & 9.38^{\mathrm{C}}\end{array}$

(35) $\quad-6.89^{\circ}$
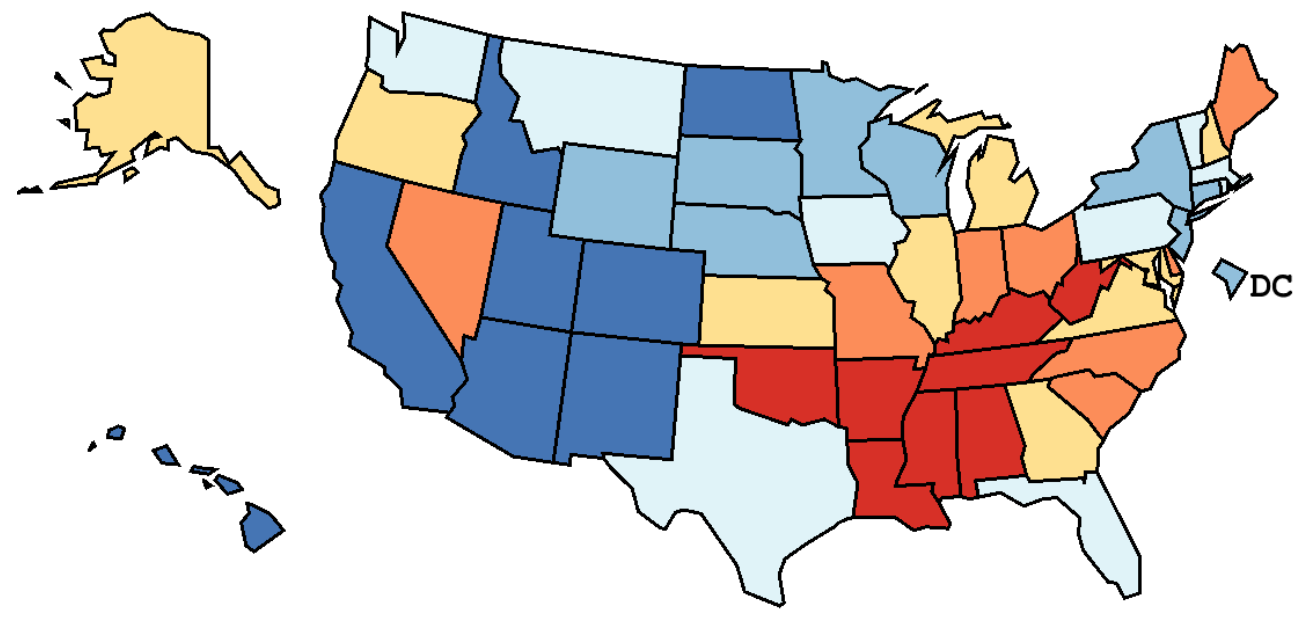

Age-Adj usted Death Rates per 100, 000 Quantile Interval

$62.63-76.54$

$56.91-62.62$

53. $26-56.90$

48.90 - 53. 25

45.07 - 48.89

$23.85-45.06$

U. S. Rate: 52. 51

\footnotetext{
a US Mortality Files, National Center for Health Statistics, Centers for Disease Control and Prevention. Rates are per 100,000 and are age-adjusted to the 2000 US Std Population (19 age groups - Census P25-1130).

Difference between state rate and total U.S. rate is statistically significant (p<=.0002) Absolute percent difference between state rate and total U.S. rate is 5\% or more.

SE Standard error of the rate.

PD Percent difference between state rate and total U.S. rate.
} 
Table 15.25

Cancer of the Lung and Bronchus (Invasive)

Age-Adjusted Cancer Death ${ }^{a}$ Rates By State, All Races, 2003-2007

Males

\begin{tabular}{lrrrc} 
State & Rate & SE & Rank & PD \\
\hline TOTAL U.S. & 68.81 & 0.10 & & \\
High Five States & & & & \\
Kentucky & $105.22^{\mathrm{b}}$ & 1.08 & $(01)$ & $52.91^{\mathrm{c}}$ \\
Mississippi & $99.40^{\mathrm{b}}$ & 1.30 & $(02)$ & $44.44^{\mathrm{c}}$ \\
Tennessee & $95.72^{\mathrm{b}}$ & 0.87 & $(03)$ & $39.11^{\mathrm{c}}$ \\
Arkansas & $93.17^{\mathrm{b}}$ & 1.19 & $(04)$ & $35.39^{\mathrm{c}}$ \\
Alabama & $92.19^{\mathrm{b}}$ & 0.96 & $(05)$ & $33.98^{\mathrm{c}}$ \\
& & & & \\
Low Five States & & & & \\
California & $51.38^{\mathrm{b}}$ & 0.28 & $(47)$ & $-25.34^{\mathrm{c}}$ \\
Hawail & $51.19^{\mathrm{b}}$ & 1.28 & $(48)$ & $-25.61^{\mathrm{c}}$ \\
Colorado & $47.82^{\mathrm{b}}$ & 0.77 & $(49)$ & $-30.50^{\mathrm{c}}$ \\
New Mexico & $46.15^{\mathrm{b}}$ & 1.05 & $(50)$ & $-32.94^{\mathrm{c}}$ \\
Utah & $31.41^{\mathrm{b}}$ & 0.88 & $(51)$ & $-54.35^{\mathrm{c}}$
\end{tabular}

\begin{tabular}{|c|c|c|c|c|}
\hline State & Rate & $\mathrm{SE}$ & Rank & $\mathrm{PD}$ \\
\hline Alabama & $92.19^{b}$ & 0.96 & (05) & $33.98^{c}$ \\
\hline Alaska & 64.83 & 2.83 & (33) & $-5.79^{c}$ \\
\hline Arizona & $54.11^{\mathrm{b}}$ & 0.63 & (45) & $-21 \cdot 36^{c}$ \\
\hline Arkansas & $93.17^{b}$ & 1.19 & $(04)$ & $35.39^{c}$ \\
\hline California & $51.38^{b}$ & 0.28 & $(47)$ & $-25.34^{c}$ \\
\hline Colorado & $47.82^{b}$ & 0.77 & (49) & $-30.50^{c}$ \\
\hline Connecticut & $59.65^{b}$ & 0.87 & $(40)$ & $-13 \cdot 32^{c}$ \\
\hline Delaware & $76.39^{\mathrm{b}}$ & 1.99 & (16) & $11.02^{\mathrm{C}}$ \\
\hline D.C. & 69.13 & 2.48 & (24) & 0.46 \\
\hline Florida & $66.22^{\mathrm{b}}$ & 0.36 & $(27)$ & -3.76 \\
\hline Georgia & $81.58^{\mathrm{b}}$ & 0.74 & (13) & $18.55^{\mathrm{c}}$ \\
\hline Hawaii & $51.19^{b}$ & 1.28 & (48) & $-25.61^{c}$ \\
\hline Idaho & $53.38^{b}$ & 1.34 & $(46)$ & $-22.43^{c}$ \\
\hline Illinois & $71.07^{\mathrm{b}}$ & 0.53 & (21) & 3.28 \\
\hline Indiana & $83.85^{\mathrm{b}}$ & 0.80 & (11) & $21.86^{C}$ \\
\hline Iowa & 70.82 & 0.98 & $(22)$ & 2.91 \\
\hline Kansas & 72.29 & 1.09 & (19) & $5.06^{c}$ \\
\hline Kentucky & $105.22^{b}$ & 1.08 & $(01)$ & $52.91^{C}$ \\
\hline Louisiana & $89.71^{\mathrm{b}}$ & 1.00 & (07) & $30.37^{c}$ \\
\hline Maine & $76.88^{\mathrm{b}}$ & 1.53 & (15) & $11.73^{\mathrm{C}}$ \\
\hline Maryland & 69.09 & 0.80 & (25) & 0.41 \\
\hline Massachusetts & $65.46^{b}$ & 0.68 & (31) & -4.88 \\
\hline Michigan & $72.50^{\mathrm{b}}$ & 0.58 & (18) & $5.36^{c}$ \\
\hline Minnesota & $58.28^{b}$ & 0.73 & $(42)$ & $-15 \cdot 31^{\mathrm{c}}$ \\
\hline Mississippi & $99.40^{\mathrm{b}}$ & 1.30 & $(02)$ & $44.44^{c}$ \\
\hline Missouri & $84.35^{b}$ & 0.80 & (09) & $22.57^{c}$ \\
\hline
\end{tabular}

SE Rank PD

Montana

Nebraska

Nevada

New Hampshire

New Jersey

New Mexico

New York

North Carolina

North Dakota

Ohio

Oklahoma

Oregon

Pennsylvania

Rhode Island

South Carolina

South Dakota

Tennessee

Texas

Utah

Vermont

Virginia

Washington

West Virginia

Wisconsin

Rate

SE

(39) $\quad-11.79^{\circ}$

$\begin{array}{llll}60.70 & 1.61 & (39) & -11.79^{\circ} \\ 65.63 & 1.28 & (28) & -4.63 \\ 64.96 & 1.14 & (32) & -5.60^{\circ}\end{array}$

$64.96-1.14$

65.49

$61.48^{\mathrm{b}}$

$(32)$
$(30)$

$-5.60^{\mathrm{C}}$

$\begin{array}{lll}1.57 & (38) & -10.66^{\circ}\end{array}$

$\begin{array}{llll}46.15^{\mathrm{b}} & 1.05 & (50) & -32.94^{\mathrm{c}}\end{array}$

$57.67^{\mathrm{b}}$

$82.49^{\mathrm{b}}$

$58.57^{\mathrm{b}}$

$80.32^{\mathrm{b}}$

$84.98^{\mathrm{b}}$

$64.16^{\mathrm{b}}$

$71.45^{\mathrm{b}}$

69.75

$83.92^{\mathrm{b}}$

65.55

0.37

0.68

1.92

$-16.20^{\circ}$

$\begin{array}{lr}(43) & -16.20^{C} \\ (12) & 19.87^{C}\end{array}$

$\begin{array}{lr}(12) & 19.87^{\circ} \\ (41) & -14.89^{\circ}\end{array}$

$\begin{array}{rr}(14) & -16.73^{\mathrm{C}}\end{array}$

$\begin{array}{lll}0.56 & (14) & 16.73^{\mathrm{C}} \\ 1.03 & (08) & 23.50^{\mathrm{C}} \\ 0.87 & (34) & -6.76^{\mathrm{C}}\end{array}$

$\begin{array}{lll}0.87 & (34) & -6.76^{\circ}\end{array}$

$\begin{array}{lll}0.48 & (20) & 3.83\end{array}$

1.69

0.95

1.84

$95.72^{b}$

68.26

$31.41^{\mathrm{b}}$

0.87

0.42

0.88

61.84

2.07

$74.53^{b}$

$61.51^{\mathrm{b}}$

$91.03^{b}$

0.71

0.69

1.39

$62.35^{b}$

0.71

Wyoming

$55.56^{\mathrm{b}}$

2.22

(23)

(10)

(29)

1.37

(03)

(26)

(51)

(36)

(17)

(37)

(06)

(35)

$-4.75$

$39.11^{\mathrm{C}}$

$-0.80$

$-54.35^{\mathrm{C}}$

$-10.14^{\mathrm{C}}$

$8.31^{\mathrm{C}}$

$-10.61^{\mathrm{c}}$

$32.28^{\mathrm{C}}$

(44)

$-9.39^{\mathrm{C}}$

$-19.25^{\mathrm{C}}$
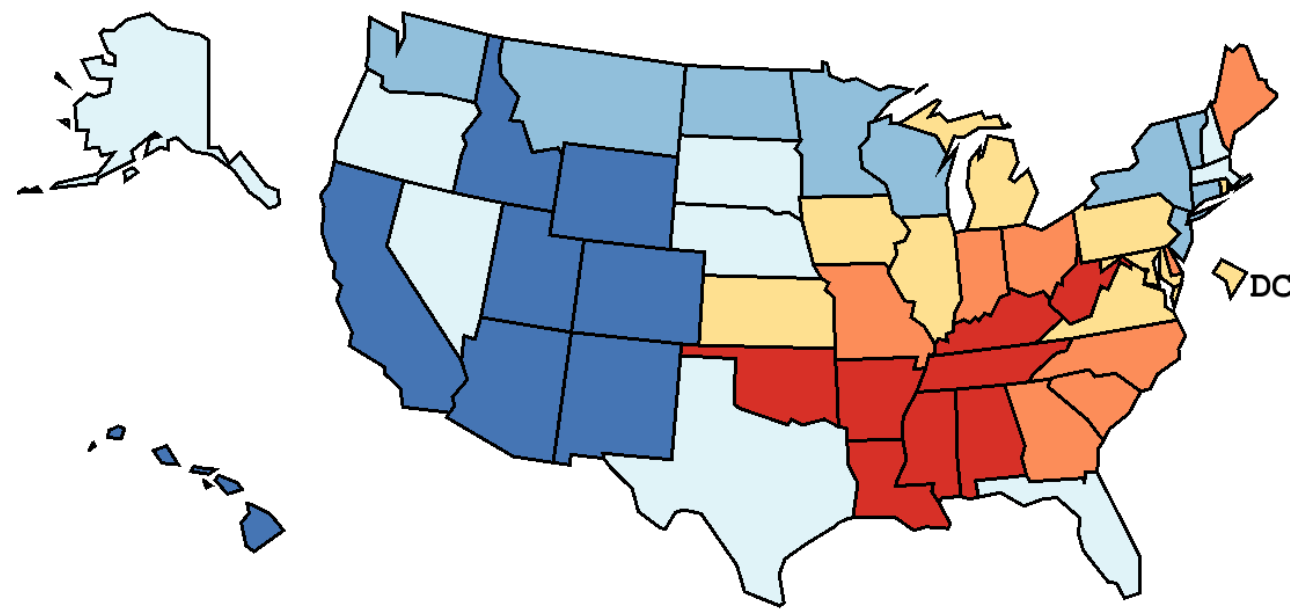

Age-Adjusted Death Rates per 100,000 Quantile Interval

84.36 - 105. 22

$74.54-84.35$

$68.27-74.53$

$62.36-68.26$

55. $57-62.35$

31.41 - 55.56

U. S. Rate: 68.81

\footnotetext{
a US Mortality Files, National Center for Health Statistics, Centers for Disease Control and Prevention. Rates are per 100,000 and are age-adjusted to the 2000 US Std Population (19 age groups - Census P25-1130).

Difference between state rate and total U.S. rate is statistically significant (p<=.0002) Absolute percent difference between state rate and total U.S. rate is $5 \%$ or more.

SE Standard error of the rate.

PD Percent difference between state rate and total U.S. rate.
} 
Table 15.26

Cancer of the Lung And Bronchus (Invasive)

Age-Adjusted Cancer Death ${ }^{2}$ Rates By State, All Races, 2003-2007

Females

\begin{tabular}{lcccc} 
State & Rate & SE & Rank & PD \\
\hline TOTAL U.S. & 40.56 & 0.07 & & \\
High Five States & & & & \\
Kentucky & $56.03^{\mathrm{b}}$ & 0.68 & $(01)$ & $38.16^{\mathrm{c}}$ \\
Nevada & $50.87^{\mathrm{b}}$ & 0.93 & $(02)$ & $25.43^{\mathrm{c}}$ \\
West Virginia & $50.47^{\mathrm{b}}$ & 0.91 & $(03)$ & $24.45^{\mathrm{c}}$ \\
Delaware & $50.37^{\mathrm{b}}$ & 1.41 & $(04)$ & $24.19^{\mathrm{c}}$ \\
Maine & $48.71^{\mathrm{b}}$ & 1.06 & $(05)$ & $20.09^{\mathrm{c}}$ \\
& & & & \\
Low Five States & & & & \\
California & $34.51^{\mathrm{b}}$ & 0.20 & $(47)$ & $-14.90^{\mathrm{c}}$ \\
Colorado & $32.85^{\mathrm{b}}$ & 0.55 & $(48)$ & $-19.00^{\mathrm{c}}$ \\
New Mexico & $29.92^{\mathrm{b}}$ & 0.75 & $(49)$ & $-26.24^{\mathrm{c}}$ \\
Hawaii & $27.70^{\mathrm{b}}$ & 0.85 & $(50)$ & $-31.70^{\mathrm{c}}$ \\
Utah & $17.61^{\mathrm{b}}$ & 0.60 & $(51)$ & $-56.57^{\mathrm{c}}$
\end{tabular}

$\begin{array}{lllrr} & & & & \\ \text { Alabama } & 41.69 & 0.55 & (27) & 2.79 \\ \text { Alaska } & 44.41 & 2.13 & (15) & 9.51^{\mathrm{c}} \\ \text { Arizona } & 34.92^{\mathrm{b}} & 0.46 & (45) & -13.89^{\mathrm{c}} \\ \text { Arkansas } & 47.46^{\mathrm{b}} & 0.75 & (08) & 17.02^{\mathrm{c}} \\ \text { California } & 34.51^{\mathrm{b}} & 0.20 & (47) & -14.90^{\mathrm{c}} \\ \text { Colorado } & 32.85^{\mathrm{b}} & 0.55 & (48) & -19.00^{\mathrm{c}} \\ \text { Connecticut } & 40.10 & 0.61 & (32) & -1.13 \\ \text { Delaware } & 50.37^{\mathrm{b}} & 1.41 & (04) & 24.19^{\mathrm{c}} \\ \text { D.C. } & 34.91^{\mathrm{b}} & 1.45 & (46) & -13.93^{\mathrm{c}} \\ \text { Florida } & 40.60 & 0.26 & (30) & 0.11^{2} \\ \text { Georgia } & 39.74 & 0.43 & (33) & -2.01^{\mathrm{c}} \\ \text { Hawaii } & 27.70^{\mathrm{b}} & 0.85 & (50) & -31.70^{\mathrm{c}} \\ \text { Idaho } & 35.44^{\mathrm{b}} & 0.99 & (43) & -12.61^{\mathrm{c}} \\ \text { Illinois } & 42.21^{\mathrm{b}} & 0.35 & (23) & 4.08 \\ \text { Indiana } & 47.61^{\mathrm{b}} & 0.52 & (06) & 17.39^{\mathrm{c}} \\ \text { Iowa } & 39.15 & 0.65 & (35) & -3.47 \\ \text { Kansas } & 41.25 & 0.73 & (28) & 1.70 \\ \text { Kentucky } & 56.03^{\mathrm{b}} & 0.68 & (01) & 38.16^{\mathrm{c}} \\ \text { Louisiana } & 45.58^{\mathrm{b}} & 0.61 & (11) & 12.37^{\mathrm{c}} \\ \text { Maine } & 48.71^{\mathrm{b}} & 1.06 & (05) & 20.09^{\mathrm{c}} \\ \text { Maryland } & 42.94^{\mathrm{b}} & 0.53 & (21) & 5.87^{\mathrm{c}} \\ \text { Massachusetts } & 43.77^{\mathrm{b}} & 0.47 & (18) & 7.92^{\mathrm{c}} \\ \text { Michigan } & 44.07^{\mathrm{b}} & 0.39 & (17) & 8.67^{\mathrm{c}} \\ \text { Minnesota } & 36.96^{\mathrm{b}} & 0.52 & (39) & -8.88^{\mathrm{c}} \\ \text { Mississippi } & 43.04 & 0.72 & (20) & 6.12^{\mathrm{c}} \\ \text { Missouri } & 46.61^{\mathrm{b}} & 0.52 & (10) & 14.93^{\mathrm{c}}\end{array}$

Montana
Nebraska
Nevada
New Hampshire
New Jersey
New Mexico
New York
North Carolina
North Dakota
Ohio
Oklahoma
Oregon
Pennsylvania
Rhode Island
South Carolina
South Dakota
Tennessee
Texas
Utah
Vermont
Virginia
Washington
West Virginia
Wisconsin
Wyoming

Rate

SE Rank $\quad$ PD

43.28

$35.61^{\mathrm{b}}$
$50.87^{\mathrm{b}}$

$50.87^{\mathrm{b}}$
$44.69^{\mathrm{b}}$

44.69
39.70

$29.92^{\mathrm{b}}$

$36.68^{\mathrm{b}}$

$42.03^{\mathrm{b}}$

$35.11^{\mathrm{b}}$

$45.34^{\mathrm{b}}$

$47.09^{\mathrm{b}}$

$45.49^{\circ}$

40.43

42.04

40.64

36.48

$47.54^{\mathrm{b}}$

$37.53^{\mathrm{b}}$

$17.61^{\mathrm{b}}$

42.45

42.11

$44.30^{\mathrm{b}}$

$50.47^{\mathrm{b}}$

$38.55^{\mathrm{b}}$

38.43

1.24
0.84
0.93
1.10
0.39
0.75
0.25
0.41
1.36
0.36
0.67
0.66
0.31
1.12
0.57
1.25
0.52
0.27
0.60
1.51
0.45
0.52
0.91
0.49
1.68

(19)

$6.71^{\mathrm{C}}$

(42) $-12.19^{\circ}$

$\begin{array}{lll}0.93 & (02) & 25.43^{\mathrm{C}} \\ .10 & (14) & 10.18^{\mathrm{C}}\end{array}$

$0.39 \quad(34) \quad-2.13$

(49) $\quad-26.24^{c}$

$\begin{array}{lr}(49) & -26.24^{c} \\ (40) & -9.57^{c}\end{array}$

$\begin{array}{rrr}0.25 & (40) & -9.57 \\ 0.41 & (26) & 3.64\end{array}$

$1.36 \quad(44) \quad-13.44^{c}$

$\begin{array}{lll}0.36 & (13) & 11.78^{C}\end{array}$

$\begin{array}{lll}0.67 & (09) & 16.10^{\mathrm{C}}\end{array}$

$\begin{array}{lll}.66 & (12) & 12.16^{\mathrm{C}}\end{array}$

(31) $\quad-0.30$

$\begin{array}{ll}(25) & 3.65\end{array}$

$\begin{array}{ll}(25) & 3.65 \\ (29) & 0.21\end{array}$

(41) $\quad-10.04^{\mathrm{c}}$

(07) $\quad 17.22^{\mathrm{C}}$

$\begin{array}{ll}(38) & -7.47^{\mathrm{C}}\end{array}$

(51) $\quad-56.57^{\mathrm{C}}$

(22) $\quad 4.68$

$\begin{array}{ll}(24) & 3.83 \\ (16) & 9.23^{\mathrm{C}}\end{array}$

$(03) \quad 24.45^{\circ}$

(36) -4.96

$6.12^{\circ}$
$4.93^{c}$

(37)

-4.96
$-5.25^{c}$
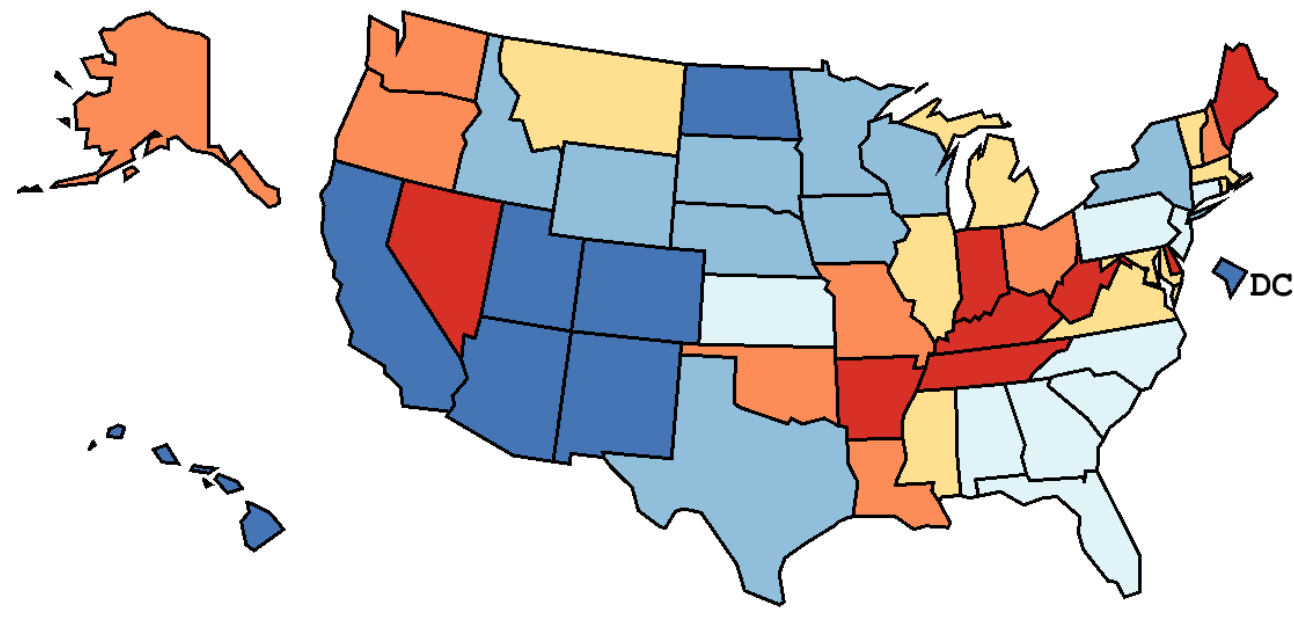

Age-Adj usted Death Rates per 100, 000 Quantile Interval
$47.10-56.03$
$44.08-47.09$
$42.04-44.07$
39. 16 - 42.03
35.12 - 39.15
$17.61-35.11$

U. S. Rate: 40.56

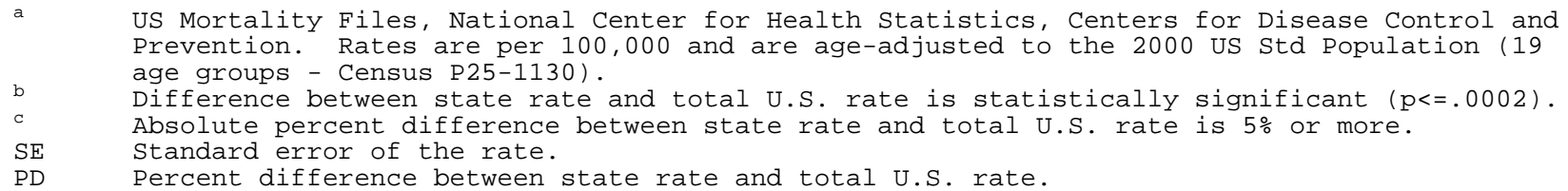




\begin{tabular}{|c|c|c|c|c|c|c|c|c|c|c|c|}
\hline Years Sinc & Diagnosis & 0 to $<5$ & 5 to $<10$ & 10 to $<15$ & 15 to $<20$ & 20 to $<25$ & 25 to $<30$ & 0 to $<17^{\mathrm{e}}$ & $0 \quad$ to $<32^{\mathrm{e}}$ & $>=32^{g}$ & Complete \\
\hline$\frac{\text { Race }}{\text { All Races }{ }^{b}}$ & $\begin{array}{l}\frac{\text { Sex }}{\text { Both Sexes }} \\
\text { Males } \\
\text { Females }\end{array}$ & $\begin{array}{l}213,542 \\
100,425 \\
113,117\end{array}$ & $\begin{array}{l}69,437 \\
30,787 \\
38,650\end{array}$ & $\begin{array}{l}38,895 \\
17,826 \\
21,069\end{array}$ & $\begin{array}{r}20,533 \\
9,540 \\
10,993\end{array}$ & $\begin{array}{r}10,999 \\
4,966 \\
6,033\end{array}$ & $\begin{array}{l}5,743 \\
2,750 \\
2,993\end{array}$ & $\begin{array}{l}331,521 \\
153,231 \\
178,290\end{array}$ & $\begin{array}{l}360,606 \\
167,066 \\
193,540\end{array}$ & $\begin{array}{r}10,011 \\
5,673 \\
4,338\end{array}$ & $\begin{array}{l}370,617 \\
172,739 \\
197,878\end{array}$ \\
\hline White ${ }^{b}$ & $\begin{array}{l}\text { Both Sexes } \\
\text { Males } \\
\text { Females }\end{array}$ & $\begin{array}{r}183,440 \\
85,221 \\
98,219\end{array}$ & $\begin{array}{l}61,075 \\
26,665 \\
34,410\end{array}$ & $\begin{array}{l}34,181 \\
15,348 \\
18,833\end{array}$ & $\begin{array}{r}18,319 \\
8,367 \\
9,952\end{array}$ & $\begin{array}{l}9,701 \\
4,347 \\
5,354\end{array}$ & $\begin{array}{l}5,177 \\
2,440 \\
2,737\end{array}$ & $\begin{array}{l}287,364 \\
130,986 \\
156,378\end{array}$ & $\begin{array}{l}313,121 \\
143,006 \\
170,115\end{array}$ & $\begin{array}{l}4,676 \\
2,240 \\
2,436\end{array}$ & $\begin{array}{l}317,797 \\
145,246 \\
172,551\end{array}$ \\
\hline $\mathrm{Black}^{\mathrm{b}}$ & $\begin{array}{l}\text { Both Sexes } \\
\text { Males } \\
\text { Females }\end{array}$ & $\begin{array}{l}22,255 \\
11,033 \\
11,222\end{array}$ & $\begin{array}{l}6,098 \\
2,890 \\
3,208\end{array}$ & $\begin{array}{l}3,559 \\
1,817 \\
1,742\end{array}$ & $\begin{array}{r}1,711 \\
927 \\
784\end{array}$ & $\begin{array}{r}1,022 \\
466 \\
556\end{array}$ & $\begin{array}{l}436 \\
230 \\
206\end{array}$ & $\begin{array}{l}32,646 \\
16,061 \\
16,585\end{array}$ & $\begin{array}{l}35,256 \\
17,500 \\
17,756\end{array}$ & $\begin{array}{l}429 \\
228 \\
201\end{array}$ & $\begin{array}{l}35,685 \\
17,728 \\
17,957\end{array}$ \\
\hline $\begin{array}{l}\text { Asian/ } \\
\text { Pacific } \\
\text { Islander }\end{array}$ & $\begin{array}{l}\text { Both Sexes } \\
\text { Males } \\
\text { Females }\end{array}$ & $\begin{array}{l}5,962 \\
3,109 \\
2,853\end{array}$ & $\begin{array}{r}1,642 \\
854 \\
788\end{array}$ & $\begin{array}{l}760 \\
428 \\
332\end{array}$ & $\begin{array}{l}+ \\
+ \\
+\end{array}$ & $\begin{array}{l}+ \\
+ \\
+\end{array}$ & $\begin{array}{l}+ \\
+ \\
+\end{array}$ & $\begin{array}{l}8,553 \\
4,504 \\
4,049\end{array}$ & $\begin{array}{l}+ \\
+ \\
+\end{array}$ & $\begin{array}{l}+ \\
+ \\
+\end{array}$ & $\begin{array}{l}+ \\
+ \\
+\end{array}$ \\
\hline Hispanic ${ }^{d}$ & $\begin{array}{l}\text { Both Sexes } \\
\text { Males } \\
\text { Females }\end{array}$ & $\begin{array}{l}6,506 \\
3,341 \\
3,165\end{array}$ & $\begin{array}{r}1,898 \\
929 \\
969\end{array}$ & $\begin{array}{r}1,016 \\
508 \\
508\end{array}$ & $\begin{array}{l}+ \\
+ \\
+\end{array}$ & $\begin{array}{l}+ \\
+ \\
+\end{array}$ & $\begin{array}{l}+ \\
+ \\
+\end{array}$ & $\begin{array}{l}9,674 \\
4,879 \\
4,795\end{array}$ & $\begin{array}{l}+ \\
+ \\
+\end{array}$ & $\begin{array}{l}+ \\
+ \\
+\end{array}$ & $\begin{array}{l}+ \\
+ \\
+\end{array}$ \\
\hline
\end{tabular}
Estimated prevalence percent ${ }^{a}$ on January 1, 2007, of the SEER 11 population diagnosed in the previous 17 years
By Age at Prevalence, Race/Ethnicity and Sex

\begin{tabular}{|c|c|c|c|c|c|c|c|c|c|c|c|c|}
\hline \multirow{2}{*}{\multicolumn{2}{|c|}{ Age at Prevalence }} & \multicolumn{10}{|c|}{ Age Specific (Crude) } & \multirow{2}{*}{$\frac{\text { Age-Adjusted }}{\text { All Ages }}$} \\
\hline & & All Ages & $0-9$ & $10-19$ & $20-29$ & $30-39$ & $40-49$ & $50-59$ & $60-69$ & $70-79$ & $80+$ & \\
\hline$\frac{\text { Race }}{\text { All Races }}$ & $\begin{array}{l}\frac{\text { Sex }}{\text { Both Sexes }} \\
\text { Males } \\
\text { Females }\end{array}$ & $\begin{array}{l}0.0917 \% \\
0.0867 \% \\
0.0967 \%\end{array}$ & $\begin{array}{l}0.0001 \% \\
- \\
-\end{array}$ & $\begin{array}{l}0.0003 \% \\
0.0003 \% \\
0.0002 \%\end{array}$ & $\begin{array}{l}0.0015 \% \\
0.0014 \% \\
0.0015 \%\end{array}$ & $\begin{array}{l}0.0051 \% \\
0.0049 \% \\
0.0053 \%\end{array}$ & $\begin{array}{l}0.0256 \% \\
0.0225 \% \\
0.0287 \%\end{array}$ & $\begin{array}{l}0.1080 \% \\
0.1067 \% \\
0.1093 \%\end{array}$ & $\begin{array}{l}0.3420 \% \\
0.3527 \% \\
0.3323 \%\end{array}$ & $\begin{array}{l}0.6224 \% \\
0.6718 \% \\
0.5839 \%\end{array}$ & $\begin{array}{l}0.5033 \% \\
0.5928 \% \\
0.4534 \%\end{array}$ & $\begin{array}{l}0.0958 \% \\
0.1017 \% \\
0.0918 \%\end{array}$ \\
\hline White ${ }^{c}$ & $\begin{array}{l}\text { Both Sexes } \\
\text { Males } \\
\text { Females }\end{array}$ & $\begin{array}{l}0.0983 \% \\
0.0901 \% \\
0.1064 \%\end{array}$ & $\begin{array}{l}0.0001 \% \\
- \\
-\end{array}$ & $\begin{array}{l}0.0003 \% \\
0.0003 \% \\
-\end{array}$ & $\begin{array}{l}0.0017 \% \\
0.0016 \% \\
0.0017 \%\end{array}$ & $\begin{array}{l}0.0053 \% \\
0.0049 \% \\
0.0058 \%\end{array}$ & $\begin{array}{l}0.0252 \% \\
0.0212 \% \\
0.0293 \%\end{array}$ & $\begin{array}{l}0.1053 \% \\
0.1017 \% \\
0.1089 \%\end{array}$ & $\begin{array}{l}0.3507 \% \\
0.3476 \% \\
0.3536 \%\end{array}$ & $\begin{array}{l}0.6452 \% \\
0.6773 \% \\
0.6196 \%\end{array}$ & $\begin{array}{l}0.5172 \% \\
0.5955 \% \\
0.4738 \%\end{array}$ & $\begin{array}{l}0.0980 \% \\
0.1010 \% \\
0.0964 \%\end{array}$ \\
\hline $\mathrm{Black}^{\mathrm{C}}$ & $\begin{array}{l}\text { Both Sexes } \\
\text { Males } \\
\text { Females }\end{array}$ & $\begin{array}{l}0.0781 \% \\
0.0808 \% \\
0.0757 \%\end{array}$ & $\begin{array}{l}- \\
- \\
-\end{array}$ & $\begin{array}{l}- \\
- \\
-\end{array}$ & $\begin{array}{l}0.0009 \% \\
- \\
-\end{array}$ & $\begin{array}{l}0.0055 \% \\
0.0059 \% \\
0.0051 \%\end{array}$ & $\begin{array}{l}0.0349 \% \\
0.0339 \% \\
0.0358 \%\end{array}$ & $\begin{array}{l}0.1631 \% \\
0.1773 \% \\
0.1513 \%\end{array}$ & $\begin{array}{l}0.3971 \% \\
0.4842 \% \\
0.3296 \%\end{array}$ & $\begin{array}{l}0.6395 \% \\
0.7644 \% \\
0.5558 \%\end{array}$ & $\begin{array}{l}0.4228 \% \\
0.5531 \% \\
0.3595 \%\end{array}$ & $\begin{array}{l}0.1054 \% \\
0.1244 \% \\
0.0924 \%\end{array}$ \\
\hline $\begin{array}{l}\text { Asian/ } \\
\text { Pacific } \\
\text { Islander }\end{array}$ & $\begin{array}{l}\text { Both Sexes } \\
\text { Males } \\
\text { Females }\end{array}$ & $\begin{array}{l}0.0695 \% \\
0.0750 \% \\
0.0642 \%\end{array}$ & $\begin{array}{l}- \\
- \\
-\end{array}$ & $\begin{array}{l}- \\
- \\
-\end{array}$ & $\begin{array}{l}- \\
- \\
-\end{array}$ & $\begin{array}{l}0.0039 \% \\
0.0042 \% \\
0.0037 \%\end{array}$ & $\begin{array}{l}0.0204 \% \\
0.0213 \% \\
0.0196 \%\end{array}$ & $\begin{array}{l}0.0804 \% \\
0.0836 \% \\
0.0776 \%\end{array}$ & $\begin{array}{l}0.2483 \% \\
0.3000 \% \\
0.2043 \%\end{array}$ & $\begin{array}{l}0.4793 \% \\
0.5872 \% \\
0.4000 \%\end{array}$ & $\begin{array}{l}0.4449 \% \\
0.5900 \% \\
0.3510 \%\end{array}$ & $\begin{array}{l}0.0741 \% \\
0.0897 \% \\
0.0625 \%\end{array}$ \\
\hline Hispanic ${ }^{d}$ & $\begin{array}{l}\text { Both Sexes } \\
\text { Males } \\
\text { Females }\end{array}$ & $\begin{array}{l}0.0211 \% \\
0.0205 \% \\
0.0217 \%\end{array}$ & $\begin{array}{l}- \\
- \\
-\end{array}$ & $\begin{array}{l}- \\
- \\
-\end{array}$ & $\begin{array}{l}0.0009 \% \\
0.0008 \% \\
0.0010 \%\end{array}$ & $\begin{array}{l}0.0031 \% \\
0.0033 \% \\
0.0027 \%\end{array}$ & $\begin{array}{l}0.0109 \% \\
0.0108 \% \\
0.0110 \%\end{array}$ & $\begin{array}{l}0.0430 \% \\
0.0452 \% \\
0.0409 \%\end{array}$ & $\begin{array}{l}0.1323 \% \\
0.1357 \% \\
0.1293 \%\end{array}$ & $\begin{array}{l}0.2727 \% \\
0.3320 \% \\
0.2293 \%\end{array}$ & $\begin{array}{l}0.2656 \% \\
0.3529 \% \\
0.2148 \%\end{array}$ & $\begin{array}{l}0.0419 \% \\
0.0488 \% \\
0.0372 \%\end{array}$ \\
\hline
\end{tabular}

US 2007 cancer prevalence counts are based on 2007 cancer prevalence proportions from the SEER registries and $1 / 1 / 2007$ US population estimates based on the average of 2006 and 2007 population estimates from the US Bureau of the Census. Statistics based on (b) SEER 9 Areas (c) SEER 11 Areas and Rural Georgia (d) NHIA for Hispanic for SEER 11 Areas and Rural Georgia.

Maximum limited-duration prevalence: 32 years for 1975-2007 SEER 9 data; 17 years for 1990-2007 SEER 11 data (used to calculate prevalence for Hispanics and Asian Pacific Islanders).

hi Percentages are age-adjusted to the 2000 US Standard Population (19 age groups - Census P25-1130) by 5-year age groups.

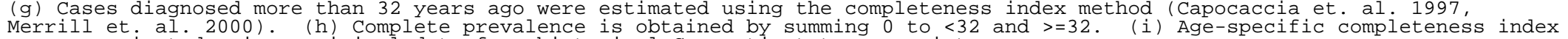
was approximated using empirical data from historical Connecticut tumor registry.

Not available. 
Cancer of the Lung and Bronchus (Invasive)

Percent Distribution and Counts by Histology among Histologically Confirmed Cases, 2003-2007 Both Sexes by Race

\begin{tabular}{|c|c|c|c|c|c|c|c|c|c|c|c|c|}
\hline \multirow[b]{2}{*}{$\underline{\text { Histology }}^{a}$} & \multicolumn{2}{|c|}{ All Races } & \multicolumn{2}{|c|}{ White } & \multicolumn{2}{|c|}{ Black } & \multicolumn{2}{|c|}{$\begin{array}{c}\text { Asian/Pacific } \\
\text { Islander }\end{array}$} & \multicolumn{2}{|c|}{$\begin{array}{c}\text { American Indian/ } \\
\text { Alaska Native }\end{array}$} & \multicolumn{2}{|c|}{ Hispanic ${ }^{c}$} \\
\hline & Count & Percent & Count & Percent & Count & Percent & Count & Percent & Count & Percent & Count & Percent \\
\hline Carcinoma & 198,736 & $98.9 \%$ & 166,400 & $98.9 \%$ & 20,038 & $99.0 \%$ & 10,881 & $99.1 \%$ & 690 & $98.0 \%$ & 10,526 & $98.7 \%$ \\
\hline Small cell (8041-8045) & 27,826 & $13.9 \%$ & 24,554 & $14.6 \%$ & 2,167 & $10.7 \%$ & 889 & $8.1 \%$ & 135 & $19.2 \%$ & 1,275 & $12.0 \%$ \\
\hline Non-small cell ${ }^{d}$ & 170,910 & $85.1 \%$ & 141,846 & $84.3 \%$ & 17,871 & $88.3 \%$ & 9,992 & $91.0 \%$ & 555 & $78.8 \%$ & 9,251 & $86.7 \%$ \\
\hline $\begin{array}{l}\text { Squamous and } \\
\text { transitional cell } \\
(8051-8052,8070-8084 \\
8120-8131)\end{array}$ & 39,682 & $19.8 \%$ & 33,213 & $19.7 \%$ & 4,485 & $22.2 \%$ & 1,668 & $15.2 \%$ & 180 & $25.6 \%$ & 1,837 & $17.2 \%$ \\
\hline $\begin{array}{l}\text { Adenocarcinoma } \\
\qquad \begin{array}{l}(8050,8140-8149, \\
8160-8162,8190-8221, \\
8250-8263,8270-8280, \\
8290-8337,8350-8390, \\
8400-8560,8570-8576, \\
8940-8941)\end{array}\end{array}$ & 75,367 & $37.5 \%$ & 62,305 & $37.0 \%$ & 7,249 & $35.8 \%$ & 5,374 & $48.9 \%$ & 161 & $22.9 \%$ & 4,276 & $40.1 \%$ \\
\hline Large cell (8011-8015) & 6,544 & $3.3 \%$ & 5,356 & $3.2 \%$ & 807 & $4.0 \%$ & 335 & $3.0 \%$ & 22 & $3.1 \%$ & 363 & $3.4 \%$ \\
\hline Other non-small cell ${ }^{e}$ & 49,317 & $24.5 \%$ & 40,972 & $24.4 \%$ & 5,330 & $26.3 \%$ & 2,615 & $23.8 \%$ & 192 & $27.3 \%$ & 2,775 & $26.0 \%$ \\
\hline $\begin{array}{l}\text { Sarcoma } \\
\quad \begin{array}{r}(8680-8713,8800-8921, \\
8990-8991,9040-9044, \\
9120-9136,9150-9252, \\
9370-9373,9540-9582)\end{array}\end{array}$ & 355 & $0.2 \%$ & 302 & $0.2 \%$ & 27 & $0.1 \%$ & 17 & $0.2 \%$ & - & - & 29 & $0.3 \%$ \\
\hline $\begin{array}{l}\text { Other specified types }{ }^{f} \\
\quad(8720-8790,8930-8936, \\
8950-8983,9000-9030, \\
9060-9110,9260-9365, \\
9380-9539)\end{array}$ & 144 & $0.1 \%$ & 129 & $0.1 \%$ & - & - & - & - & - & - & - & - \\
\hline Unspecified (8000-8005) & 1,674 & $0.8 \%$ & 1,414 & $0.8 \%$ & 163 & $0.8 \%$ & 83 & $0.8 \%$ & - & - & 101 & $0.9 \%$ \\
\hline Total & 200,909 & $100.0 \%$ & 168,245 & $100.0 \%$ & 20,239 & $100.0 \%$ & 10,985 & $100.0 \%$ & 704 & $100.0 \%$ & 10,666 & $100.0 \%$ \\
\hline
\end{tabular}

Source: SEER 17 areas (San Francisco, Connecticut, Detroit, Hawaii, Iowa, New Mexico, Seattle, Utah, Atlanta, San Jose-Monterey, Los Angeles, Alaska Native Registry, Rural Georgia, California excluding SF/SJM/LA, Kentucky, Louisiana and New Jersey). Percents may not sum to 100 due to rounding.

Excludes Kaposi Sarcoma, mesothelioma, lymphomas, leukemias, myelomas, lymphoreticular, and immunoproliferative diseases. Estimates for American Indian/Alaska Native are based on the CHSDA(Contract Health Service Delivery Area) counties. Hispanic is not mutually exclusive from whites, blacks, Asian/Pacific Islanders, and American Indians/Alaska Natives. Underlying incidence data for Hispanics are based on NHIA and exclude cases from the Alaska Native Registry. Non-small cell includes histologies 8010-8015, 8020-8022, 8030-8040, 8046, 8050-8052, 8070-8084, 8090-8110, 8120-8131, $8140-8156,8160-8162,8170-8175,8180,8190-8221,8230-8231,8240-8263,8270-8280,8290-8337,8340-8347,8350-8390,8400-8562$, $8570-8576,8580-8671,8940-8941$.

Includes large cell carcinoma, NOS, other specified carcinomas, and unspecified carcinomas (8010, 8020-8022, 8030-8040, 8046, 8090-8110, 8150-8156, 8170-8175, 8180, 8230-8231, 8240-8249,8340-8347,8561-8562, 8580-8671).

Other specific types includes melanomas.

Statistic not shown due to fewer than 16 cases during the time period. 
Cancer of the Lung and Bronchus (Invasive)

Percent Distribution and Counts by Histology among Histologically Confirmed Cases, 2003-2007 Males by Race

\begin{tabular}{|c|c|c|c|c|c|c|c|c|c|c|c|c|}
\hline \multirow[b]{2}{*}{ Histology $^{a}$} & \multicolumn{2}{|c|}{ All Races } & \multicolumn{2}{|c|}{ White } & \multicolumn{2}{|c|}{ Black } & \multicolumn{2}{|c|}{$\begin{array}{c}\text { Asian/Pacific } \\
\text { Islander } \\
\end{array}$} & \multicolumn{2}{|c|}{$\begin{array}{l}\text { American Indian/ } \\
\text { Alaska Native }\end{array}$} & \multicolumn{2}{|c|}{ Hispanic ${ }^{c}$} \\
\hline & Count & Percent & Count & Percent & Count & Percent & Count & Percent & Count & Percent & Count & Percent \\
\hline Carcinoma & 106,152 & $98.9 \%$ & 87,652 & $98.9 \%$ & 11,338 & $99.1 \%$ & 6,421 & $99.0 \%$ & 355 & $97.5 \%$ & 5,810 & $98.8 \%$ \\
\hline Small cell (8041-8045) & 13,947 & $13.0 \%$ & 12,119 & $13.7 \%$ & 1,122 & $9.8 \%$ & 608 & $9.4 \%$ & 61 & $16.8 \%$ & 706 & $12.0 \%$ \\
\hline Non-small cell ${ }^{d}$ & 92,205 & $85.9 \%$ & 75,533 & $85.2 \%$ & 10,216 & $89.3 \%$ & 5,813 & $89.6 \%$ & 294 & $80.8 \%$ & 5,104 & $86.8 \%$ \\
\hline $\begin{array}{l}\text { Squamous and } \\
\text { transitional cell } \\
(8051-8052,8070-8084 \\
8120-8131)\end{array}$ & 25,050 & $23.3 \%$ & 20,702 & $23.4 \%$ & 2,893 & $25.3 \%$ & 1,250 & $19.3 \%$ & 106 & $29.1 \%$ & 1,221 & $20.8 \%$ \\
\hline $\begin{array}{l}\text { Adenocarcinoma } \\
\qquad \begin{array}{l}(8050,8140-8149, \\
8160-8162,8190-8221, \\
8250-8263,8270-8280, \\
8290-8337,8350-8390, \\
8400-8560,8570-8576, \\
8940-8941)\end{array}\end{array}$ & 36,582 & $34.1 \%$ & 29,899 & $33.7 \%$ & 3,756 & $32.8 \%$ & 2,728 & $42.1 \%$ & 73 & $20.1 \%$ & 2,106 & $35.8 \%$ \\
\hline Large cell (8011-8015) & 3,782 & $3.5 \%$ & 3,064 & $3.5 \%$ & 473 & $4.1 \%$ & 222 & $3.4 \%$ & - & - & 228 & $3.9 \%$ \\
\hline Other non-small celle & 26,791 & $25.0 \%$ & 21,868 & $24.7 \%$ & 3,094 & $27.0 \%$ & 1,613 & $24.9 \%$ & 105 & $28.8 \%$ & 1,549 & $26.3 \%$ \\
\hline $\begin{array}{l}\text { Sarcoma } \\
\quad(8680-8713,8800-8921, \\
8990-8991,9040-9044, \\
9120-9136,9150-9252, \\
9370-9373,9540-9582)\end{array}$ & 200 & $0.2 \%$ & 168 & $0.2 \%$ & 16 & $0.1 \%$ & - & - & - & - & - & - \\
\hline $\begin{array}{l}\text { Other specified types }{ }^{f} \\
(8720-8790,8930-8936, \\
8950-8983,9000-9030, \\
9060-9110,9260-9365, \\
9380-9539)\end{array}$ & 74 & $0.1 \%$ & 69 & $0.1 \%$ & - & - & - & - & - & - & - & - \\
\hline Unspecified (8000-8005) & 881 & $0.8 \%$ & 730 & $0.8 \%$ & 89 & $0.8 \%$ & 52 & $0.8 \%$ & - & - & 51 & $0.9 \%$ \\
\hline Total & 107,307 & $100.0 \%$ & 88,619 & $100.0 \%$ & 11,445 & $100.0 \%$ & 6,486 & $100.0 \%$ & 364 & $100.0 \%$ & 5,879 & $100.0 \%$ \\
\hline
\end{tabular}

Source: SEER 17 areas (San Francisco, Connecticut, Detroit, Hawaii, Iowa, New Mexico, Seattle, Utah, Atlanta, San Jose-Monterey, Los Angeles, Alaska Native Registry, Rural Georgia, California excluding SF/SJM/LA, Kentucky, Louisiana and New Jersey). Percents may not sum to 100 due to rounding.

Excludes Kaposi Sarcoma, mesothelioma, lymphomas, leukemias, myelomas, lymphoreticular, and immunoproliferative diseases. Estimates for American Indian/Alaska Native are based on the CHSDA(Contract Health Service Delivery Area) counties. Hispanic is not mutually exclusive from whites, blacks, Asian/Pacific Islanders, and American Indians/Alaska Natives. Underlying incidence data for Hispanics are based on NHIA and exclude cases from the Alaska Native Registry. Non-small cell includes histologies 8010-8015, 8020-8022, 8030-8040, 8046, 8050-8052, 8070-8084, 8090-8110, 8120-8131, $8140-8156,8160-8162,8170-8175,8180,8190-8221,8230-8231,8240-8263,8270-8280,8290-8337,8340-8347,8350-8390,8400-8562$, $8570-8576,8580-8671,8940-8941$.

Includes large cell carcinoma, NOS, other specified carcinomas, and unspecified carcinomas (8010, 8020-8022, 8030-8040, 8046, 8090-8110, 8150-8156, 8170-8175, 8180, 8230-8231, 8240-8249,8340-8347,8561-8562, 8580-8671).

Other specific types includes melanomas.

Statistic not shown due to fewer than 16 cases during the time period. 
Percent Distribution and Counts by Histology among Histologically Confirmed Cases, 2003-2007 Females by Race

\begin{tabular}{|c|c|c|c|c|c|c|c|c|c|c|c|c|}
\hline \multirow[b]{2}{*}{ Histology $^{a}$} & \multicolumn{2}{|c|}{ All Races } & \multicolumn{2}{|c|}{ White } & \multicolumn{2}{|c|}{ Black } & \multicolumn{2}{|c|}{$\begin{array}{c}\text { Asian/Pacific } \\
\text { Islander }\end{array}$} & \multicolumn{2}{|c|}{$\begin{array}{l}\text { American Indian/ } \\
\text { Alaska Native }\end{array}$} & \multicolumn{2}{|c|}{ Hispanic ${ }^{c}$} \\
\hline & Count & Percent & Count & Percent & Count & Percent & Count & Percent & Count & Percent & Count & Percent \\
\hline Carcinoma & 92,584 & $98.9 \%$ & 78,748 & $98.9 \%$ & 8,700 & $98.9 \%$ & 4,460 & $99.1 \%$ & 335 & $98.5 \%$ & 4,716 & $98.5 \%$ \\
\hline Small cell (8041-8045) & 13,879 & $14.8 \%$ & 12,435 & $15.6 \%$ & 1,045 & $11.9 \%$ & 281 & $6.2 \%$ & 74 & $21.8 \%$ & 569 & $11.9 \%$ \\
\hline Non-small cell ${ }^{d}$ & 78,705 & $84.1 \%$ & 66,313 & $83.3 \%$ & 7,655 & $87.0 \%$ & 4,179 & $92.9 \%$ & 261 & $76.8 \%$ & 4,147 & $86.6 \%$ \\
\hline $\begin{array}{l}\text { Squamous and } \\
\text { transitional cell } \\
(8051-8052,8070-8084 \\
8120-8131)\end{array}$ & 14,632 & $15.6 \%$ & 12,511 & $15.7 \%$ & 1,592 & $18.1 \%$ & 418 & $9.3 \%$ & 74 & $21.8 \%$ & 616 & $12.9 \%$ \\
\hline $\begin{array}{l}\text { Adenocarcinoma } \\
\qquad \begin{array}{l}(8050,8140-8149, \\
8160-8162,8190-8221, \\
8250-8263,8270-8280, \\
8290-8337,8350-8390, \\
8400-8560,8570-8576, \\
8940-8941)\end{array}\end{array}$ & 38,785 & $41.4 \%$ & 32,406 & $40.7 \%$ & 3,493 & $39.7 \%$ & 2,646 & $58.8 \%$ & 88 & $25.9 \%$ & 2,170 & $45.3 \%$ \\
\hline Large cell (8011-8015) & 2,762 & $3.0 \%$ & 2,292 & $2.9 \%$ & 334 & $3.8 \%$ & 113 & $2.5 \%$ & - & - & 135 & $2.8 \%$ \\
\hline Other non-small celle & 22,526 & $24.1 \%$ & 19,104 & $24.0 \%$ & 2,236 & $25.4 \%$ & 1,002 & $22.3 \%$ & 87 & $25.6 \%$ & 1,226 & $25.6 \%$ \\
\hline $\begin{array}{l}\text { Sarcoma } \\
\quad(8680-8713,8800-8921, \\
8990-8991,9040-9044, \\
9120-9136,9150-9252, \\
9370-9373,9540-9582)\end{array}$ & 155 & $0.2 \%$ & 134 & $0.2 \%$ & - & - & - & - & - & - & - & - \\
\hline $\begin{array}{l}\text { Other specified types }{ }^{f} \\
\quad(8720-8790,8930-8936, \\
8950-8983,9000-9030, \\
9060-9110,9260-9365, \\
9380-9539)\end{array}$ & 70 & $0.1 \%$ & 60 & $0.1 \%$ & - & - & - & - & - & - & - & - \\
\hline Unspecified (8000-8005) & 793 & $0.8 \%$ & 684 & $0.9 \%$ & 74 & $0.8 \%$ & 31 & $0.7 \%$ & - & - & 50 & $1.0 \%$ \\
\hline Total & 93,602 & $100.0 \%$ & 79,626 & $100.0 \%$ & 8,794 & $100.0 \%$ & 4,499 & $100.0 \%$ & 340 & $100.0 \%$ & 4,787 & $100.0 \%$ \\
\hline
\end{tabular}

Source: SEER 17 areas (San Francisco, Connecticut, Detroit, Hawaii, Iowa, New Mexico, Seattle, Utah, Atlanta, San Jose-Monterey, Los Angeles, Alaska Native Registry, Rural Georgia, California excluding SF/SJM/LA, Kentucky, Louisiana and New Jersey). Percents may not sum to 100 due to rounding.

Excludes Kaposi Sarcoma, mesothelioma, lymphomas, leukemias, myelomas, lymphoreticular, and immunoproliferative diseases. Estimates for American Indian/Alaska Native are based on the CHSDA(Contract Health Service Delivery Area) counties. Hispanic is not mutually exclusive from whites, blacks, Asian/Pacific Islanders, and American Indians/Alaska Natives. Underlying incidence data for Hispanics are based on NHIA and exclude cases from the Alaska Native Registry. Non-small cell includes histologies 8010-8015, 8020-8022, 8030-8040, 8046, 8050-8052, 8070-8084, 8090-8110, 8120-8131, $8140-8156,8160-8162,8170-8175,8180,8190-8221,8230-8231,8240-8263,8270-8280,8290-8337,8340-8347,8350-8390,8400-8562$, $8570-8576,8580-8671,8940-8941$.

Includes large cell carcinoma, NOS, other specified carcinomas, and unspecified carcinomas (8010, 8020-8022, 8030-8040, 8046, 8090-8110, 8150-8156, 8170-8175, 8180, 8230-8231, 8240-8249,8340-8347,8561-8562, 8580-8671).

Other specific types includes melanomas.

Statistic not shown due to fewer than 16 cases during the time period. 


\section{SEER Observed Incidence, SEER Delay Adjusted Incidence and US Death Rates ${ }^{\mathrm{a}}$ Cancer of the Lung and Bronchus, by Race and Sex}

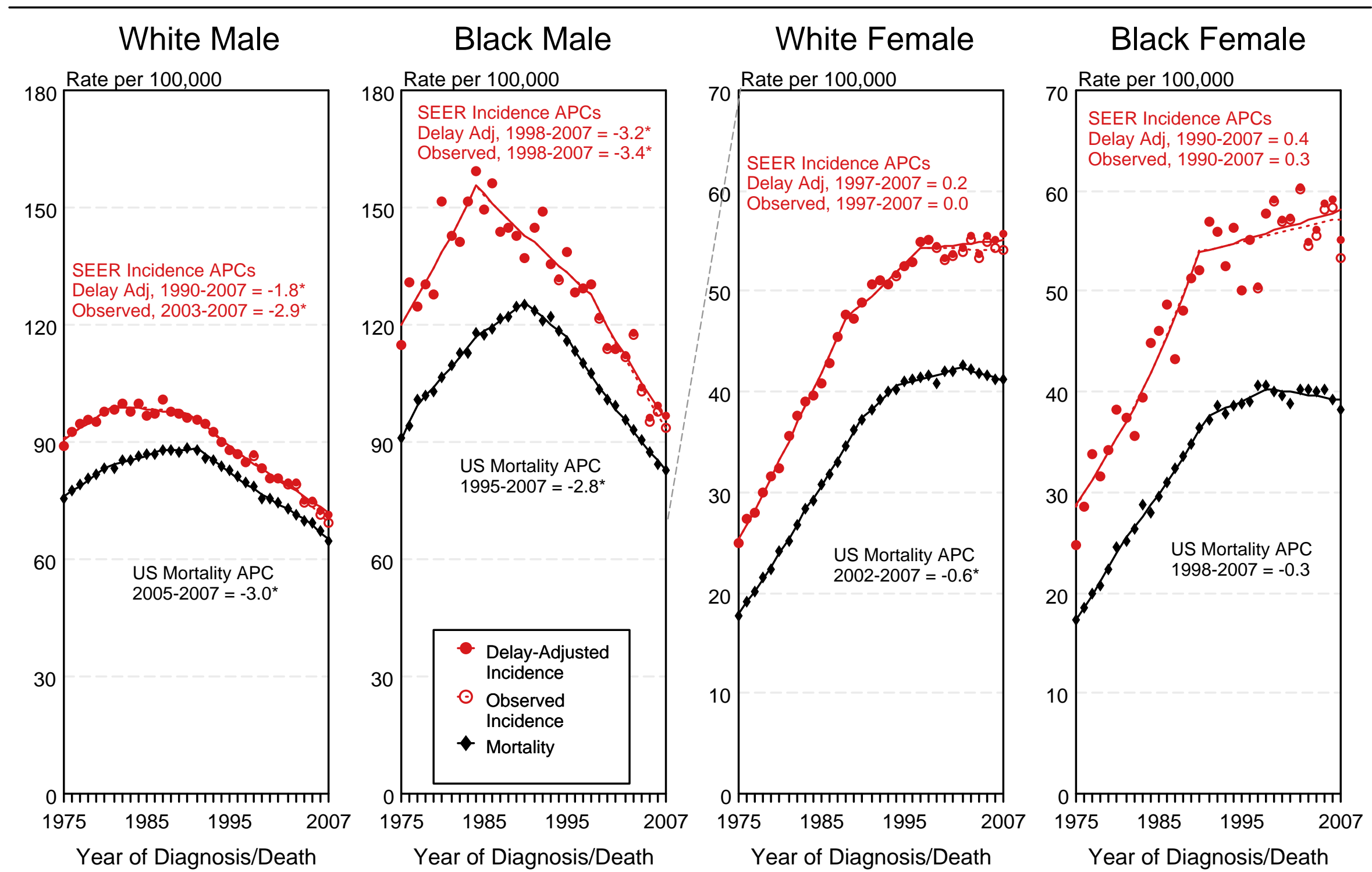

a Source: SEER 9 areas and US Mortality Files (National Center for Health Statistics, CDC).

Rates are age-adjusted to the 2000 US Std Population (19 age groups - Census P25-1103).

Regression lines and APCs are calculated using the Joinpoint Regression Program Version 3.4.3, April 2010, National Cancer Institute.

The APC is the Annual Percent Change for the regression line segments. The APC shown on the graph is for the most recent trend.

* The APC is significantly different from zero $(p<0.05)$ 
SEER Observed Incidence, SEER Delay Adjusted Incidence and US Death Rates ${ }^{\mathrm{a}}$ Cancer of the Lung and Bronchus, Under 65 Years of Age, by Race and Sex

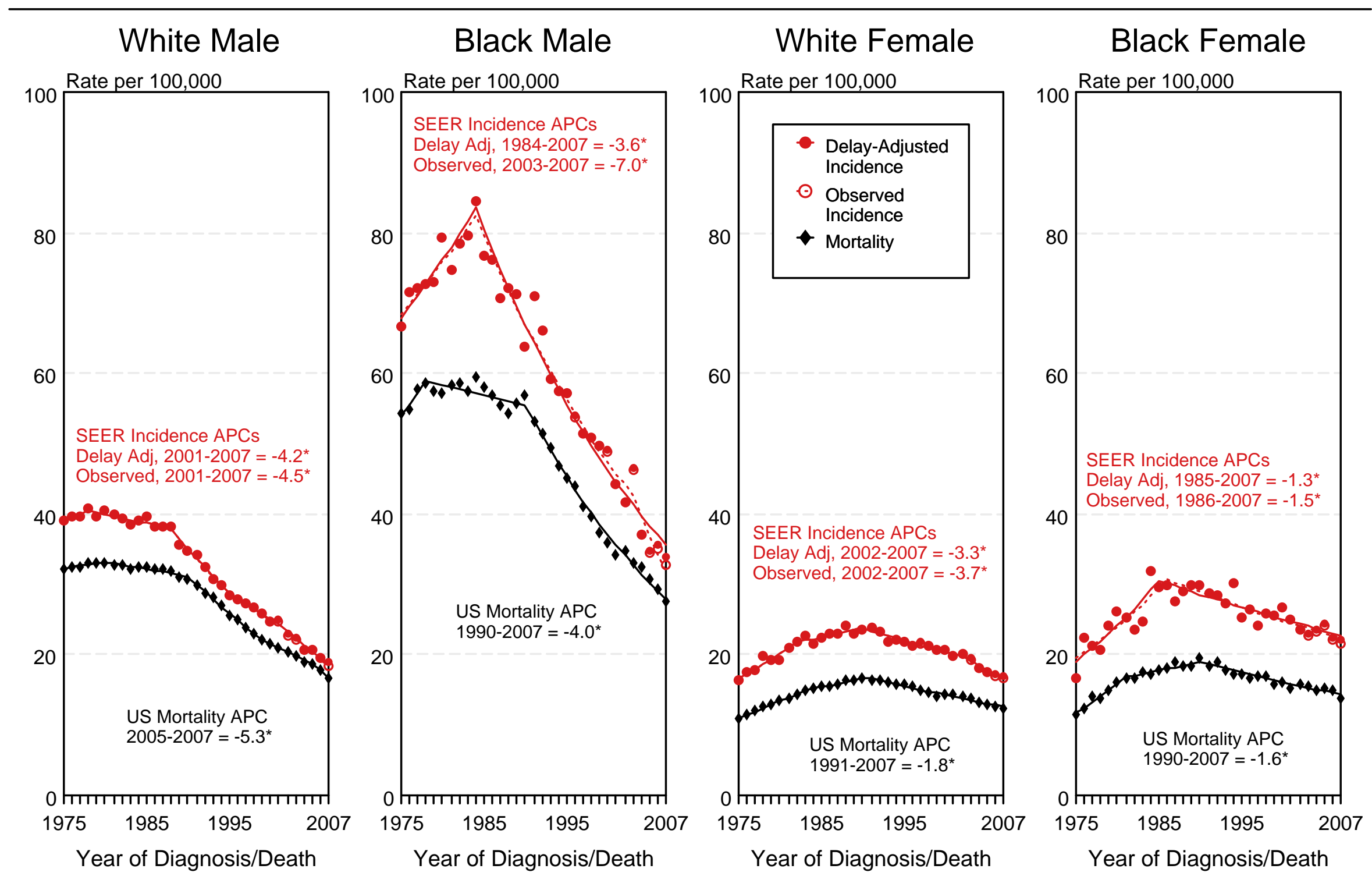

a Source: SEER 9 areas and US Mortality Files (National Center for Health Statistics, CDC).

Rates are age-adjusted to the 2000 US Std Population (19 age groups - Census P25-1103).

Regression lines and APCs are calculated using the Joinpoint Regression Program Version 3.4.3, April 2010, National Cancer Institute.

The APC is the Annual Percent Change for the regression line segments. The APC shown on the graph is for the most recent trend.

* The APC is significantly different from zero $(p<0.05)$. 


\section{SEER Observed Incidence, SEER Delay Adjusted Incidence and US Death Rates ${ }^{a}$ Cancer of the Lung and Bronchus, Ages 65 and Over, by Race and Sex}

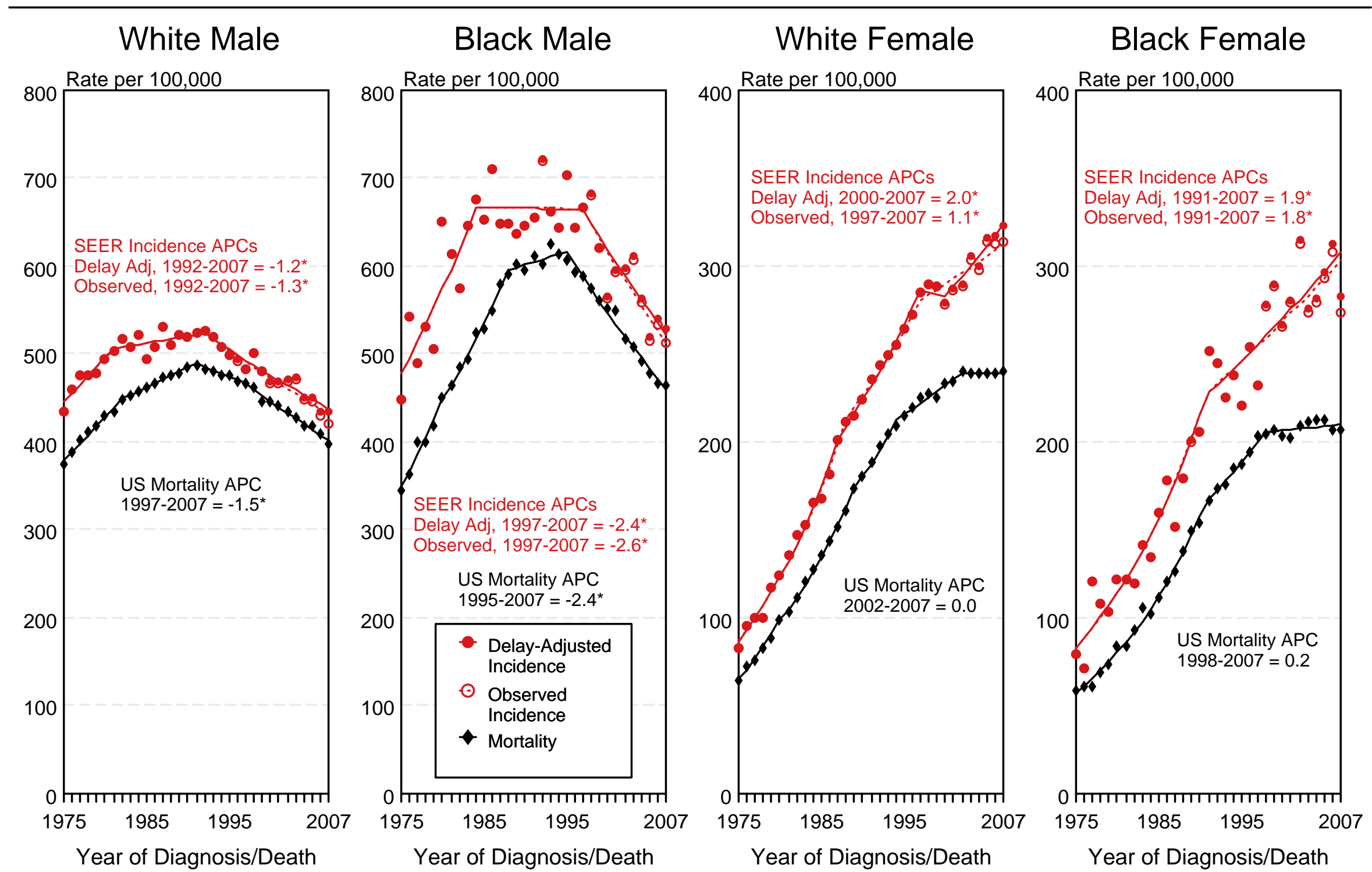

a Source: SEER 9 areas and US Mortality Files (National Center for Health Statistics, CDC).

Rates are age-adjusted to the 2000 US Std Population (19 age groups - Census P25-1103).

Regression lines and APCs are calculated using the Joinpoint Regression Program Version 3.4.3, April 2010, National Cancer Institute.

The APC is the Annual Percent Change for the regression line segments. The APC shown on the graph is for the most recent trend.

* The APC is significantly different from zero $(p<0.05)$. 


\section{SEER Incidence and US Death Rates ${ }^{a}$ \\ Cancer of the Lung and Bronchus, Both Sexes \\ Joinpoint Analyses for Whites and Blacks from 1975-2007}

and for Asian/Pacific Islanders, American Indians/Alaska Natives and Hispanics from 1992-2007

\section{Incidence}

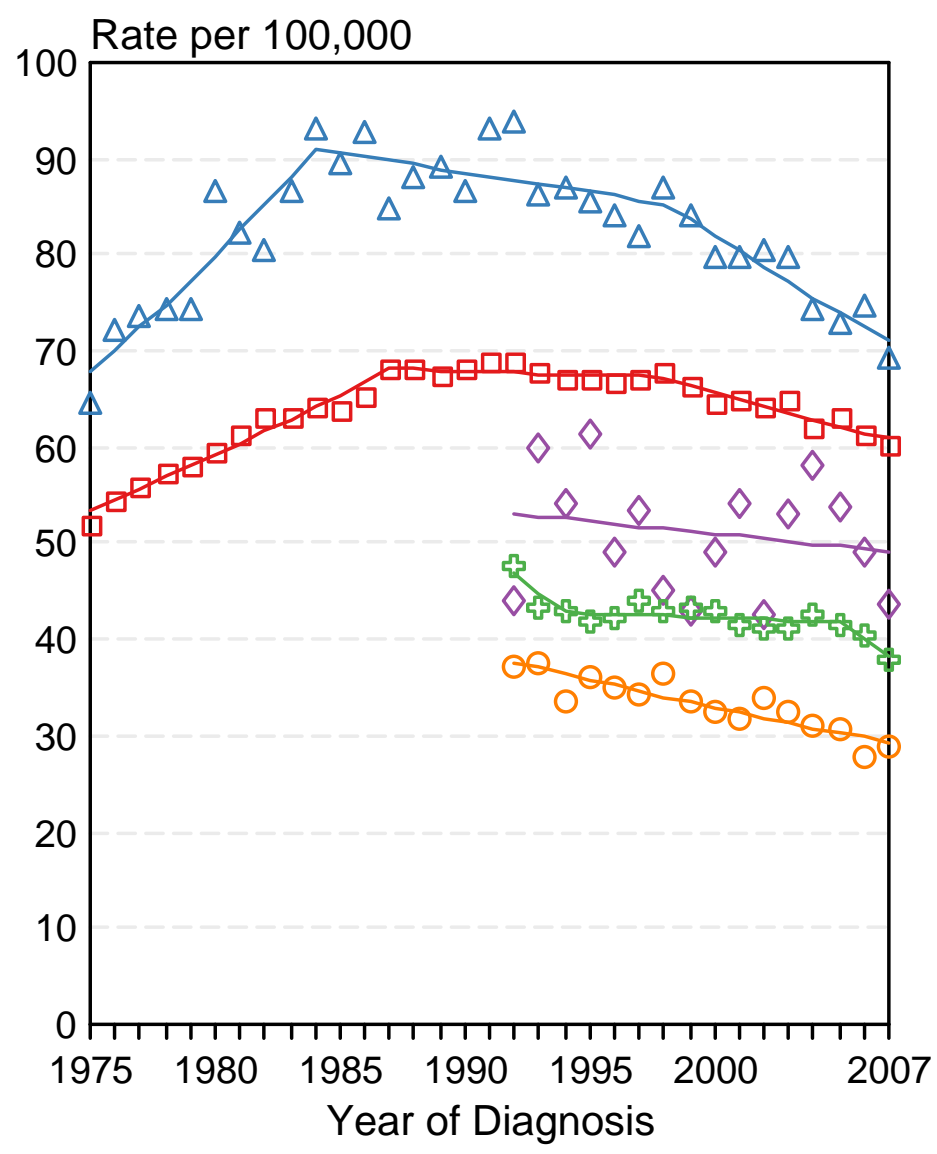

Mortality
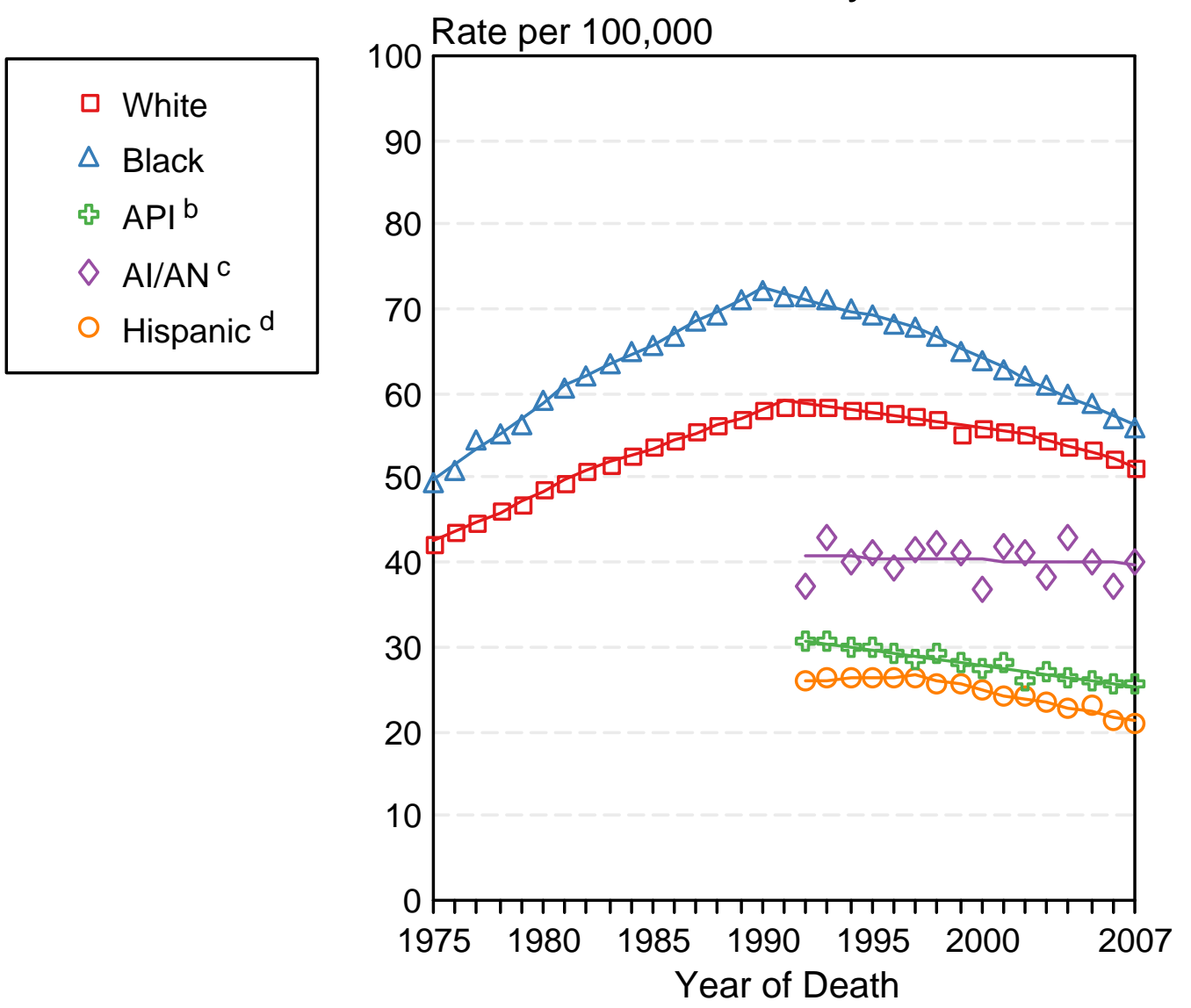

Source: Incidence data for whites and blacks are from the SEER 9 areas (San Francisco, Connecticut, Detroit, Hawaii, lowa, New Mexico, Seattle, Utah, Atlanta).

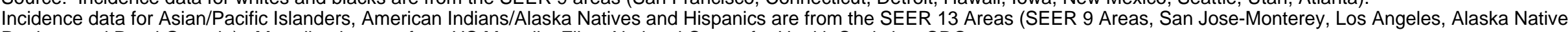
Registry and Rural Georgia). Mortality data are from US Mortality Files, National Center for Health Statistics, CDC.

a Rates are age-adjusted to the 2000 US Std Population (19 age groups - Census P25-1103).

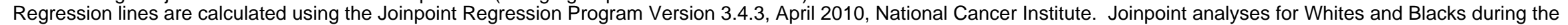
1975-2007 period allow a maximum of 4 joinpoints. Analyses for other ethnic groups during the period 1992-2007 allow a maximum of 2 joinpoints.

b $\mathrm{API}=$ Asian/Pacific Islander.

c $\mathrm{Al} / \mathrm{AN}=$ American Indian/Alaska Native. Rates for American Indian/Alaska Native are based on the CHSDA(Contract Health Service Delivery Area) counties.

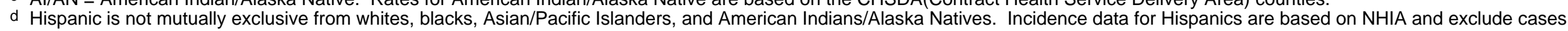
from the Alaska Native Registry. Mortality data for Hispanics exclude cases from Connecticut, the District of Columbia, Maine, Maryland, Minnesota, New Hampshire, New York, North Dakota, Oklahoma, and Vermont. 


\section{Cancer of the Lung \& Bronchus SEER Delay-Adjusted Incidence \& US Death Rates, 1975-2007, All Races, By Sex}

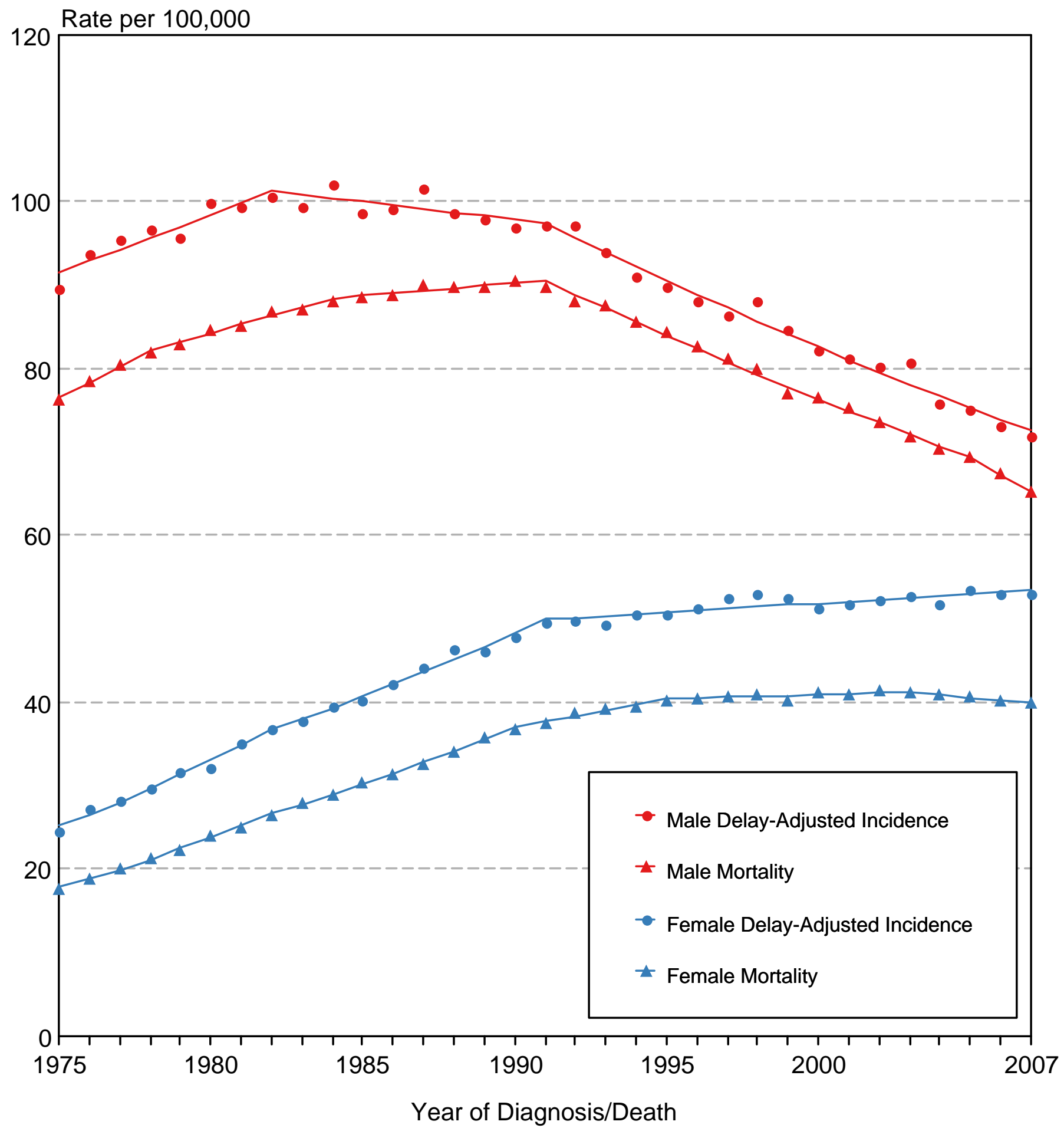

Source: SEER 9 areas and US Mortality Files (National Center for Health Statistics, CDC).

Rates are age-adjusted to the 2000 US Std Population (19 age groups - Census P25-1103).

Regression lines are calculated using the Joinpoint Regression Program Version 3.4.3, April 2010, National Cancer Institute. 


\section{Cancer of the Lung \& Bronchus SEER Delay-Adjusted Incidence, 1975-2007 By Sex and Race}

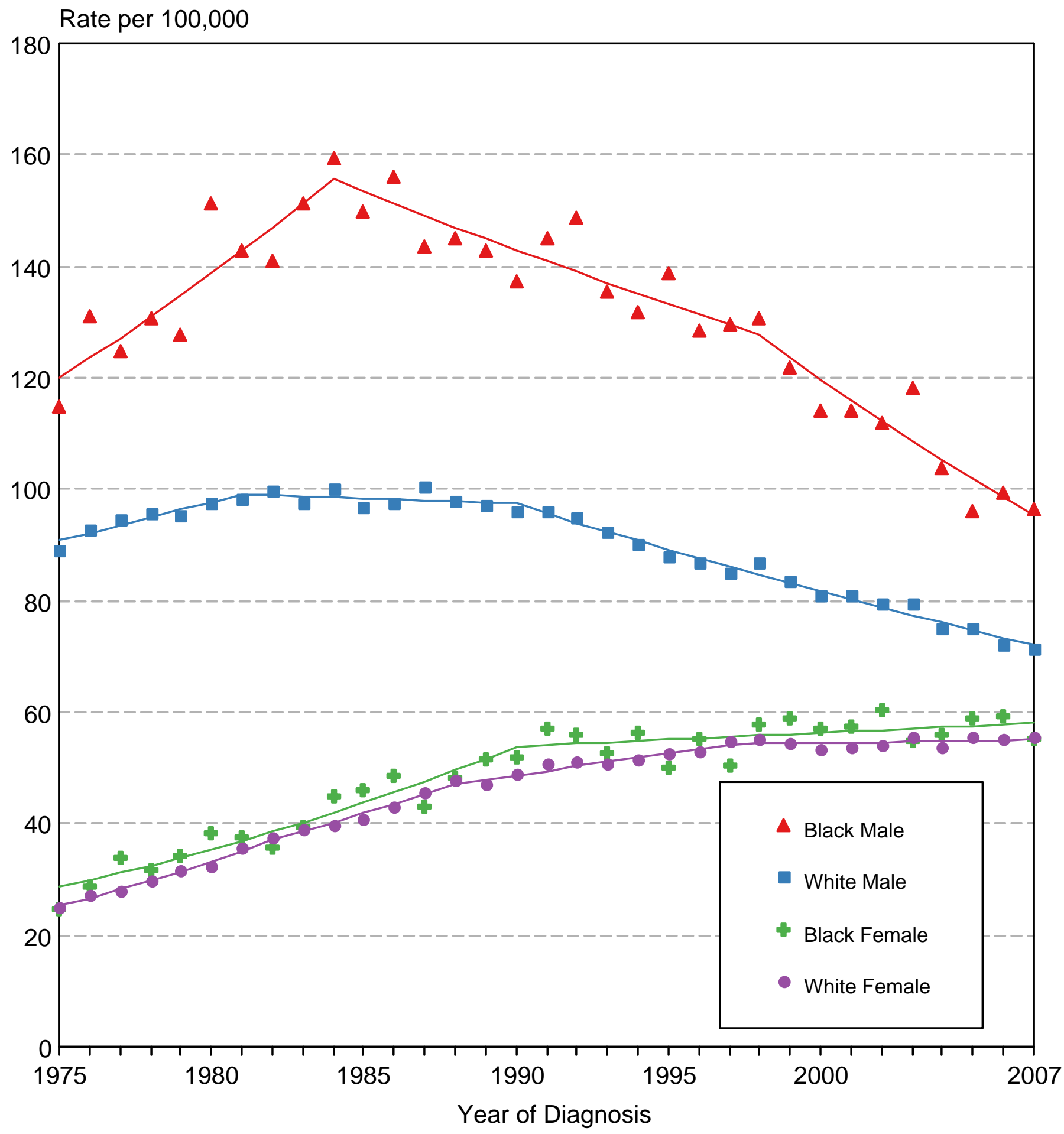

Source: SEER 9 areas. Rates are age-adjusted to the 2000 US Std Population (19 age groups - Census P25-1103). Regression lines are calculated using the Joinpoint Regression Program Version 3.4.3, April 2010, National Cancer Institute. 


\section{SEER Observed Incidence and SEER Delay Adjusted Incidence Rates ${ }^{a}$ SEER 9 Areas Compared to SEER 13 Areas Cancer of the Lung and Bronchus, Male, by Race}

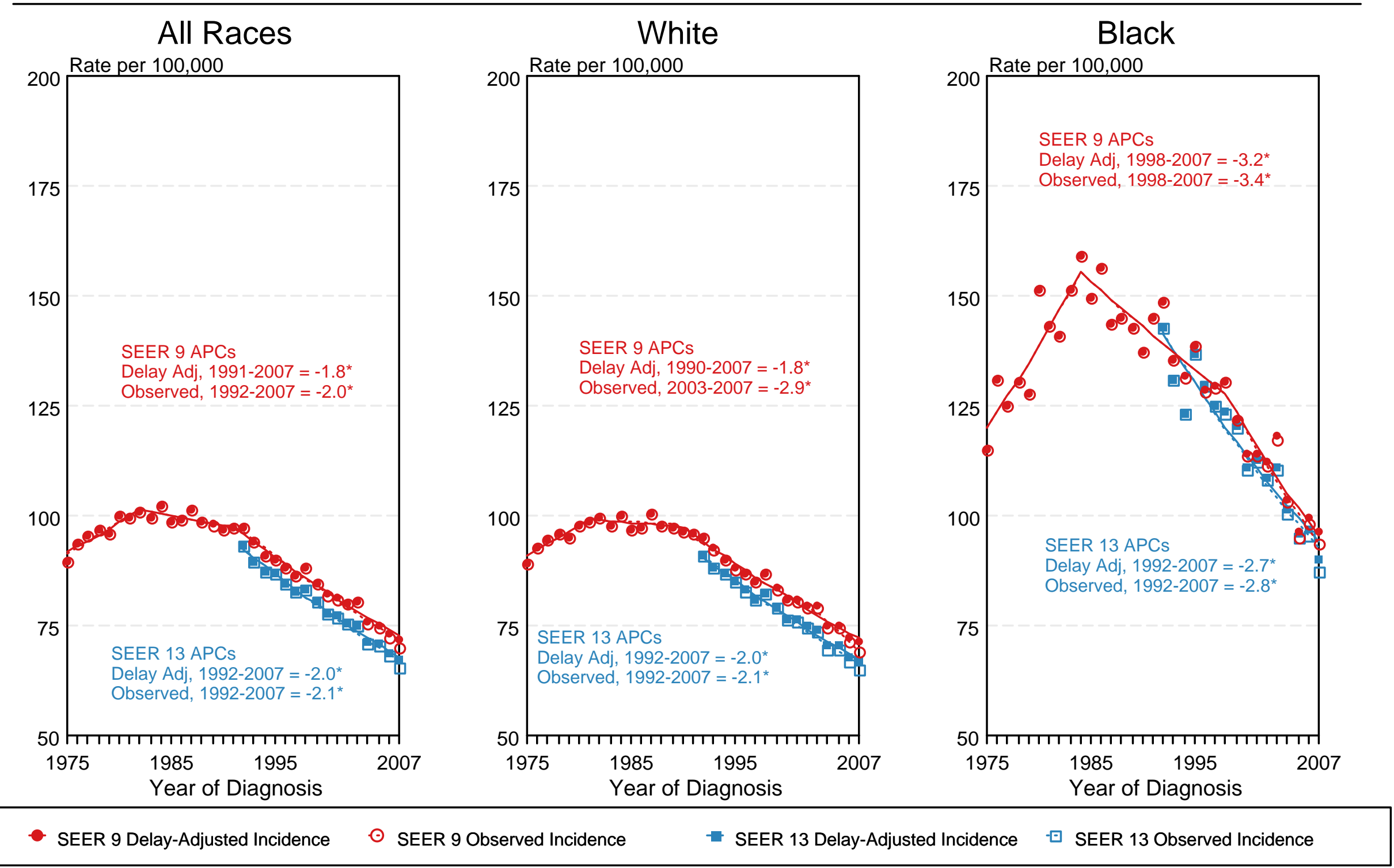

a Source: SEER 9 areas and SEER 13 areas.

Rates are age-adjusted to the 2000 US Std Population (19 age groups - Census P25-1103).

Regression lines and APCs are calculated using the Joinpoint Regression Program Version 3.4.3, April 2010, National Cancer Institute.

The APC is the Annual Percent Change for the regression line segments. The APC shown on the graph is for the most recent trend.

* The APC is significantly different from zero $(p<0.05)$. 


\section{SEER Observed Incidence and SEER Delay Adjusted Incidence Rates ${ }^{a}$ SEER 9 Areas Compared to SEER 13 Areas Cancer of the Lung and Bronchus, Female, by Race}

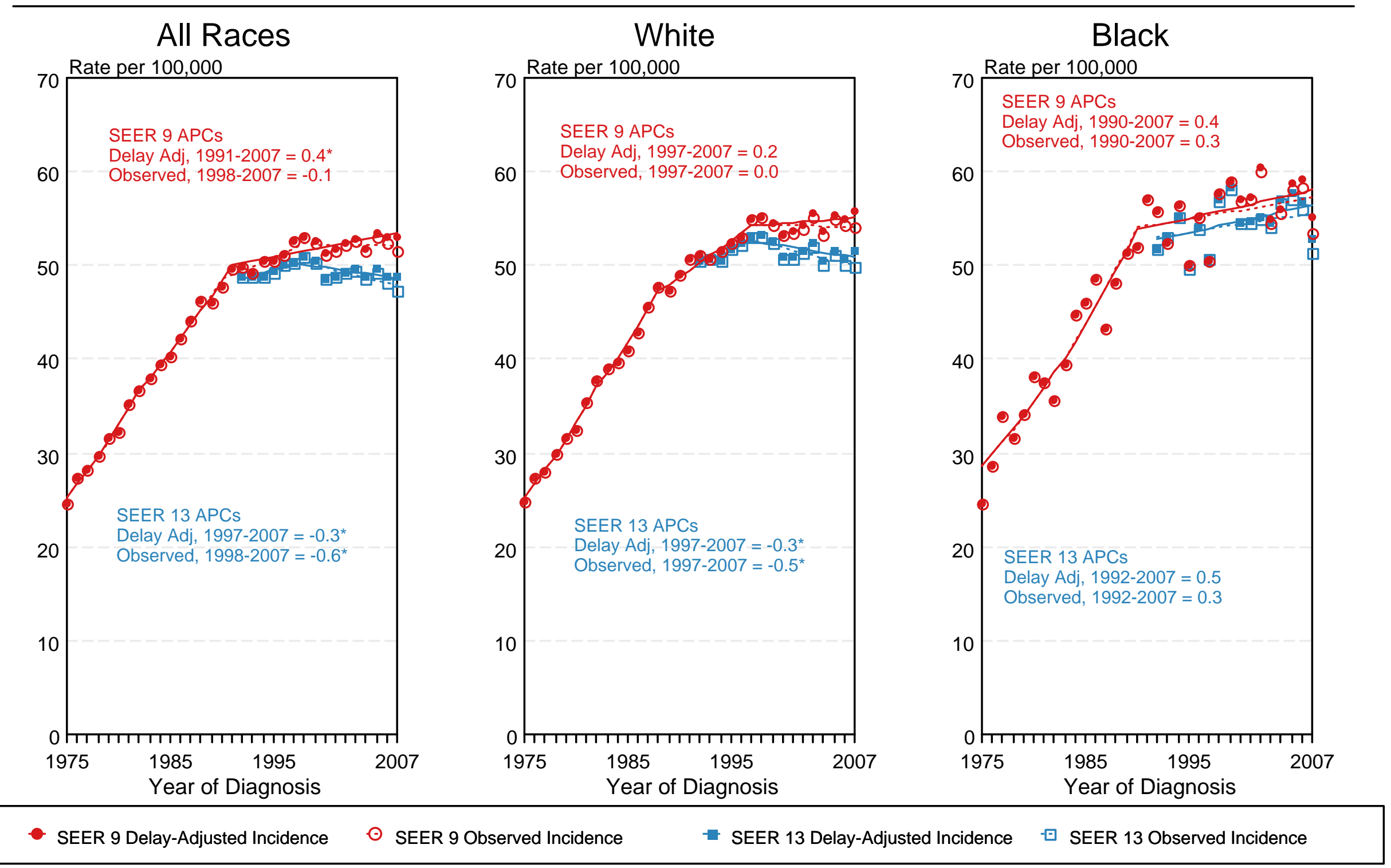

a Source: SEER 9 areas and SEER 13 areas.

Rates are age-adjusted to the 2000 US Std Population (19 age groups - Census P25-1103).

Regression lines and APCs are calculated using the Joinpoint Regression Program Version 3.4.3, April 2010, National Cancer Institute.

The APC is the Annual Percent Change for the regression line segments. The APC shown on the graph is for the most recent trend.

* The APC is significantly different from zero $(p<0.05)$. 


\section{Cancer of the Lung and Bronchus \\ 5-Year SEER Conditional Relative Survival and 95\% Confidence Intervals \\ Probabilty of surviving the next 5 years given the cohort \\ has already survived 0,1 , or 3 years \\ $1998-2006$ by stage at diagnosis}
Survival Time Since Diagnosis
0 year (at diagnosis)
1 year
3 years

Percent Surviving Next 5 Years

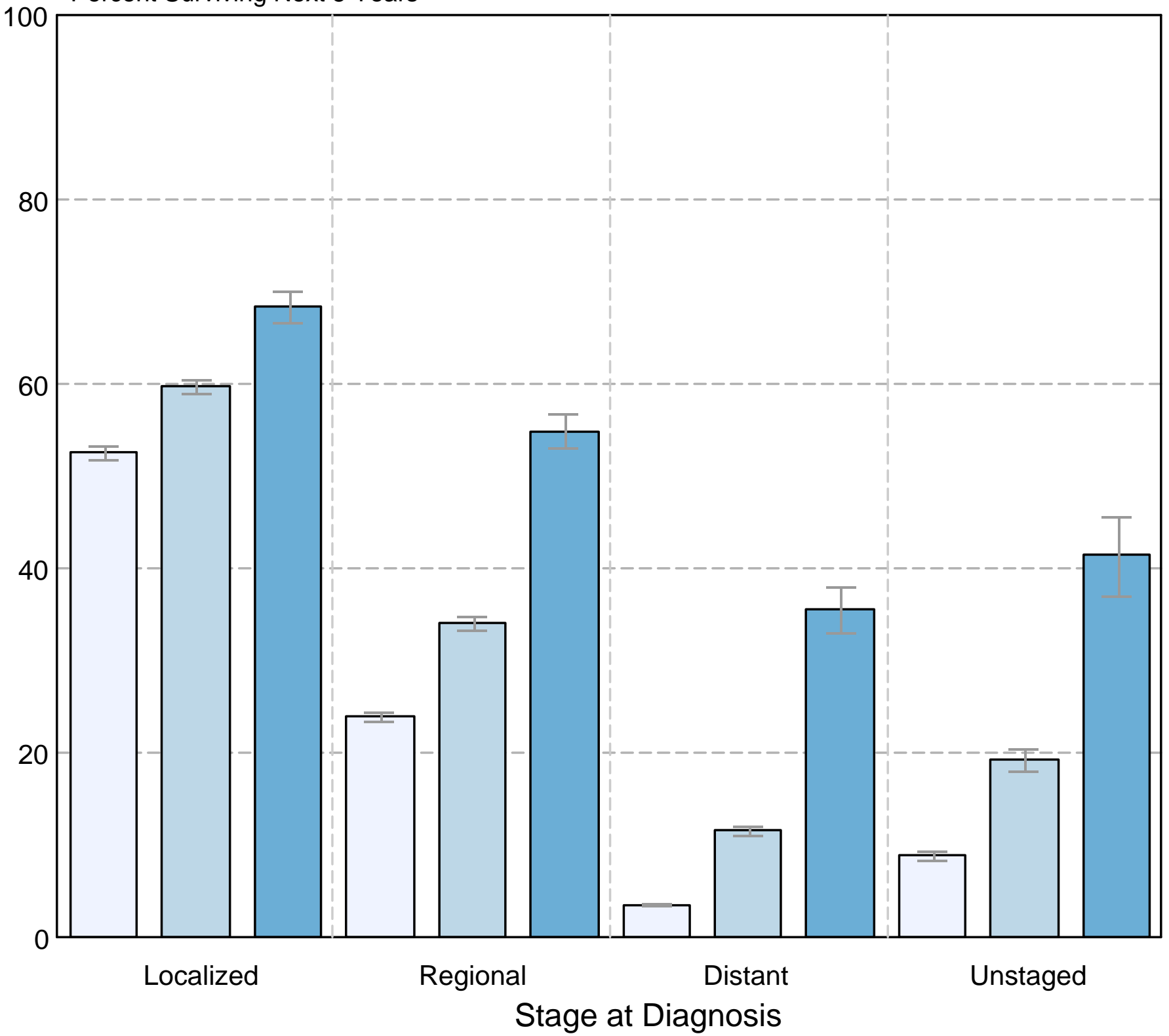

Source: SEER 17 areas (San Francisco, Connecticut, Detroit, Hawaii, lowa, New Mexico, Seattle, Utah, Atlanta, San Jose-Monterey, Los Angeles, Alaska Native Registry, Rural Georgia, California excluding SF/SJM/LA, Kentucky, Louisiana, and New Jersey). California excluding SF/SJM/LA, Kentucky, Louisiana, and New Jersey contribute cases for diagnosis years 2000-2006. The remaining 13 SEER Areas contribute cases for the entire period 1998-2006.

Percent surviving is not shown if based on less than 25 cases.

Confidence intervals are not shown if length of the confidence interval is greater than 5 times the standard error. 